\title{
Hypercohomology Spectra and Thomason's Descent Theorem
}

\author{
Stephen A. Mitchell* \\ Revised October 1996
}

Dedicated to the memory of Bob Thomason

The celebrated Lichtenbaum-Quillen conjectures predict that for a sufficiently nice scheme and given prime $\ell$, the $\ell$-adic algebraic K-groups of $\mathrm{X}$ are closely related to the $\ell$-adic étale cohomology groups of X. More precisely, one version of the conjectures asserts that there is a descent spectral sequence of Atiyah-Hirzebruch type

$$
H_{e ́ t}^{p}\left(X ; Z_{\ell}\left(\frac{q}{2}\right)\right) \Rightarrow \pi_{q-p}\left(K X_{\ell}^{\wedge}\right)
$$

but with the convergence only valid in sufficiently high degrees. Here the coefficient sheaves are Tate twists of the $\ell$-adic integers, and are to be interpreted as zero if $q$ is odd. Throughout this paper, étale cohomology is continuous étale cohomology [19], and the indicated abutment of the spectral sequence consists of the homotopy groups of the Bousfield $\ell$-adic completion of the spectrum KX, not the naive $\ell$-adic completion of the K-groups.

In a remarkable paper [42], Thomason proved the Lichtenbaum-Quillen conjectures for a certain localized form of K-theory - so-called "Bott-periodic" K-theory. The first step was the development of an elaborate theory of hypercohomology spectra $\mathbb{H}^{\cdot}(X ; \mathcal{E})$ associated to étale presheaves of spectra $\mathcal{E}$ - or more generally, to presheaves of spectra on a Grothendieck site. These hypercohomology spectra are by their very construction naturally

\footnotetext{
*Supported by a grant from the National Science Foundation
} 
equipped with a suitable descent spectral sequence, and there is a natural map $\mathcal{E}(X) \longrightarrow \mathbb{H}^{\cdot}(X ; \mathcal{E})$. In particular, the $\mathrm{K}$-theory of a scheme X maps to its associated hypercohomology spectrum $\mathbb{H}^{*} e ́ t(X ; K)$.

Fix a prime $\ell$, which we will assume is odd in order to simplify the discussion.Let $L(-)$ denote Bousfield localization with respect to complex $\mathrm{K}$-theory, and let $\hat{L}(-)$ denote its $\ell$-adic completion. The main theorem of $[42]$ can be stated as follows.

Theorem 0.1 Let X be a separated noetherian regular scheme of finite Krull dimension, with sufficiently nice residue fields of characteristic different from $\ell$. Then the natural map $K X \longrightarrow \mathbb{H}^{\cdot}{ }^{e}(X ; K)$ induces a weak equivalence

$$
\hat{L} K(X) \stackrel{\cong}{\longrightarrow} \hat{L} \mathbb{H}^{\cdot} e^{\prime}(X ; K)
$$

and there is a convergent descent spectral sequence

$$
H_{e ́ t}^{p}\left(X ; Z_{\ell}\left(\frac{q}{2}\right)\right) \Rightarrow \pi_{q-p} \hat{L} K X
$$

For a precise statement see Theorem 4.13. Several remarks are in order:

- Usually the theorem is stated in terms of Bott-periodic K-theory. There are non-nilpotent elements

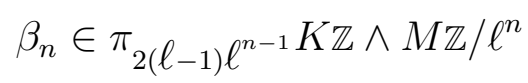

that ultimately arise from a primitive $\ell^{n}$-th root of unity $\left(M \mathbb{Z} / \ell^{n}\right.$ is the $\bmod \ell^{n}$ Moore spectrum). Using the $K \mathbb{Z}$-algebra structure on $K X$, we can form the mapping telescope $\beta_{n}^{-1} K X \wedge M \mathbb{Z} / \ell^{n}$, and it is known that this mapping telescope is weak equivalent to $\hat{L} K X \wedge M \mathbb{Z} / \ell^{n}$. Thus one gets a spectral sequence going from $\bmod \ell^{n}$ cohomology to $\bmod \ell^{n}$ Bott-periodic K-theory. In this paper, however, the emphasis will be on Bousfield localization, with the Bott elements entering only as tools in the proofs. For more information on Bott elements, see [31] and the references cited there, as well as the recent paper by B. Kahn [24].

- The localization functor $\hat{L}$ is only the first of an infinite sequence of localization functors $\hat{L}_{n}$ defined on spectra. Thus from a global 
homotopy-theoretic perspective, the localization imposed by Thomason is a priori rather drastic. However the author showed in [30] that Theorem 0.1 remains true with $\hat{L}$ replaced by $\hat{L}_{n}$ for any $n$, including the "harmonic localization" $\hat{L}_{\infty}$, so this is not such a drastic localization after all. For further discussion of this point, see [31].

- The second statement of the theorem is not merely a corollary of the first. The difficulty is with the identification of the $E_{2}$-term; nowadays this depends on the Gabber rigidity theorem for hensel local rings and Suslin's theorem on algebraically closed fields. When Thomason first proved Theorem 0.1 , however, these results were not available and he had to prove them in localized form.

- As a corollary to another major work [44], Thomason showed that if K-theory is replaced by Bass K-theory, the assumption that $\mathrm{X}$ is separated and regular can be dropped. This more general result will not be considered here.

Thomason's theorem has many interesting applications. For example, let $\mathrm{F}$ be a totally real number field with ring of integers $\mathcal{O}_{F}$. Let $L K_{n}(-)=$ $\pi_{n} L K(-)$. Then up to powers of 2 the value of the Dedekind zeta function at the odd negative integers is given by the amazing formula

$$
\zeta_{F}(1-2 n)=\frac{\left|L K_{4 n-2} \mathcal{O}_{F}\right|}{\left|L K_{4 n-1} \mathcal{O}_{F}\right|}
$$

The point is that the descent spectral sequence collapses, and the theorem of Wiles [46] proving Iwasawa's "Main Conjecture" computes the given zeta values in terms of étale cohomology.

Another application, due to Bill Dwyer and the author [9], uses Thomason's theorem to compute the $\ell$-adic complex K-theory of the spectrum $K \mathcal{O}_{F}\left[\frac{1}{\ell}\right]$ in terms of the Iwasawa theory of the cyclotomic $Z_{\ell^{-}}$extension ( $\mathrm{F}$ an arbitary number field). In the case of abelian fields, this leads to a computation of the complex K-theory of the space $B G L \mathcal{O}_{F}\left[\frac{1}{\ell}\right]^{+}$. This work also yields an explicit conjectural description of the Hopf algebra $H_{*}\left(B G L \mathcal{O}_{F}\left[\frac{1}{\ell}\right]^{+} ; \mathbb{Z} / \ell\right)$, and leads to some partial computations in [32] and [33].

Further applications and motivation can be found in [42], [43], and [31]. The purpose of the present paper, however, is to explain the proof of Thomason's Theorem, and the vast array of machinery underlying it. It took the 
author rather a long time to progress from "thrill seeker" to "reckless cheat" to "honest man" (Thomason's amusing terminology; see [42], introduction). Our hope is that this essay will smooth the way for others. At the same time, we will survey the work of Jardine [20] [21] [22] that recasts the entire theory in terms of closed model categories. In this approach, the role of the hypercohomology presheaf $\mathbb{H}^{\cdot}(-; \mathcal{E})$ is played by a globally fibrant model for $\mathcal{E}$. Here again, the technical prerequisites are formidable; our goal is to help make these works more accessible.

The paper is organized as follows. Each of the five sections has its own introduction. The first two sections are intended for readers who may not be familiar with schemes and sites. Section 1 is an introduction to Thomason's theorem for fields, eschewing all scheme-theoretic language to minimize the prerequisites. Section 2 provides a short introduction to sites and cohomology, and includes a discussion of the very useful Nisnevich site.

The experts can proceed directly to the third section. Here we introduce presheaves of spectra on a Grothendieck site, and the associated hypercohomology spectrum. The latter construction is a direct generalization to spectra of the Godement resolution of an abelian sheaf. Then we consider Jardine's closed model category structure for presheaves of spectra on a site.

Section 4 begins by discussing the descent theorems of Brown-Gersten and Nisnevich. Then the Nisnevich descent theorem is used to deduce Theorem 0.1 for schemes from Theorem 0.1 for fields. Section 5 sketches the ingenious proof for fields.

Acknowledgements: I would especially like to thank Rick Jardine for conversations and correspondence, particularly concerning his book [22], on which much of this paper is based. Thanks are also due to Paul Goerss, for sharing his expertise on closed model categories and related matters, and to Bill Dwyer for helpful conversations on the material of Section 5.

Notation, terminology, and a confession of sins. The mod $n$ Moore spectrum is denoted $M \mathbb{Z} / n$. For a spectrum $\mathcal{E}$, the $\ell$-adic completion (=Bousfield localization with respect to $M \mathbb{Z} / \ell$ ) is denoted $\mathcal{E}^{\wedge}$. We write $L \mathcal{E}$ for Bousfield localization with respect to complex K-theory, and abbreviate $(L \mathcal{E})^{\wedge}$ as $\hat{L} \mathcal{E}$. Recall also that $\hat{L}(-)$ is the same thing as Bousfield localization with respect to mod $\ell$ complex K-theory. Bear in mind that $\mathrm{L}$ commutes with pseudo-filtered (e.g. direct) colimits, but $\hat{L}$ does not. Readers unfamiliar 
with Bousfield localization might consult [31].

If $\mathrm{S}$ is a topological space, possibly discrete, $\operatorname{Map}(S, X)$ denotes the space (spectrum) of pointed maps to the pointed space (spectrum) X.

If $\mathrm{A}$ is a presheaf of sets or abelian groups, we usually write $a A$ for the sheafification of $\mathrm{A}$, but when $A=\pi_{q} \mathcal{E}$ is a presheaf of homotopy groups attached to a presheaf of spectra, we follow a common practice and write $\tilde{\pi}_{q} \mathcal{E}$ for $a \pi_{q} \mathcal{E}$.

The K-theory spectrum of the category of locally free sheaves on a scheme $\mathrm{X}$ is denoted KX. The K-theory spectrum attached to the coherent sheaves is denoted $K^{\prime} X$, since the alternate notation $G X$ would just about exhaust the already overworked letter $\mathrm{G}$.

Now, as to our sins: Among the many technical problems that arise in the theory discussed here, there are several that we have chosen to ignore. Let us at least be explicit about what they are. First, there are the usual set-theoretic difficulties - for example, many of our categorical definitions only make sense if the category in question is small. Occasionally hypotheses of this sort are made explicitly, but more often it is left to the reader to make the necessary adjustments. Second, there is the problem that some of the "functors" lurking in the background are in fact only pseudo-functors for example, the "functor" that assigns to each ring its category of finitelygenerated projective modules. In addition, the passage from schemes to K-theory spectra is left inside a black box - particularly when it comes to questions involving the multiplicative structure. For a careful and rigorous treatment of all these matters, see [22].

\section{Contents}

1. Thomason's theorem for fields. Homotopy fixed point sets. Galois cohomology. A provisional definition of hypercohomology spectra for fields. Statement of Thomason's descent theorem for fields.

2. Schemes, sites and cohomology. Schemes, étale morphisms, and hensel local rings. Grothendieck sites: the étale, Nisnevich and Zariski sites. Presheaves and sheaves. Points and stalks. Sheaf cohomology and cohomological dimension. Čech cohomology. Noetherian sites. 
3. Hypercohomology spectra. Spectral sequences, closed model categories, filtered colimits. Hypercohomology: Thomason's Godement construction for spectra. Jardine's closed model category structure for presheaves of spectra. $\check{C}$ ech hypercohomology spectra.

4. Descent theorems in K-theory. The Brown-Gersten theorem (Zariski descent for K-theory). Nisnevich descent. Gabber rigidity and the K-theory sheaf on the étale site. Thomason's descent theorem and the proof for schemes.

5. The proof of Thomason's theorem for fields. Hyperhomology spectra. Norm and hypernorm. The analogue for spectra of a theorem of Tate. Transfer and hypertransfer. The proof, at last.

\section{Thomason's Theorem for Fields}

Let $\mathrm{F}$ be a field, $\ell$ a prime not equal to the characteristic of $\mathrm{F}$. One of the fundamental problems of algebraic K-theory is to compute the $\ell$-adic Ktheory of $\mathrm{F}$, where by " $\ell$-adic K-theory" we mean the homotopy groups of the $\ell$-adic completion $K F^{\wedge}$. The following beautiful theorem was proved by Suslin in 1984 [38] [39].

Theorem 1.1 Let $F$ be a separably closed field, $\ell$ a prime not equal to the characteristic of F. Then

$$
\pi_{2 n} K F^{\wedge} \cong Z_{\ell}(n)
$$

for $n \geq 0$, and

$\pi_{\text {odd }} K F^{\wedge}=0$. In fact there is a weak equivalence $K F^{\wedge} \cong$ bu^, where bu is the -1-connected cover of the complex K-theory spectrum.

Here $Z_{\ell}(\mathrm{n})$ denotes the $\mathrm{n}$-th Tate twist of the $\ell$-adic integers. In other words,

$$
Z_{\ell}(n)=\otimes^{n} Z_{\ell}(1)
$$

where $Z_{\ell}(1)$ denotes the inverse limit of the groups $\mu_{\ell^{\nu}}(F)$. 
Theorem 1.1 was originally stated for algebraically closed fields, but the extension to the separably closed case is immediate because $\ell$-adic K-theory (with $\ell \neq$ char $F$ ) is invariant under purely inseparable extension $([35], 7,4.8$ ). We also note that there is some choice involved in the weak equivalence $K F^{\wedge} \cong b u^{\wedge}$. It is defined by a diagram

$$
K F^{\wedge} \longleftarrow K E^{\wedge} \longrightarrow b u^{\wedge}
$$

Here $\mathrm{E}$ is the algebraic closure of the prime subfield, and the first map is just the canonical inclusion. This map is a weak equivalence [38]. If Char $\mathrm{F}=0$, the second map arises from a choice of embedding of E into the complex numbers. If Char $\mathrm{F}=\mathrm{p}$, it arises in effect from a choice of Brauer lift (or equivalently, by a choice of embedding of the Witt vectors of $\mathrm{E}$ into $\mathbb{C}$ ).

Given Suslin's theorem, it is natural to try to compute the K-theory of F by "descent" from its separable closure. In other words, one wants some kind of spectral sequence whose input is $\pi_{*} K \bar{F}^{\wedge}$ as Galois module and whose output is $\pi_{*} K F^{\wedge}$. The way to approach such a problem is to cook up an auxiliary spectrum $\mathrm{Y}$ which by its very construction admits such a spectral sequence, and which is equipped with a natural map $K F^{\wedge} \rightarrow Y$. Then one tries to show this map is a weak equivalence. In fact, however, life is not so simple; it turns out that the best one can hope for is a weak equivalence on some connected cover. All this will be discussed further once we have Thomason's descent machinery in place. We will first try to motivate this machinery via the prototypical example of homotopy fixed point sets.

\subsection{Homotopy fixed point sets}

Let $\mathrm{G}$ be a discrete group, $\mathrm{X}$ a $\mathrm{G}$-spectrum. Here we simply mean that each space $X_{n}$ of the spectrum is a pointed G-space, and the structure maps $X_{n} \longrightarrow \Omega X_{n+1}$ are G-maps (with $\mathrm{G}$ acting trivially on the loop coordinate). The homotopy fixed-point spectrum is

$$
X^{h G}=\operatorname{Map}^{G}\left(E G_{+}, X\right)
$$

Here the n-th space of the spectrum on the right is the function space of equivariant pointed maps $\operatorname{Map}^{G}\left(E G_{+}, X_{n}\right)$. The structure maps are the usual, obvious ones. Filtering EG by skeleta exhibits $X^{h G}$ as the inverse limit of a tower of fibrations 


$$
\ldots \longleftarrow \operatorname{Map}^{G}\left((E G)_{+}^{n-1}, X\right) \longleftarrow \operatorname{Map}^{G}\left((E G)_{+}^{n}, X\right) \longleftarrow \ldots
$$

in which the fibres have the form $\prod \Omega^{n} X$. This yields a "conditionally convergent" right half-plane cohomology spectral sequence - the "descent spectral sequence"

$$
E_{2}^{p,-q}=H^{p}\left(G ; \pi_{q} X\right) \Longrightarrow \pi_{q-p} X
$$

See section 3.1 for a discussion of convergence. Here it will suffice to note that any of the following conditions will guarantee convergence:

- $\mathrm{G}$ has finite cohomological dimension - or more generally, there exists a positive integer $d$ such that $H^{p}\left(G ; \pi_{q} X\right)=0$ for all $p>d$ and all $q$. In this case the spectral sequence collapses at $E_{d+1}$.

- The spectrum $X$ is bounded above - that is, there exists an integer $\mathrm{M}$ such that $\pi_{q} X=0$ for all $q>M$. In this case the spectral sequence is formally the same as a first quadrant spectral sequence.

- The groups $H^{p}\left(G ; \pi_{q} X\right)$ are all finite.

There is a natural map $X^{G} \longrightarrow X^{h G}$ defined as the composite

$$
X^{G}=\operatorname{Map}^{G}\left(S^{0}, X\right) \longrightarrow \operatorname{Map}^{G}\left(E G_{+}, X\right)
$$

induced by the augmentation $E G_{+} \longrightarrow S^{0}$. If this map is a weak equivalence, then the descent spectral sequence converges to the homotopy of the actual fixed point set.

There is another way to describe $X^{h G}$ that will be essential later. Recall that $E G_{+}$is the geometric realization of a certain simplicial pointed G-set $E . G_{+}$whose n-th level is $G_{+}^{n+1}$. Thinking of $E . G_{+}$as a simplicial pointed discrete space, we can apply the functor $\operatorname{Map}^{G}(-, X)$ to obtain a cosimplicial spectrum that we will denote $S \cdot(G, X)$. Then

$$
X^{h G}=\operatorname{Tot} S(G, X)
$$

where "Tot" is the Bousfield-Kan total space construction [6] applied levelwise. It is shown in [6] that this Tot is the inverse limit of a tower of fibrations 
- and indeed it is just the same tower constructed above. Moreover there is a weak equivalence

$$
X^{h G} \cong \operatorname{holim}_{\Delta} S \cdot(G, X)
$$

and it is this holim construction that we will actually use.

Roughly speaking, we want to apply this machinery to the case $X=K \bar{F}$, where $\bar{F}$ is the separable closure of F, with $G=G_{F}$ the absolute Galois group. However we need to modify the homotopy fixed point construction in two ways. First, $\mathrm{G}$ is a profinite group and we want to take into account this profinite structure. In particular we want a spectral sequence involving Galois cohomology. ${ }^{1}$ Secondly, we will change our point of view by replacing the $G_{F}$-spectrum $K \bar{F}$ by the "presheaf of spectra" $L \mapsto K L$, L a finite separable extension of $\mathrm{F}$ (for an elegant treatment of homotopy fixed point spaces for Galois groups, see [15]). We first digress to discuss Galois cohomology.

\subsection{Galois cohomology}

Let $\mathrm{G}$ be a profinite group. ${ }^{2}$ This is equivalent to saying that $\mathrm{G}$ is a compact and totally disconnected topological group, but in more practical terms it means that $\mathrm{G}$ is the inverse limit of its finite quotients $G / U$, with $\mathrm{U}$ varying over the closed normal subgroups of finite index (note that the closed subgroups of finite index are precisely the open subgroups). A discrete G-module $\mathrm{M}$ is a $\mathrm{G}$-module such that $\mathrm{M}$ is the union of the various fixed submodules $M^{U}$. The cohomology groups $H^{*}(G ; M)$ can be defined in two equivalent ways.

- "Sheaf": The category of discrete G-modules is abelian and has enough injectives. The functors $H^{*}(G ; M)$ are the derived functors of the left exact functor $M \rightarrow M^{G}$.

\footnotetext{
${ }^{1}$ If $G_{F}$ is finite no modifications are needed. However fields with nontrivial but finite absolute Galois group are extremely rare - the example of the real numbers notwithstanding! See [3],X, Theorem 2.1 for a discussion of this claim.

${ }^{2}$ Logically, the cohomology to be discussed here should be called "profinite group cohomology". However it turns out that every profinite group is the Galois group of some Galois extension [45], so the theories of Galois extensions and profinite groups are abstractly equivalent.
} 
- "Cech": $H^{*}(G ; M)=\operatorname{colim}_{U} H^{*}\left(G / U ; M^{U}\right)$. Here the terms in the colimit are just ordinary group cohomology. The maps in the colimit combine the inclusions $M^{U} \subset M^{V}$ with pullback along the quotients $G / V \rightarrow G / U$ in the obvious way.

The reason for the slogans "sheaf" and "Cech" may not be apparent, but should become clear in the next section.

Here is one way to see that the two definitions agree. We include this here because it helps to illuminate some of the spectrum-level constructions that come later:

Form the simplicial G-set E.G as usual. In this case it is a simplicial space with the profinite topology on the terms $G^{n+1}$. Apply the functor $\operatorname{Map}(-, M)$ - continuous M-valued functions - to obtain a cosimplicial Gmodule $\operatorname{Map}(E . G, M)$. Here the G-action is the usual left action whose fixed points yield the group of equivariant maps. The natural augmentation $M \rightarrow \operatorname{Map}(G, M)$ makes the associated cochain complex into a resolution of $\mathrm{M}$ by acyclic G-modules. Applying the G-invariants functor then yields a cosimplicial abelian group $T^{\cdot}(G, M)$. Of course, this is nothing but the usual bar-type complex (a.k.a. the Godement complex, cf. 2.6). The associated cohomology groups are then precisely the groups $H^{*}(G ; M)$ according to the "sheaf" definition.

On the other hand, continuity in this context just means that

$$
\operatorname{Map}\left(G^{n}, M\right)=\operatorname{colim}_{U} \operatorname{Map}\left((G / U)^{n}, M\right)
$$

It follows easily that

$$
T \cdot(G, M)=\operatorname{colim}_{U} T \cdot\left(G / U, M^{U}\right)
$$

so this is the same as "Cech" cohomology.

There is yet another description that will be useful (compare section 3.1).

Proposition 1.2 For a discrete G-module $M, H^{p}(G, M)=\lim _{\Delta}^{p} T^{\cdot}(G, M)$.

We will also need to consider cohomology with coefficients in non-discrete modules such as $Z_{\ell}(\mathrm{n})$. The category of inverse systems $\left\{M_{n}\right\}$ of discrete G-modules is also abelian with enough injectives, and the composite functor $\left\{M_{n}\right\} \mapsto \lim _{n}^{0} M_{n}^{G}$ is left exact. Its derived functors will be denoted 
$H^{*}\left(G ;\left\{M_{n}\right\}\right)$. This is a special case of "continuous" cohomology in the sense of Janssen [19] and will arise in its more general form in 2.7. Often it is written with a subscript viz. $H_{\text {cont }}^{*}$, but we can dispense with this because as far as we are concerned continuous cohomology is the only cohomology. Note that there are short exact sequences

$$
0 \longrightarrow \lim _{n}^{1} H^{p-1}\left(G ; M_{n}\right) \longrightarrow H^{p}\left(G ;\left\{M_{n}\right\}\right) \longrightarrow \lim _{n}^{0} H^{p}\left(G ; M_{n}\right) \longrightarrow 0
$$

If $\mathrm{M}$ is given as an inverse limit of discrete modules - $M=\lim _{n}^{0} M_{n}$ - we will write simply $H^{*}(G ; M)$ for $H^{*}\left(G ;\left\{M_{n}\right\}\right)$. The main case of interest is $Z_{\ell}(n)=\lim _{m}^{0}\left(\mathbb{Z} / \ell^{m}(n)\right)$.

If $L / F$ is a Galois extension, we will usually write $H^{*}(L / F ; M)$ for $H^{*}(G(L / F) ; M)$ and simply $H^{*}(F ; M)$ for $H^{*}(\bar{F} / F ; M)$.

We say that $c d_{\ell} G=d$ if $d$ is maximal such that there exists a discrete $\ell$-torsion module $\mathrm{M}$ with $H^{d}(G ; M) \neq 0$. An argument using "Shapiro's lemma" shows that if $\mathrm{H}$ is any closed subgroup of $\mathrm{G}, c d_{\ell} H \leq c d_{\ell} G$. Translating into field notation, this implies that if $\mathrm{E}$ is any separable algebraic extension of $\mathrm{F}, c d_{\ell} E \leq c d_{\ell} F$.

Some examples:

- If $\mathrm{F}$ is separably closed, obviously $c d_{\ell} \mathrm{F}=0$ for all $\ell$. In fact it is not hard to show that $c d_{\ell} \mathrm{F}=0$ if and only if $\mathrm{F}$ has no finite separable $\ell$ extensions.

- If $\mathrm{F}$ is finite, $c d_{\ell} \mathrm{F}=1$.

- Tsen's theorem: If $\mathrm{F}$ is a function field of dimension one over a separably closed field $k$ (i.e., $\mathrm{F}$ is a finite separable extension of $k(T)$ ), $c d_{\ell} F \leq 1$.

- If $\mathrm{F}$ is a number field and either $\ell$ is odd or $\mathrm{F}$ is pure imaginary, $c d_{\ell} \mathrm{F}=2$. Otherwise $c d_{2} F=\infty$.

Proposition 1.3 If $F$ has transcendence degree $n$ over a subfield $F_{0}$, then $c d_{\ell} F \leq c d_{\ell} F_{0}+n$. (Usually equality holds; see [3],X,2.1.)

The proof involves inducting over a "Tate-Tsen" filtration; compare the proof of Theorem 5.1 below.

One similarly defines cohomological dimension for inverse systems of $\ell$ torsion modules. 


\subsection{Hypercohomology spectra for fields}

A presheaf of spectra on $\mathrm{F}$ is a covariant functor $\mathcal{E}$ from the category of finite separable extensions L of $\mathrm{F}$ (which we assume to be subfields of a fixed separable closure $\bar{F}$ ). Later this definition will be modified and put in its proper context of "presheaves of spectra on the étale site of F", but for now we want to keep things as simple as possible. The main example we have in mind if of course the presheaf $L \mapsto K L$.

The stalk of a presheaf $\mathcal{E}$ is the colimit over finite separable extensions $\mathrm{L}$ of the spectra $\mathcal{E}(\mathrm{L})$. In practice, the functor $\mathcal{E}$ will be defined on infinite extensions as well and will commute with colimits (up to weak equivalence), so we will write $\mathcal{E}_{\bar{F}}$ for the stalk of $\mathcal{E}$. The stalk provides the connection between the presheaf point of view and the $G_{F}$-spectrum point of view: each presheaf has its stalk, and conversely given a $G_{F}$-spectrum $\mathrm{X}$ we get a presheaf $\mathcal{E}$ with $\mathcal{E}(L)=X^{G_{L}}$.

We will give two equivalent versions of the hypercohomology spectrum $\mathbb{H}^{\prime}(\mathrm{F}, \mathcal{E})$. The first is to be taken as the definition. For any profinite group $\mathrm{G}$ and spectrum X, $\operatorname{Map}(G, X)$ will by definition mean $\operatorname{colim}_{U} M a p(G / U, X)$ (where as usual $U$ ranges over open normal subgroups). Passing to equivariant maps, this means that

$$
\operatorname{Map}^{G}(G, X)=\operatorname{colim}_{U} M a p^{G / U}\left(G / U, X^{U}\right)
$$

- "Sheaf": Apply the functor $\operatorname{Map}^{G_{F}}\left(-, \mathcal{E}_{\bar{F}}\right)$ (continuous maps, as always) to $E . G$, obtaining a cosimplicial spectrum $T \cdot(F, \mathcal{E})$. Define

$$
\mathbb{H}^{\prime}(F, \mathcal{E})=\operatorname{holim}_{\Delta} T^{\cdot}(F, \mathcal{E})
$$

- "Cech": Let $S \cdot(F, \mathcal{E})$ denote the cosimplicial spectrum $\operatorname{colim}_{L} S \cdot(G(L / F), \mathcal{E}(L))$. Define

$$
\check{\mathbb{H}}^{\prime}(F, \mathcal{E})=\operatorname{holim}_{\Delta} S \cdot(F, \mathcal{E})
$$

In fact these two spectra are naturally weak equivalent. The point is that the obvious map of cosimplicial spectra

$$
S^{\cdot}(F, \mathcal{E}) \longrightarrow T \cdot(F, \mathcal{E})
$$

is a weak equivalence at each cosimplicial level and hence induces a weak equivalence on holim $_{\Delta}$. 
It is important to note that the two limit operations in the definition of the Cech hypercohomology spectrum do not, in general, commute up to weak equivalence (but see Example 3.22 below).

Theorem 1.4 Let $F$ be any field, $\mathcal{E}$ a presheaf of spectra on $F$. There is a conditionally convergent right half-plane cohomology spectral sequence

$$
E_{2}^{p,-q}=H^{p}\left(F, \pi_{q} \mathcal{E}_{\bar{F}}\right) \Rightarrow \pi_{q-p} \mathbb{H}^{\cdot}(F, \mathcal{E})
$$

Our main example is the case $\mathcal{E}^{n}=\mathcal{K}(-) \wedge M \mathbb{Z} / \ell^{n}$, where $\mathcal{K}$ is the $\mathrm{K}$ theory presheaf. Then Suslin's theorem yields

Corollary 1.5 For any field $F$ and prime $\ell$, there is a conditionally convergent fourth quadrant cohomology spectral sequence

$$
E_{2}^{p,-q}=H^{p}\left(F ; Z_{\ell}\left(\frac{q}{2}\right)\right) \Rightarrow \pi_{q-p} \mathbb{H}^{\prime}(F ; \mathcal{K})^{\wedge}
$$

$\left(E_{2}^{p,-q}\right.$ is zero for $q$ odd or $q<0$.)

Note that Suslin's theorem is needed to identify the $E_{2}$-term. Also, we have implicitly used the fact that smashing with a finite spectrum commutes with hypercohomology, in order to make the identification

$$
\operatorname{holim}_{n} \mathbb{H}^{\cdot}\left(F, \mathcal{K} \wedge M \mathbb{Z} / \ell^{n}\right)=\operatorname{holim}_{n} \mathbb{H}^{\cdot}(F, \mathcal{K}) \wedge M \mathbb{Z} / \ell^{n}
$$

This will be justified in a more general context in section 3. An explanation of why we get continuous cohomology can be found in section 4.11.

Note that the spectral sequence of the corollary - the descent spectral sequence for K-theory - will converge if, for example, $c d_{\ell} F<\infty$. When $F=\mathbb{R}$ and $\ell=2$, we have $c d_{2} \mathbb{R}=\infty$ but one can show the spectral sequence converges nevertheless.

Note also that when $c d_{\ell} F=d<\infty$, we conclude that $\mathbb{H}^{\cdot}(F, \mathcal{K})^{\wedge}$ is $(-\mathrm{d}$ 1)-connected, and

$$
\pi_{-d} \mathbb{H}^{\cdot}(F, \mathcal{K})^{\wedge}=H^{d}\left(F ; Z_{\ell}(0)\right.
$$

This group can easily be nonzero - for example, if $\mathrm{F}$ is any finite field, $\ell$ any prime, we have $\pi_{-1}=Z_{\ell}$. 
For any presheaf $\mathcal{E}$, there is a natural augmentation map $\mathcal{E}(F) \rightarrow \mathbb{H}^{\cdot}(F ; \mathcal{E})$ (analogous to $X^{G} \rightarrow X^{h G}$ ). In this context, the Lichtenbaum-Quillen conjecture asserts:

Conjecture 1.6 If $c d_{\ell} F<\infty$, the augmentation $K F^{\wedge} \longrightarrow \mathbb{H}^{\cdot}(F, \mathcal{K})^{\wedge}$ is a weak equivalence on some connected cover.

There are some explicit conjectures as to what the isomorphism range is. Clearly one must at least pass to the (-1)-connected cover of the hypercohomology spectrum - but this is not enough. Suppose for example that $c d_{\ell} F \leq 2$. Then the spectral sequence collapses and

$$
\pi_{0} \mathbb{H}^{\cdot}(F, \mathcal{K})^{\wedge} \cong Z_{\ell} \oplus H^{2}\left(F ; Z_{\ell}(1)\right)
$$

The term $H^{2}\left(F ; Z_{\ell}(1)\right)$ is closely related to the Brauer group and is typically nonzero. Hence in this case, there is at best an isomorphism above dimension zero.

\subsection{Statement of Thomason's Descent Theorem for Fields}

Definition 1.7 A field $F$ is $\ell$-good if char $F \neq \ell$ and $F$ has finite transcendence degree over a subfield $E$ satisfying $c d_{\ell} E_{\infty} \leq 1$, where $E_{\infty}$ is obtained from $E$ by adjoining all the $\ell$-power roots of unity. If $\ell=2$ we also require $\sqrt{-1} \in E$.

The condition $\sqrt{-1} \in F$ when $\ell=2$ is needed for two reasons. First of all, we will need to know that $c d_{\ell} F<\infty$. For that we could get by with the weaker assumption $c d_{\ell}\left(E_{\infty} / E\right) \leq 1$. However one also needs $\sqrt{-1} \in$ $F$ for the construction of Bott elements. Examples of fields $E$ as above include separably closed fields, finite fields, local fields, and number fields (with $\sqrt{-1} \in F$ if $\ell=2$ ). Note that an $\ell$-good field has finite $c d_{\ell}$.

Theorem 1.8 Let $F$ be an $\ell$-good field. Then the natural augmentation $K F \longrightarrow \mathbb{H}^{\cdot}(F, \mathcal{K})$ induces a weak equivalence

$$
\hat{L} K F \cong \hat{L} \mathbb{H}^{\cdot}(F, \mathcal{K})
$$


and there is a convergent spectral sequence

$$
E_{2}^{p,-q}=H^{p}\left(F ; Z_{\ell}\left(\frac{q}{2}\right)\right) \Rightarrow \pi_{q-p} \hat{L} K F
$$

One can show that the functor L commutes with hypercohomology (see Corollary 3.12), so $\hat{L} \mathbb{H}^{\cdot}(F, \mathcal{K})=\left(\mathbb{H}^{\cdot}(F, L \mathcal{K})\right)^{\wedge}$. Then the spectral sequence is obtained as before.

The theorem can be reformulated in terms of "Bott-periodic" K-theory. Let $k F=K F \wedge M \mathbb{Z} / \ell$. Then the theorem is equivalent to the assertion that $L k F \longrightarrow \mathbb{H}^{\cdot}(F ; L k)$ is a weak equivalence, and moreover an easy transfer argument reduces to the case when $\mathrm{F}$ contains $\ell$-th roots of unity. In that case we fix once and for all a Bott element $\beta \in \pi_{2} k F$. Then by a theorem of Snaith and Dwyer, $L k F \cong \beta^{-1} k F$. Theorem 1.8 is then equivalent to:

Theorem 1.9 Let $F$ be an $\ell$-good field containing $\ell$-th roots of unity. Then the natural augmentation $k F \longrightarrow \mathbb{H}^{\cdot}(F, k)$ induces a weak equivalence

$$
\beta^{-1} k F \cong \beta^{-1} \mathbb{H}^{\cdot}(F ; k)
$$

The proof of this theorem will be sketched in the final section.

\section{Schemes, Sites and Cohomology}

This section is directed strictly from one non-expert to another. Its purpose is to provide, in condensed form, the technical background on sites and cohomology necessary to penetrate [42] and [22].

Just keeping track of the scheme-theoretic hypotheses in [42] is already a non-trivial task. The most basic assumption will usually be that " $\mathrm{X}$ is a separated noetherian regular scheme, of finite Krull dimension", so we briefly review these notions in the first subsection. In the next two subsections we recall, for the reader's convenience, the definitions of étale morphisms and hensel local rings.

In the fourth subsection we introduce Grothendieck sites. A site is a beautiful and far-reaching generalization of a topological space. It is an extremely general object, which at the same time is far simpler than any of its special cases. The examples of most importance for this paper are the 
étale, Nisnevich and Zariski sites of a scheme. The Nisnevich site lies between the étale and Zariski sites, and serves to bridge the gap between fields and general schemes. Next we introduce presheaves and sheaves, and discuss the various direct and inverse image functors associated to a site morphism.

The topos associated to a site is its category of sheaves of sets. According to [3] (IV, introduction) or [23] (introduction), it is the topos that is of paramount importance - different sites can yield the same topos, and a choice of site is just a particular way to construct the topos. Be that as it may, it is not the point of view that will be taken here. Our ultimate goal is to study the homotopy theory of presheaves of spectra on a site, and it would seem to be highly desirable - even essential - to work on an actual site rather than the disembodied topos. Thus topos theory per se will make only a minor appearance.

One place where the topos does enter is in the next section, on points and stalks. There is a beautiful theory ([3], IV.6) extending the concept of stalk at a point, familiar from classical sheaf theory, to general sites. The idea is that it is the inverse image or stalk functor on sheaves - associated to inclusion of a point in the usual sense - which carries all the essential information. Formulated in this way, the classical theory of points and stalks carries over to general sites to a remarkable degree. The most noteworthy difference is that a given site may or may not have "enough points", meaning that stalks detect isomorphisms in the usual way. The étale, Nisnevich and Zariski sites have enough points.

The next two subsections discuss sheaf cohomology, including some results on étale and Nisnevich cohomological dimension that are crucial for the applications to spectra. Then we introduce $\check{C}$ ech cohomology and compare it with sheaf cohomology.

In the final section on noetherian sites we discuss two crucial results on commuting sheaf cohomology with filtered colimits. The first of these involves colimits in the sheaf variable, and is straightforward. The second result - the so-called "Continuity Theorem" has to do with co-filtered inverse limits in the scheme variable, with the étale topology (or more generally inverse limits of sites, but discretion seems the better part of valor here). This theorem is somewhat more difficult to state, and much more difficult to prove. In the affine case it says roughly that étale cohomology commutes with filtered colimits of rings.

For a quick introduction to sites and étale cohomology, see [41] or [1]. 
The definitive treatment is the original source [3]. For the Nisnevich site see [34], [25], Appendix E in [44] and Chapter 7 of [22].

\section{$2.1 \quad$ Schemes}

We recall that a scheme $\mathrm{X}$ is separated if the diagonal $\Delta_{X}$ is closed in $X \times X$. For example any affine scheme or algebraic variety is separated, whereas e.g. affine n-space "with a point doubled" is not. More generally a morphism $X \longrightarrow Y$ is separated if $\Delta_{X}$ is closed in $X \times_{Y} X$. Separation hypotheses enter in various ways. For example, in general the intersection of two affine open subsets need not be affine (consider the example above with $n \geq 2$ ), but this is easily checked to be true for separated schemes. Another point is that valuations behave well on separated schemes ([18],II.6, remark at the bottom of p.130). General considerations like these lie behind the separated hypothesis in the resolution theorem below.

A scheme $\mathrm{X}$ is noetherian if it has a finite open affine cover

$$
X=\cup \operatorname{Spec} A_{i}
$$

with $A_{i}$ a noetherian ring. This implies that the underlying space of $\mathrm{X}$ is noetherian and in particular quasi-compact ([18], II, Ex. 2.13). The noetherian hypothesis is convenient for mostly obvious reasons. In some cases it could be replaced by "locally noetherian" - or even omitted entirely, provided that "finite-type" assumptions are replaced by their "finitely-presented" analogues. We will usually assume that schemes are at least locally noetherian. For a general discussion of finiteness hypotheses, beyond the noetherian condition, see [16],6.

The Krull dimension of $\mathrm{X}$ is the maximal length $n$ of a descending chain

$$
X_{0} \supset X_{1} \supset \ldots \supset X_{n}
$$

of irreducible closed subsets. It's worth pointing out here that although local noetherian rings always have finite Krull dimension, this isn't true in general (see [12], exercise 9.6). Hence our typical hypothesis "let X be a separated noetherian scheme of finite Krull dimension" is not redundant.

$\mathrm{X}$ is regular if all of its local rings $\mathcal{O}_{x}$ are regular. Here a local ring $\mathrm{R}$ with maximal ideal $m$ and residue field $k=R / m$ is regular if it is noetherian and $\operatorname{dim}_{k}\left(m / m^{2}\right)=$ Krull dim X. For example, Dedekind domains are regular, and 
an algebraic variety over a field is regular if and only if it is smooth. The key property of regular schemes that we need is (see for example [18],III,exercise $6.9)$.

Theorem 2.1 Let $X$ be a separated noetherian regular scheme. Then every coherent sheaf on $X$ admits a finite resolution by locally free sheaves.

We remind the reader that coherent (resp. locally free) sheaves correspond to finitely-generated (resp. finitely-generated projective) modules in the affine case. Theorem 2.1 is the ultimate source of the separated and regular hypothesis that occurs in several of the main theorems below.

\section{2 Étale morphisms}

A morphism of locally noetherian schemes $X \stackrel{f}{\longrightarrow} Y$ is étale if it is

- flat: for all $x \in X$ the induced homomorphism $\mathcal{O}_{f(x)} \longrightarrow \mathcal{O}_{x}$ of local rings is flat

- unramified: the fibre of $f$ over Spec $k=y \in Y$ is a finite disjoint union of finite separable extensions $S p e c k_{i}$ of the residue field of $y$. In other words, there is a pullback diagram

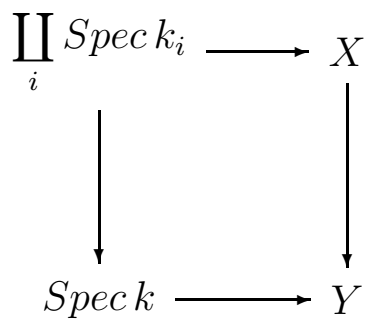

- locally of finite type: There is an affine open cover $U_{i}$ of $\mathrm{Y}$ and for each $i$ an affine open cover $V_{i j}$ of $f^{-1} U_{i}$ such that the ring homomorphism corresponding to each $V_{i j} \longrightarrow U_{i}$ has finite type. (For general schemes one requires that $f$ is locally of finite presentation; see e.g. [41].)

The "unramified" condition has an alternative formulation. Let $R \rightarrow S$ be a map of rings, $\Omega_{S / R}$ the "module of relative Kahler differentials" (i.e. the R-module on symbols $d s, s \in S$ modulo the Leibniz rule - see [18],II.8). Then 
Spec $S \longrightarrow$ Spec $R$ is unramified if and only if $\Omega_{S / R}=0$, with a similar result holding for arbitrary schemes (cf. [29],I,3.5).

We will invariably assume that our étale maps actually have finite type; this just means that in the third condition above we have for each fixed $i$ a finite such cover $V_{i j}$ (and since our schemes will usually be noetherian, the cover $U_{i}$ will also be finite).

The basic examples of an étale morphism that the reader should keep in mind are the following:

- A smooth unramified covering map between complex algebraic varieties

- The morphism Spec $L \longrightarrow$ Spec F corresponding to finite separable field extension $\mathrm{L} / \mathrm{F}$

- The morphism Spec $S \longrightarrow S p e c R$ corresponding to an unramified finite extension of Dedekind domains

In the first and third examples, one should picture more generally a map which is not onto but rather has image some open subset of the target. For example in the case of an arbitrary finite extension of Dedekind domains, the associated map of schemes is étale over the complement of the finite set of ramified primes.

We note that étale maps are stable under base change and composition, any open immersion is étale, and any étale map is an open map.

\subsection{Hensel local rings}

Let $\mathrm{R}$ be a local ring with maximal ideal $m$ and residue field $k$. Then $\mathrm{R}$ is a hensel local ring, or is henselian, if every finite R-algebra $\mathrm{S}$ splits as a finite product of local R-algebras. This is equivalent to R satisfying "Hensel's lemma" on lifting factorizations of polynomials from $k$ to $\mathrm{R}$. Any local ring $\mathrm{R}$ admits a henselization $R \longrightarrow R^{h}$ which is initial among local homomorphisms to henselian rings. One can think of henselization as a kind of algebraic approximation to the analytic process of completion. Every complete local ring is henselian, and in general $R^{h}$ sits between $\mathrm{R}$ and the completion of $\mathrm{R}$.

If $\mathrm{S}$ is a finite $\mathrm{R}$-algebra and is étale over $\mathrm{R}$, then $\mathrm{S}$ splits as a product of local R-algebras which are themselves henselian. 
Theorem 2.2 Let $R$ be a hensel local ring. Then the functor $S \mapsto S \otimes_{R} k$ is an equivalence between the category of finite étale $R$-algebras and the category of finite étale $k$-algebras.

A strict hensel (or strictly henselian, or "strictly local") ring) is a hensel local ring $\mathrm{R}$ with separably closed residue field. Note that in this case, the preceeding theorem says that every finite étale $\mathrm{R}$-algebra splits as a product of copies of $\mathrm{R}$.

There is also a strict henselization functor $R \longrightarrow R^{s h}$. For further discussion see section 2.6 below.

\subsection{Sites}

A site $\Phi=(\mathcal{C}, \mathcal{T})$ consists of (1) a category $\mathcal{C}$ with pullbacks; and (2) a Grothendieck topology $\mathcal{T}$ on $\mathcal{C}$ - that is, for each object $\mathrm{U}$ of $\mathcal{C}$ a collection of families of morphisms $\left\{U_{i} \longrightarrow U\right\}$ called covering families, such that every cover of a cover is a cover, every pullback of a cover is a cover, and every family consisting of a single isomorphism is a cover. All sites considered in this paper will also have terminal objects and hence have all finite limits.

The reader can easily guess the precise statements needed in (2). See e.g. [1], [41], or the original source [3]. We remark that the definition of site given here is less general than the usual one.

There are several examples that will be important for us. Let $\mathrm{X}$ be a locally noetherian scheme.

- The Zariski site $X_{Z a r}$. The underlying category $\mathcal{C}$ is the category of open subsets of $\mathrm{X}$ (for the Zariski topology), with morphisms the inclusions. A family $\left\{U_{i} \longrightarrow U\right\}$ is a covering family if the $U_{i}$ cover $\mathrm{U}$ in the usual sense. This site makes sense for any topological space, and is the motivating example.

- The étale site $X_{e ́ t} . \mathcal{C}$ is the category of schemes étale and of finite type over X. A family $\left\{U_{i} \longrightarrow U\right\}$ is a covering family if the images of the $U_{i}$ cover $\mathrm{U}$ in the usual sense.

- The restricted étale site $X_{\text {rét }}$ of a separated noetherian scheme. This is defined as in the previous example, except that we require the scheme over $\mathrm{X}, U \longrightarrow X$ to be separated over $\mathrm{X}$, and the covering families are 
to have only finitely many elements $U_{i} \longrightarrow U$. (It follows automatically that $U_{i}$ is separated over $U$ as well.) One advantage of using the restricted site is that a covering family $\left\{U_{i} \longrightarrow U\right\}$ can be regarded as a single surjective étale map $U^{\prime} \longrightarrow U$, where $U^{\prime}=\amalg U_{i}$. For the restricted étale site of a general scheme, see [41],II,1.5.

- The Nisnevich site $X_{N i s}$ [34]. The underlying category is the same as for the étale site. To describe the covering families, we borrow a term from number theory. Given an étale $h: U^{\prime} \longrightarrow U$, we say that $x \in U$ is completely decomposed in $U^{\prime}$ if there is a point $y \in h^{-1} x$ such that the induced map on residue fields $k(x) \longrightarrow k(y)$ is an isomorphism. A covering family for the Nisnevich topology is a covering family $\left\{U_{i} \longrightarrow U\right\}$ for the étale topology such that for all $x \in U, x$ is completely decomposed in at least one $U_{i}$. This site will be discussed further in section 2.6.

- The site $G_{f s e t s}$ attached to a profinite group $G$. A G-set is discrete if all its isotropy groups in $\mathrm{G}$ are open. We take for $\mathcal{C}$ the discrete finite G-sets. Covering families are just the surjective families.

- "Big" sites. The étale site discussed above is analogous to a single topological space. The étale morphisms $U \rightarrow X$ play the role of the open subsets. But we could also consider some much larger category of schemes - even all schemes - and still get a Grothendieck site, using the same covering families. For example we could take all schemes X locally of finite type over a fixed base scheme $\mathrm{S}$, and equip each $\mathrm{X}$ with the étale topology. This kind of a site (or its Zariski or Nisnevich analogue) is roughly analogous to the category of all topological spaces, and will be informally referred to as a "big", or "sufficiently large" site.

Two further examples can be found in the next section.

A morphism of sites $f: \Phi \longrightarrow \Phi^{\prime}$ consists of a functor $f^{-1}: \mathcal{C}^{\prime} \longrightarrow \mathcal{C}$ that preserves pullbacks and preserves covering families in the obvious sense. ${ }^{3}$ If the sites have terminal objects, and $f^{-1}$ preserves them, then $f^{-1}$ preserves all finite limits. This will be the case in the examples considered in this paper, with one exception discussed in Section 2.5 below. An equivalence

\footnotetext{
${ }^{3}$ The notation $f^{-1}$ for the functor is meant to be suggestive - perhaps dangerously so, but we're going to take the risk.
} 
of sites is a morphism such that $f^{-1}$ is an equivalence of categories and any inverse functor is also a site morphism. Exercise: show that if $\mathrm{F}$ is a field, the site $\left(G_{F}\right)_{f s e t s}$ is equivalent to the étale site of $\mathrm{F}$.

Our convention that the site morphism goes in the opposite direction is in agreement with some sources but not with others (e.g. [1] or [41], in which our morphism $f$ would be written as a "morphism of topologies" $\mathcal{T}^{\prime} \longrightarrow \mathcal{T}$ ). The reason for the convention is just that a morphism of schemes $X \longrightarrow Y$ then induces site morphisms in the same direction $X_{Z a r} \longrightarrow Y_{Z a r}, X_{\text {ét }} \longrightarrow Y_{\text {ét }}$, etc., and a continuous homomorphism $G \longrightarrow H$ of profinite groups induces a morphism $G_{f s e t s} \longrightarrow H_{f s e t s}$. On the other hand it must be admitted that there are some other cases where our convention looks slightly odd. For example, it is clear that $X_{Z a r}$ and $X_{N i s}$ are "sub-sites" of $X_{e ́ t}$, etc., but in our notation the evident site morphisms go the other way:

$$
X_{\text {ét }} \longrightarrow X_{N i s} \longrightarrow X_{Z a r}
$$

However even in this case, our convention helps the wandering topologist to keep track of things such as the Leray spectral sequence described below. Site morphisms associated to inclusion of a sub-site as above will be called restriction morphisms.

\subsection{Presheaves and sheaves}

A presheaf of sets on a site $\Phi=(\mathcal{C}, \mathcal{T})$ is just a contravariant functor from $\mathcal{C}$ to sets. An abelian presheaf is a presheaf of abelian groups. Similarly one can define presheaves with values in any category (simplicial sets, spectra, etc.), but for now we will focus on presheaves of sets or abelian groups. A sheaf is a presheaf $\mathcal{E}$ that satisfies the usual type of exactness condition with respect to covering families.

There is an exact sheafification functor $a$, left adjoint to the inclusion functor $i$ of presheaves in sheaves. ${ }^{4}$ A presheaf of sets $\mathcal{E}$ is representable if $\mathcal{E}(U)=\operatorname{Hom}(U, W)$ for some object W. If $\mathcal{C}$ is an arbitary category with pullbacks, the finest topology such that every representable presheaf is a sheaf is called the canonical topology - see e.g. [41]. On most of the sites encountered in practice, every representable presheaf is a sheaf - in

\footnotetext{
${ }^{4}$ Later, when we consider presheaves of homotopy groups $\pi_{q} \mathcal{E}$, the associated sheaf will be denoted $\tilde{\pi}_{q} \mathcal{E}$.
} 
other words, the topology in question is no finer than the canonical topology. In the general case the phrase "sheaf represented by W" secretly means the sheafification of $\operatorname{Hom}(-, W)$. The category $\mathcal{C}$ also admits a minimal topology, called the "chaotic" topology, in which the only covering families are the isomorphisms. In this topology, every presheaf is a sheaf.

Given a site morphism $f: \Phi \longrightarrow \Phi^{\prime}$, one can define various direct and inverse image functors on sheaves and presheaves. Since these functors are extremely important, and keeping them all straight gets a bit confusing, we will make a few remarks that may be of help.

Consider a functor $g: I \longrightarrow J$ between small categories. Let $\mathcal{D}$ be another category, which has all limits and colimits, and consider the restriction functor $\mathcal{D}^{g}: \mathcal{D}^{J} \longrightarrow \mathcal{D}^{I}$ on functor categories (or "diagrams"). This functor has both a left adjoint $G_{L}$ and a right adjoint $G_{R}$, also know as Kan extensions $([26], \mathrm{X})$. Bearing in mind that presheaves are contravariant functors, we can apply this to our site morphism $f$, with $g=f^{-1}$. We write $f_{\#}$ (direct image) for the restriction functor, $f^{\#}$ (inverse image) for the left adjoint and $f^{!}$for the right adjoint. Thus if $\mathcal{E}$ is a presheaf on $\Phi, f_{\#} \mathcal{E}(U)=\mathcal{E}\left(f^{-1} U\right)$. And if $\mathcal{F}$ is a presheaf on $\Phi^{\prime}, f^{\#} \mathcal{F}(W)=\operatorname{colim}_{V} \mathcal{F}(V)$, where the colimit is taken over the opposite of the over-category $\left(f^{-1} \downarrow U\right)$. If the sites have terminal objects and $f^{-1}$ preserves them, $\left(f^{-1} \downarrow U\right)^{o p}$ is filtered. Otherwise it is only pseudofiltered.

One would like to promote these functors to the sheaf level. There is no problem with the direct image functor because it preserves sheaves and so immediately yields a direct image functor on sheaves, denoted $f_{*}$. On the other hand the symmetry between left and right adjoints breaks down. The reason is clear - the sheafification functor $a$ is a left adjoint. Hence, even though $f^{\#}$ may fail to preserve sheaves, we get a left adjoint to $f_{*}$ by setting $f^{*}=a f^{\#} i$. The analogous attempt to extend $f^{!}$fails simply because $s$ appears on the wrong side. One nevertheless gets a right adjoint $f^{!}$on the sheaf level in certain situations - e.g. inclusion of a closed subscheme in the Zariski or étale topologies.

To confuse matters still further, there are situations where we have site morphisms going in both directions. Specifically, consider a fixed object U of a site that has a terminal object $\mathrm{X}$. Define the local site $\Phi / U$ to have underlying category the category of objects over $U$ and covering families the same as those in $\Phi$. The simple example to keep in mind is an open subscheme in the Zariski topology. There is an obvious site morphism $j: \Phi / U \longrightarrow \Phi$ with 
$j^{-1} V=U \times_{X} V$. But there is another obvious site morphism $k: \Phi \longrightarrow \Phi / U$ with $k^{-1}(V \rightarrow U)=V$. (Note that $k^{-1}$ does not preserve terminal objects or products.) Inspection shows that $j^{\#}=k_{\#}$. Hence $j^{\#}$ preserves sheaves and has a left adjoint $j_{!}\left(=k^{\#}\right)$, called "extension by zero" because in the Zariski example mentioned above we have $j_{!} \mathcal{E}(V)=\mathcal{E}(V)$ if $V \subset U$ and $j_{!} \mathcal{E}(V)=0$ otherwise. In general we have

$$
j_{!} \mathcal{E}(V)=\coprod_{H o m(V, U)} \mathcal{E}(V)
$$

The category of presheaves on the site $\Phi$ is sometimes denoted $\Phi^{\wedge}$. The category of sheaves (the "topos") is sometimes denoted $\Phi^{\sim}$.

\subsection{Points and stalks}

In this section presheaves and sheaves will be set-valued unless otherwise mentioned.

In classical sheaf theory, on the Zariski site of a scheme $\mathrm{X}$, the notion of "stalk" at a point $x \in X$ is extremely useful. For example, stalks detect isomorphisms in the sense that a map of sheaves is an isomorphism if and only if it induces an isomorphism on all stalks. It isn't obvious how to define stalks on an arbitrary site, because it isn't even clear what a "point" should be. The key is to observe that the category of sheaves on the Zariski site of $x=S p e c k$ is the category of sets, and that the corresponding stalk functor $X_{Z} \tilde{a r} \longrightarrow S e t s$ is a left adjoint that commutes with finite limits. Thus on a site $\Phi$ we define a point $p$ as a pair of adjoint functors

$$
p^{*}: \Phi^{\sim} \longrightarrow \text { Sets, } \quad p_{*}: \text { Sets } \longrightarrow \Phi^{\sim}
$$

where the left adjoint $p^{*}$ is required to commute with finite limits. The stalk of a sheaf $F$ is then defined by $F_{p}=p^{*} F$. In fact the point $p$ is determined by a functor $p^{*}$ that commutes with finite limits and all colimits, because the right adjoint exists automatically. Indeed one has the explicit formula for a set $S$

$$
p_{*} S(U)=\operatorname{Hom}_{\text {sets }}\left(p^{*} \tilde{U}, S\right)
$$

which is easily verified using the colimit formula below for $p^{*}(\tilde{U}$ is the sheaf represented by the object $\mathrm{U})$. 
A neighborhood of a point $p$ is a pair $(U, a)$ with $U$ an object of the site and $a \in p^{*} \tilde{U}$. Equivalently, this can be thought of as a lift of the point to the local site $\Phi / U$. The neighborhoods form a category $\mathcal{N}_{p}$ in an obvious way, and this category is co-filtered. For any sheaf $\mathrm{F}$ we have

$$
F_{p}=\operatorname{colim}_{\mathcal{N}_{p}} F(U)
$$

or more generally for a presheaf $\mathrm{F}$

$$
a F_{p}=\operatorname{colim}_{\mathcal{N}_{p}} F(U)
$$

A set of points of a site is said to be sufficient if it detects isomorphisms of sheaves in the sense described above. A site has enough points if it has a sufficient set of points. Not all sites have enough points. ${ }^{5}$ However there is the following elegant and useful criterion ([3],IV,6.5). We say that a set of points of $\Phi$ detects covering families provided that whenever $\left\{U_{i} \longrightarrow U\right\}$ is a family of morphisms and $\left\{p^{*} U_{i} \longrightarrow p^{*} U\right\}$ is a surjective family of sets for all points $p$ in the set, then $\left\{U_{i} \longrightarrow U\right\}$ is a covering family. Then the criterion states that a set of points of $\Phi$ is sufficient if and only if it detects covering families.

In the case of the Zariski site, one can show that the new definitions agree with the old - although it isn't obvious that every site-theoretic point comes from a classical point (see [23],7.24, for a quick proof). Note that in this case the stalk of the structure sheaf is the Zariski local ring $\mathcal{O}_{x}$.

Now consider the étale site of a scheme X. Given an ordinary point $x \longrightarrow X, x=$ Speck, choose a separable closure $\bar{x}=$ Spec $\bar{k}$. Then the category of sheaves on $\bar{x}_{e t}$ is the category of sets, and the site morphism $p: \bar{x}_{e t} \longrightarrow X$ induces a functor $p^{*}$ on sheaves that commutes with finite limits and all colimits. Thus each such "geometric point" $\bar{x} \longrightarrow X$ defines a sitetheoretic point of $X_{e t}$. One can easily check that a neighborhood of $\bar{x} \longrightarrow X$ is the same thing as an étale $U \longrightarrow X$ together with a lift

\footnotetext{
${ }^{5}$ See [3],IV,7.4 for an interesting example -involving Lebesgue measure on the unit interval - of a site with no points.
} 


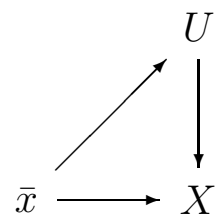

It follows that the stalk at $\bar{x}$ of the structure sheaf $U \longrightarrow \mathcal{O}_{U}$ is the strict henselization $\mathcal{O}_{\bar{x}}^{s h}$ of $\mathcal{O}_{x}$. Thus the rings $\mathcal{O}_{\bar{x}}^{s h}$ are the local rings for the étale topology. Finally, it is easy to see that geometric points detect covering families, so the étale site has enough points.

Contemplating the vast gap between the Zariski local ring $\mathcal{O}_{x}$ and its strict henselization $\mathcal{O}_{x}^{s h}$, one longs for an intermediate world in which the local rings are the ordinary henselizations $\mathcal{O}_{x}^{h}$. The Nisnevich site is precisely such a world. ${ }^{6}$

Consider first the Nisnevich site of $x=S p e c k, k$ a field. Call a presheaf $\mathrm{F}$ additive if $F(U \amalg V)=F(U) \times F(V)$. Then it is easily seen that the sheaves on $x_{N i s}$ are precisely the additive presheaves (in effect, any covering family takes the form $U \amalg W \longrightarrow U)$. Hence sheaves are the same thing as presheaves on the full subcategory of connected étale $x$-schemes. In particular, for any such scheme $x^{\prime}$ (i.e. $x^{\prime}=$ Spec $k^{\prime}$, with $k^{\prime}$ a finite separable extension of $k$ ), the sections functor $F \longrightarrow F\left(x^{\prime}\right)$ is exact.

For a general scheme $\mathrm{X}$, consider an ordinary point $i: x \longrightarrow X$, and $x^{\prime} \longrightarrow x$ as above. Define $p^{*}: X_{N i s}^{\sim} \longrightarrow$ Sets by $p^{*} F=j_{N i s}^{*} F\left(x^{\prime}\right)$, where $j: x^{\prime} \longrightarrow X$. Then, thanks to the exactness of the sections functor, $p^{*}$ defines a point of $X_{N i s}$. Informally, then, a point of the Nisnevich site is denoted $x^{\prime} \longrightarrow x$ or $x^{\prime} \longrightarrow X$. A neighborhood of such a point is the same thing as a commutative diagram

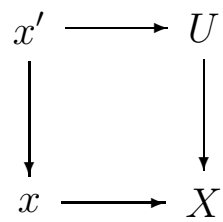

\footnotetext{
${ }^{6}$ As will be seen, however, the Nisnevich topology is much closer to the Zariski topology than it is to the étale topology.
} 
Note that $x^{\prime}$ need not be a residue field of $\mathrm{U}$ - in general, it is only a finite extension of such a field. However the neighborhoods for which $x^{\prime}$ is a residue field of $U$ form a cofinal subcategory of $\mathcal{N}_{p}$.

It follows that the Nisnevich stalk at $x \in X$ of the structure sheaf is $\mathcal{O}_{x}^{h}$, the henselization of $\mathcal{O}_{x}$. More generally the stalk at $x^{\prime} \longrightarrow x$ is $\mathcal{O}_{x^{\prime}}^{h}$, the hensel extension of $\mathcal{O}_{x}^{h}$ corresponding to $x^{\prime} \longrightarrow x$ under the equivalence of Theorem 2.2. We will call this ring the "henselization of $\mathcal{O}_{x}$ at $x^{\prime} \longrightarrow x$ ". Thus the local rings for the Nisnevich topology are the henselizations $\mathcal{O}_{x^{\prime}}^{h}$.

Finally, it is easy to check that the points described above detect covering families, and hence the Nisnevich site has enough points.

\subsection{Sheaf Cohomology}

Let $\mathrm{A}$ be an abelian sheaf, $\mathrm{U}$ an object of $\mathcal{C}$. The category of sheaves is abelian with enough injectives, and the sheaf cohomology groups $H^{p}(U ; A)$ are the p-th derived functors of the left exact "sections on U" functor $U \longrightarrow A(U)$. For the sites $X_{Z a r}, X_{e ́ t}$ and so on we will write this as $H_{Z a r}^{p}(U ; A), H_{e ́ t}^{p}(U ; A)$ etc.

When the site has enough points, there is a functorial Godement resolution defined as follows. For each point $p$, the adjoint functors $p^{*}, p_{*}$ defined a monad $([26], \mathrm{VI})$, and any monad defines a cosimplicial object in a standard way. Here we will take the product over a sufficient set of points, obtaining a monad on sheaves given by

$$
T(A)=\prod_{p} p_{*} p^{*} A
$$

The resulting cosimplicial sheaf $T^{\cdot}(A)$ provides a resolution of $A$ by sheaves which, although not necessarily injective, are "flasque" and in particular acyclic for cohomology. Hence, taking sections over $U$ of the Godement resolution yields a functorial complex for computing cohomology.

One of the main tools for computing sheaf cohomology is the fantastically general "Leray" or "change-of-site" spectral sequence:

Theorem 2.3 Let $f: \Phi \longrightarrow \Phi^{\prime}$ be a site morphism, $A$ a sheaf on $\Phi$. Then for any object $U$ in $\mathcal{C}$ there is a first quadrant cohomology spectral sequence

$$
E_{2}^{p, q}=H^{p}\left(U ; R^{q} f_{*} A\right) \Rightarrow H^{p+q}\left(f^{-1} U ; A\right)
$$


Here the $R^{q} f_{*} A$ are the "higher direct image sheaves" which are the derived functors of $f_{*}$. They can be described as the sheafifications of the presheaves $U \longrightarrow H^{q}\left(f^{-1} U ; A\right)$. In the case of a morphism of schemes $X \longrightarrow Y$, we would typically take $\mathrm{Y}=\mathrm{U}, X=f^{-1} U$ and get the classical Leray spectral sequence of $X_{Z a r} \longrightarrow Y_{Z a r}$, its counterpart for the étale site, etc. For the site morphism associated to a surjective homomorphism of profinite groups, we get the Hochshild-Serre spectral sequence.

Now consider the case of a "restriction" morphism such as $X_{e ́ t} \longrightarrow X_{Z a r}$. In this case the "direct image" functor is just the obvious restriction of sheaves to the smaller category. Nevertheless one gets a very interesting spectral sequence. For example in the étale-to-Zariski case one easily derives the generalized "Hilbert's Theorem 90" asserting that

$$
H_{e ́ t}^{1}\left(X, G_{m}\right)=H_{Z a r}^{1}\left(X, G_{m}\right)=\operatorname{Pic} X
$$

One can also use the spectral sequence to show that a given sub-site has the same cohomology as the ambient site. We'll call the following result the "weak comparison lemma" ([41], 3.9.3).

Proposition 2.4 Let $f: \Phi \longrightarrow \Phi^{\prime}$ be a restriction morphism of sites. Suppose that for every object $U$ in $\mathcal{C}^{\prime}$ and every covering family $\left\{V_{i} \longrightarrow U\right\}$ in the ambient site $\Phi$, there are covering families $\left\{U_{i j} \longrightarrow V_{i}\right\}$ with $U_{i j}$ in $\mathcal{C}^{\prime}$ and $\left\{U_{i j} \longrightarrow U\right\}$ in $\mathcal{T}^{\prime}$. Then for any sheaf $A$ on $\Phi$ and object $U$ in $\Phi^{\prime}$,

$$
H_{\Phi^{\prime}}^{*}\left(U, f_{*} A\right) \cong H_{\Phi}^{*}(U, A)
$$

This applies for example to the restriction from the étale site of a separated noetherian scheme $\mathrm{X}$ to its restricted site as above. In fact in this case a stronger condition holds and one can apply the usual "comparison lemma" ([41], 3.9.1) to conclude the actual sheaf categories are equivalent. Another illustration is provided by the following important theorem:

Theorem 2.5 Let $R$ be a Hensel local ring with maximal ideal $m$ and residue field $R / m=k$. Then for any sheaf $A$ on $(\text { Spec } R)_{\text {ét }}$, the morphism $j$ : Speck $\longrightarrow$ Spec $R$ induces an isomorphism

$$
j^{*}: H_{e t}^{*}(\operatorname{Spec} R ; A) \cong H_{e t}^{*}\left(\operatorname{Spec} k, j^{*} A\right)
$$


The proof of this fact doesn't use the Leray spectral sequence of $j$. Instead one constructs a site morphism going the other way, as follows. Let us abbreviate $X=(\operatorname{Spec} R)$ and $x=($ Spec $k)$. Let $\Phi$ denote the sub-site of $X_{e ́ t}$ in which both the objects and covering families consist of a single map $Y \rightarrow X$ corresponding to a finite étale $\mathrm{R}$-algebra $\mathrm{S}$. Then we have site morphisms $X_{e ́ t} \longrightarrow \Phi \longrightarrow x_{e ́ t}$ with the first a cohomology isomorphism by the weak comparison lemma and the second an equivalence of sites. Letting $f$ denote the composite morphism, one has $f_{*}=j^{*}$ and the theorem follows easily.

Finally, the notion of continuous Galois cohomology discussed in section 1 extends immediately to an arbitrary Grothendieck site. The category of inverse systems of sheaves has enough injectives, and following [19] we define continuous cohomology as the derived functors of the composite functor lim 0 (global sections).

\subsection{Cohomological dimension}

We will frequently need to know that a scheme $\mathrm{X}$ has finite cohomological dimension for sheaves (or $\ell$-torsion sheaves) on the étale, Zariski or Nisnevich sites. In general, for an object $\mathrm{X}$ of a site $\Phi$, the cohomological dimension of $\mathrm{X}$ is the maximal $n$ (possibly infinite) such that there exists a sheaf $\mathrm{A}$ with $H_{\Phi}^{n}(X ; A) \neq 0$. The $\bmod \ell$ cohomological dimension is defined similarly, with the requirement that $\mathrm{A}$ is an $\ell$-torsion sheaf. In the case of a scheme $\mathrm{X}$, and one of the sites mentioned above, we will write this as $c d^{Z a r}(X), c d_{\ell}^{e ́ t} X$, etc.

The following theorem of Grothendieck applies to any noetherian topological space X (see [18], III, 2.7).

Theorem 2.6 Let $X$ be a noetherian scheme of Krull dimension $n$. Then $c d^{Z a r}(X) \leq n$.

The next result, due to Kato and Saito (see [34]), is another indication that the Nisnevich topology is not so far from the Zariski topology.

Theorem 2.7 Let $X$ be a noetherian scheme of Krull dimension $n$. Then $c d^{N i s}(X) \leq n$. 
In the étale case we need to pay attention to the cohomological dimension of the residue fields of $\mathrm{X}$. We say that $\mathrm{X}$ is uniformly $\ell$-bounded, with bound $d$, if for all residue fields $k(x)$ we have $c d_{\ell} k(x) \leq d$. Here $c d_{\ell}$ refers to Galois cohomological dimension, or equivalently $c d_{\ell}^{e ́ t} \operatorname{Spec} k(x)$.

Theorem 2.8 Let $X$ be a noetherian scheme of Krull dimension $n$, and suppose $X$ is uniformly $\ell$-bounded with bound $d$. Then $c d_{\ell}^{e ́ t} X \leq n+d$. In particular, $c d_{\ell}^{e ́ t} X$ is finite.

There is an elegant proof of this fact based on the Nisnevich site and the Kato-Seito theorem. We sketch it here as an illustration of how the Nisnevich site functions as an intermediary between fields and schemes.

Let $\mathrm{A}$ be an $\ell$-torsion sheaf on $X_{e ́ t}$, and consider the Leray spectral sequence of the site morphism $f: X_{e ́ t} \longrightarrow X_{N i s}$. We have

$$
E_{2}^{p, q}=H_{N i s}^{p}\left(X ; R^{q} f_{*} A\right)
$$

and hence $E_{2}^{p, q}=0$ for $p>n$ by the Kato-Saito theorem. On the other hand we claim that the sheaves $R^{q} f_{*} A$ on $X_{N i s}$ are identically zero for $q>d$, whence the result. It is equivalent to show that the stalks are zero. In the notation of section 2.6, the stalk at a point $x$ is the sheaf on $x_{N i s}$ that assigns to each $x^{\prime} \rightarrow x$ the group $H_{e t}^{q}\left(\mathcal{O}_{x^{\prime}}^{h} ; g^{*} A\right)$, where $\mathcal{O}_{x^{\prime}}^{h}$ is the hensel local ring attached to $x^{\prime}$ and $g$ is the natural map to $\mathrm{X}$ (here we have made use of the "continuity theorem" (Theorem 2.11 below)). But this is isomorphic to $H_{e ́ t}^{q}\left(x^{\prime} ; A\right)$, by 2.5. Since $k^{\prime} / k$ is a finite separable extension, $c d_{\ell} k^{\prime} \leq c d_{\ell} k$, and hence $H_{e ́ t}^{q}\left(x^{\prime} ; A\right)=0$ for $q>d$.

For example, suppose $\mathrm{X}$ is a variety of dimension $n$ over a field $k$ with $c d_{\ell} k<\infty$. Then the residue fields of $\mathrm{X}$ have transcendence degree at most $n$ over $k$. Hence by 1.3 , we get $c d_{\ell}^{e ́ t} X \leq 2 n+c d_{\ell} k$. Or if $\mathrm{X}$ is the ring of integers in a number field $\mathrm{F}$, with either $\ell$ odd or $\mathrm{F}$ pure imaginary, then $c d_{\ell}^{e ́ t} X \leq 3$.

Finally, we remark that the "uniformly $\ell$-bounded" hypothesis in the above theorem is inherited by any object $\mathrm{U}$ of $X_{\text {ét }}$ (with the same bound), because the residue fields of $U$ are finite separable extensions of the residue fields of $\mathrm{X}$. Hence if in addition $\mathrm{U}$ has finite type over $\mathrm{X}$, all the hypotheses of the above theorem are inherited by $\mathrm{X}$, and $c d_{\ell}^{e ́ t} U \leq n+d$. 


\section{9 Čech cohomology}

The usual definition of $\check{C}$ ech cohomology for spaces goes through essentially verbatim for an arbitrary Grothendieck site. Let $\mathrm{X}$ be an object of the site $\Phi, \mathrm{F}$ an abelian presheaf. Given a covering family $\mathcal{U}=\left\{U_{i} \longrightarrow U\right\}$, one forms the associated augmented $\check{C}$ ech complex

$$
0 \longrightarrow F(U) \longrightarrow \prod_{i} F\left(U_{i}\right) \longrightarrow \prod_{i, j} F\left(U_{i} \times_{U} U_{j}\right) \longrightarrow \ldots
$$

The cohomology of this complex (after removing $\mathrm{F}(\mathrm{U})$ ) is denoted $\check{H}^{*}(\mathcal{U} / X ; F)$ and called $\check{C}$ ech cohomology with respect to the cover $\mathcal{U}$. $\check{C}$ ech cohomology proper is defined by passing to a filtered colimit over covers (with the caveat that the covers themselves do not form a co-filtered category under refinement - see for example [41]), and denoted $\check{H}(X ; F)$.

It is not hard to see that the groups $\check{H}^{*}(\mathcal{U} / X ; F)$ are the derived functors of $\check{H}^{0}(\mathcal{U} / X ; F)$. Since $\check{H}^{0}(\mathcal{U} / X ; F)=F(X)$ if $\mathrm{F}$ is a sheaf, the global sections functor for sheaves factors as inclusion into the presheaves followed by $\check{H}^{0}$. This leads to a spectral sequence we will call " $\breve{C}$ ech descent for sheaf cohomology":

$$
E_{2}^{p, q}=\check{H}^{p}\left(U / X ; H^{q}(-, A)\right) \Rightarrow H^{p+q}(X ; A)
$$

By passing to the limit in the spectral sequence above we get another spectral sequence

$$
E_{2}^{p, q}=\check{H}^{p}\left(X ; H^{q}(-, A)\right) \Rightarrow H^{p+q}(X ; A)
$$

Exercise: Study the case when $X=\operatorname{Spec} F, \mathrm{~F}$ a field. Show that the $\check{C}$ ech complex obtained from the nerve of a finite Galois extension $\mathrm{L} / \mathrm{F}$ is just the usual bar complex, the $\check{C}$ ech cohomology groups are exactly the groups labelled " $\check{C}$ ech" in section 1.2 , and the $\check{C}$ ech descent spectral sequence associated to $\mathrm{L} / \mathrm{F}$ is just the Hochshild-Serre spectral sequence of the group extension $G_{L} \rightarrow G_{F} \rightarrow G(L / F)$.

Now consider the question of whether $\check{C}$ ech and sheaf cohomology agree. This certainly isn't true in general, even on the site of a topological space. However from the $\check{C}$ ech descent spectral sequence we see that it would be 
enough to show that for a sheaf $\mathrm{A}, \check{H}^{p}\left(X ; H^{q}(-, A)\right)=0$ for $q>0$. The crudest way to guarantee these groups vanish would be to have some cofinal system of covers $\mathcal{U} \longrightarrow X$ such that $H^{q}\left(\mathcal{U}_{X}^{n} ; A\right)=0$ for all $\mathrm{U}, n$, and $q>0$. For example, this is how one shows that on the Zariski site of a separated noetherian scheme, sheaf and $\breve{C}$ ech cohomology agree for coherent sheaves ([18], III, 4.5). In this case one uses coverings by affine schemes, which are acyclic for coherent sheaf cohomology by a classical theorem of Serre.

This is far too much to expect in general, however. As a next attempt, one might start from the observation that on an arbitrary site, the sheafification of the presheaf $H^{q}(-, A)$ is zero when $q>0$. This amounts to the fact that for any $\mathrm{W}$ and $\alpha \in H^{q}(W ; A)$, there is a covering $\mathcal{V} \longrightarrow W$ such that $\alpha$ restricts to zero on $\mathcal{V}$. So what one needs to know is:

(*) For any covering $\mathcal{U} \longrightarrow X$ with $\check{C}$ ech nerve $\mathcal{U}_{X}$ and any covering $\mathcal{V} \longrightarrow \mathcal{U}_{X}^{n}$, there is a covering $\mathcal{U}^{\prime} \longrightarrow \mathcal{U}$ such that $\left(\mathcal{U}^{\prime}\right)_{X}^{n} \longrightarrow \mathcal{U}_{X}^{n}$ factors through $\mathcal{V}$.

Then we can conclude that every $\check{C}$ ech cochain on $\mathcal{U} / X$ with coefficients in $H^{q}(-, A)$ vanishes when we pass to the limit in $\mathcal{U}$. This is exactly what Artin [2] shows to get the following theorem (see also [42], 1.53).

Theorem 2.9 Let $X$ be any quasi-compact scheme such that every finite subset of $X$ lies in an open affine subscheme - for example, suppose $X$ is quasi-projective over a noetherian ring. Then for any additive presheaf $A$ on $X_{\text {ét }}$,

$$
H_{e t}^{*}(X ; a A) \cong \check{H}_{e t}^{*}(X ; A)
$$

This result is a special result about the étale site, and leaves open the question of whether there isn't a way to compute cohomology from covers more generally. An elegant answer to this question was provided by Verdier $([3], \mathrm{V}, 7)$. The idea is to replace the $\check{C}$ ech nerves by a more general simplicial covering object called a hypercover. The resulting cohomology theory, which we will call Verdier cohomology, agrees with sheaf cohomology on an arbitrary site - essentially because the appropriate analogue of $\left(^{*}\right)$ holds in general.

\subsection{Noetherian sites}

A site $\Phi$ is said to be noetherian if for every object $U$ and every covering family $\left\{U_{i} \longrightarrow U\right\}$, there is a finite subset $\left\{U_{i_{1}}, \ldots, U_{i_{n}}\right\}$ which is also a covering 
family. ${ }^{7}$ For example, if $\mathrm{X}$ is a noetherian scheme then the étale, Nisnevich and Zariski sites of $\mathrm{X}$ are noetherian (although in the case of the Nisnevich site this isn't obvious - see [44], appendix E, 6a). In fact it is enough to assume only that $\mathrm{X}$ is quasicompact, provided that the definition of étale morphism is modified as alluded to earlier (see [41],II,1.5.1).

Proposition 2.10 Let $\Phi$ be a noetherian site, $A_{i}$ a filtered (or even pseudofiltered) system of sheaves on $\Phi$. Then for all objects $U$, there is a natural isomorphism

$$
\operatorname{colim}_{i} H_{\Phi}^{*}\left(U ; A_{i}\right) \stackrel{\cong}{\longrightarrow} H_{\Phi}^{*}\left(U ; \operatorname{colim}_{i} A_{i}\right)
$$

To see why this is true, consider for the moment an arbitrary site, a presheaf $\mathrm{F}$ and a covering family $U_{i} \longrightarrow U$. Consider also the associated $\check{C}$ ech complex

$$
0 \longrightarrow F(U) \longrightarrow \prod_{i} F\left(U_{i}\right) \longrightarrow \prod_{i, j} F\left(U_{i} \times_{U} U_{j}\right) \longrightarrow \ldots
$$

Then by definition $\mathrm{F}$ is a sheaf if and only if for all such covering families of all objects $\mathrm{U}$, this complex is exact through the first three arrows, and is a flasque presheaf if and only if all such sequences are exact from the next term on (i.e. all the higher $\breve{C}$ ech cohomology groups vanish). We recall that injective sheaves are flasque, and that sheaf cohomology can be computed from flasque resolutions (see for example [41], I, 3.5). Thus the proof of the theorem boils down to showing that pseudo-filtered colimits take sheaves to sheaves, and flasque presheaves to flasque presheaves. This is clear provided that pseudo-filtered colimits commute with the products in the $\check{C}$ ech complex above. But if we now assume the site is noetherian, then we need only consider finite coverings, in which case all of these products are finite and the theorem follows.

The second important result of this section concerns the étale site of an inverse limit of schemes. Suppose $R_{i}$ is a filtered system of rings, and $R=\operatorname{colim}_{i} R_{i}$. Then $\operatorname{Spec} R$ is the (inverse) limit of the $R_{i}$ in the category of schemes. More generally, suppose $\mathcal{A}_{i}$ is a filtered system of quasicoherent $\mathcal{O}_{Y}$-algebras over a fixed base scheme $\mathrm{Y}$. Then one can define

\footnotetext{
${ }^{7}$ The reader might be wondering why we don't call this a quasicompact site. Note, however, that the definition generalizes the notion of a topological space $\mathrm{X}$ with the property that every open subset of $\mathrm{X}$ is quasicompact - that is, a noetherian topological space.
} 
associated schemes $\operatorname{Spec} \mathcal{A}_{i}$ (see [18],II,exercise 5.17), affine ${ }^{8}$ over Y, and $\operatorname{Spec}\left(\operatorname{colim}_{i} \mathcal{A}_{i}\right)$ is again the limit of the $\operatorname{Spec} \mathcal{A}_{i}$ in the category of schemes. Using this construction, it is not hard to show that if $X_{i}$ is any co-filtered system of schemes with affine transition maps $X_{i} \rightarrow X_{j}$, then the limit of the $X_{i}$ exists. However the only case we're really going to use is the affine case $X_{i}=\operatorname{Spec} R_{i}$, so the reader can feel free to picture this case, if desired.

Noetherian hypotheses are a bit awkward in this setting, because obviously a filtered colimit of noetherian rings need not be noetherian. For that reason we will work a bit more generally and assume we are given the following data:

- a cofiltered system of quasi-compact and quasi-separated ${ }^{9}$ schemes $X_{i}$, with affine transition maps. Such a system will be called admissible.

- an abelian presheaf $\mathrm{F}$ on a sufficiently large étale site (i.e. that includes all $X_{i}$ as well as $\mathrm{X}$, etc.). The presheaf $\mathrm{F}$ is assumed to be continuous in the sense that for any admissible system $U_{i}$ in the site, with limit $\mathrm{U}$, the natural map $\operatorname{colim}_{i} F\left(U_{i}\right) \longrightarrow F(U)$ is an isomorphism.

For example, any filtered system of rings, or of separated noetherian schemes with affine transition maps, defines an admissible system. There is no need to fuss over the precise meaning of "sufficiently large" in the second condition above, because in practice $\mathrm{F}$ will be a presheaf defined on all schemes -e.g. the K-theory presheaf, which is continuous as discussed in the next section. The following theorem is the Continuity Theorem.

Theorem 2.11 Let $X_{i}$ be an admissible system of schemes with limit $X$, and let $F$ be a continous presheaf on a sufficiently large étale site. Then there is a natural isomorphism

$$
\operatorname{colim}_{i} H_{e t}^{*}\left(X_{i} ; a F_{i}\right) \stackrel{\cong}{\longrightarrow} H_{e t}^{*}(X ; a F)
$$

\footnotetext{
${ }^{8} \mathrm{~A}$ morphism $f: X \longrightarrow Y$ is affine if $\mathrm{Y}$ has an open affine cover $U_{i}$ such that each $f^{-1} U_{i}$ is affine.

${ }^{9} \mathrm{~A}$ scheme is quasi-separated if the unique map to $S p e c \mathbb{Z}$ is quasicompact (i.e. the inverse image of every quasicompact open set is quasicompact). Every separated scheme is quasi-separated; see [16],I.6 for this and related issues.
} 
The proof of this important theorem is quite pretty, and as we will need one of the details, we will give a very brief sketch (see [1]). The beautiful fact is that essentially any morphism of schemes $f: U \rightarrow V$ over $\mathrm{X}$ is pulled back from a morphism $f_{i}: U_{i} \rightarrow V_{i}$ over some $X_{i}$, and moreover any reasonable property of $f$ can be arranged to hold for $f_{i}$. Given a scheme $\mathrm{U}$ over $\mathrm{X}$, we call $U_{i}$ over $X_{i}$ a source of $\mathrm{U}$ if $\mathrm{U}$ is isomorphic as $\mathrm{X}$-scheme to $U_{i} \times_{X_{i}} X$, and similarly for maps of X-schemes.

Lemma 2.12 Any étale $f: U \longrightarrow X$ has an étale source $U_{i} \longrightarrow X_{i}$, which can be taken surjective if $f$ is surjective. More generally, any morphism $U \rightarrow V$ of schemes étale over $X$ has a source $U_{i} \longrightarrow V_{i}$ with the same properties. Sources are essentially unique, in the sense that any two sources of $U \rightarrow V$ agree after base change to some higher $X_{j}$.

In fact by theorems of Grothendieck ([17],IV.8 and IV.11), the lemma holds with "étale" replaced by virtually any property $\mathrm{P}$ stable under base change - separated, finite, affine, projective, etc. The hardest step is obtaining a flat source.

Now let $\mathrm{G}$ be a presheaf on our big étale site. Ignoring the fact that $\mathrm{G}$ is already defined on $\mathrm{X}$, we define a presheaf $e G$ on $\mathrm{X}$ by

$$
e G=\operatorname{colim}_{i} \pi_{i}^{\#} G_{i}
$$

where $\pi_{i}^{\#}$ is the presheaf inverse image functor and $G_{i}$ is the restriction of $\mathrm{G}$ to $X_{i}$. Recall that the inverse image functor itself involves a gargantuan filtered colimit, so this definition appears to be a bit complicated. However it is easy to see on inspection:

Lemma 2.13 Let $U$ be étale over $X$, and let $U_{i}$ be any source of $U$. Then there is a natural isomorphism

$$
\operatorname{colim}_{j} G_{j}\left(U_{j}\right) \stackrel{\cong}{\longrightarrow}(e G)(U)
$$

where the colimit is over all $i \rightarrow j$ and $U_{j}$ is the base change of $U_{i}$ to $X_{j}$.

Note that if $\mathrm{G}$ is continuous, this shows that $e G$ is isomorphic to the restriction of $\mathrm{G}$ to $X_{e ́ t}$. Using this last lemma, and the fact that $X_{e ́ t}$ is a noetherian site, it is easy to show that the functor $e$ is exact, takes sheaves 
to sheaves, and takes flasque presheaves to flasque presheaves. The argument is just as in Theorem 2.10, and the proof of Theorem 2.11 is then completed the same way.

Here is the sort of application we have in mind. Let $\mathrm{X}$ be a scheme, $\mathrm{F}$ a sheaf on $X_{\text {ét }}$.

Corollary 2.14 Consider the presheaf $U \mapsto H_{e t}^{*}(U ; F)$ on the Zariski, Nisnevich and étale sites of $X$. Then

(i) The Zariski stalk at $x \in X$ is $H_{\text {ét }}^{*}\left(\operatorname{Spec} \mathcal{O}_{x} ; e F\right)$

(ii) The Nisnevich stalk at $x^{\prime} \rightarrow x$ is $H_{e t}^{*}\left(\operatorname{Spec} \mathcal{O}_{x^{\prime}}^{h} ; e F\right)$

(iii) The étale stalk at $\bar{x} \rightarrow x$ is $H_{e t}^{*}\left(\operatorname{Spec} \mathcal{O}_{\bar{x}}^{s h} ; e F\right)(=0$ for $*>0)$.

Note we are not assuming $\mathrm{F}$ is continuous, or even defined on the various local rings, in the above corollary. If it happens to be continuous, that's fine, but the corollary as stated is needed for example in Theorem 2.8. Its proof is easy from the continuity theorem and the definition of stalks; recall that in all three cases these can be computed from a cofiltered limit of affine schemes. Of course the fact that the étale stalk is zero follows from more elementary considerations.

\section{Hypercohomology spectra on schemes}

In this section we introduce and compare the cohomological descent machines of Thomason ([42], see especially sections 1 and 5) and Jardine ([20], [21], $[22])$.

We begin with some homotopy-theoretic preliminaries, including a few words on closed model categories and our conventions on spectral sequences. It is a basic fact of life that homotopy limits (and various other constructions) do not commute with filtered colimits. Nevertheless, there are many situations where a filtered colimit can be "commuted" with whatever other construction is at hand. The "colimit lemma" (3.3) is an explicit and practical criterion for deciding the issue; it will be used frequently in the sequel.

Next we introduce Thomason's spectrum-level Godement construction. This is the analogue, for presheaves of spectra, of the hypercohomology of a chain complex of sheaves. (The reader is urged to consult Thomason's 
"Scholium of Great Enlightenment" at this point ${ }^{10}([42], 5.32)$.) It can also be regarded as a generalization of the homotopy fixed point set discussed in section 1. Virtually any of the standard theorems on abelian sheaf cohomology can be extended to hypercohomology spectra - although typically some boundedness or finite cohomological dimension hypotheses must be imposed. Two important examples are given, including the spectrum-level continuity theorem. Further examples can be found in [42],1. We also give conditions for commuting smash products and Bousfield localization with hypercohomology.

It should be noted that the Thomason-Godement construction, as given here, only applies to sites with enough points, although according to Thomason $([42], 1.34)$, there is a modified version that works in the general case.

The next section surveys the elegant work of Jardine [20] [21] that establishes a closed model category structure on presheaves of spectra. In this approach, Thomason's hypercohomology spectrum $\mathbb{H}^{\cdot}(-; \mathcal{E})$ is replaced by a globally fibrant model for $\mathcal{E}$. It is not as obvious in this setting that there is a descent spectral sequence, so we explain this point in more detail. Finally, we compare the globally fibrant model with $\mathbb{H}^{\cdot}(-; \mathcal{E}$ ) (on a site with enough points). The conclusion is that, subject to a finite cohomological dimension assumption, the two constructions agree.

In the last section we give a brief introduction to $\check{C}$ ech hypercohomology spectra $([42], 1)$. Once again, results from abelian sheaf theory generalize to the spectrum setting without much difficulty - for example, "Č ech descent for sheaf cohomology", and Artin's theorem [2] relating Čech and sheaf cohomology on the étale site.

Throughout this work, the adjective pointwise always indicates that a property of presheaves or morphisms of presheaves holds for the sections on any object $U$ of the site. For example a map $\mathcal{E} \longrightarrow \mathcal{F}$ of presheaves of spectra is a pointwise weak equivalence if each map $\mathcal{E}(U) \longrightarrow \mathcal{F}(U)$ is a weak equivalence, and so on.

\footnotetext{
${ }^{10}$ The referee points out that the "scholium" is not as enlightening as it could be, because the assertions found there are neither proved nor obvious. For proofs, see Chapter 4 of [22].
} 


\subsection{Preliminaries on homotopy theory}

\subsubsection{Spectral sequences}

In this section we explain our conventions on spectral sequences. For a very complete analysis of convergence see [4], where the term "conditionally convergent" is coined.

Suppose given a tower of fibrations of spectra

$$
* \stackrel{f_{0}}{\longleftarrow} X_{0} \stackrel{f_{1}}{\longleftarrow} X_{1} \stackrel{f_{2}}{\longleftarrow} \ldots
$$

and let $W_{n}$ denote the homotopy-fibre of $f_{n}$. Applying $\pi_{*}$ yields an exact couple and a spectral sequence which we index by setting

$$
E_{1}^{p,-q}=\pi_{q-p} W_{p}
$$

This is a right half-plane cohomology spectral sequence, with the "AtiyahHirzebruch" style of indexing (motivated by the example in which $X_{n}$ is the function spectrum $F\left(A_{n}, E\right)$ for some spectrum $\mathrm{E}$ and $\mathrm{CW}$-complex A with skeletal filtration $A_{n}$ ).

Let $F^{p}=F^{p} \pi_{*} X$ denote the kernel of the projection map $\pi_{*} X \longrightarrow \pi_{*} X_{p-1}$. The spectral sequence converges if (i) the natural inclusion $F^{p} / F^{p+1} \subset E_{\infty}^{p, *}$ is an isomorphism for all $p$; and (ii) $\lim _{n}^{1} \pi_{*} X_{n}=0$. In fact the spectral sequence is always conditionally convergent - that is, it converges if and only if $\lim _{r}^{1} E_{r}^{p, q}=0$. Thus the spectral sequence automatically converges if the $E_{2}$-term is bounded on the right, bounded below, or is finite in each bidegree.

For example, suppose I is a small category and $i \longrightarrow X_{i}$ a functor to spectra. Then the methods of [6],XI, lead to a tower of fibrations and a spectral sequence

$$
E_{2}^{p,-q}=\lim _{i}^{p} \pi_{q} X_{i} \Rightarrow \pi_{q-p} \text { holim }_{i} X_{i}
$$

where $\lim ^{p}$ is the $p$-th derived functor of the inverse limit. If $I=\Delta$, so that $X^{\cdot}$ is a cosimplicial spectrum, we have $E_{2}^{p,-q}=\pi^{p}\left(\pi_{q} X^{\cdot}\right)$, where $\pi^{p}$ denotes the cohomotopy of a cosimplicial abelian group - that is, the cohomology of the associated chain complex ([6],XI,7.3).

Finally, we remark that we could also consider an extended tower

$$
\ldots \longleftarrow X_{-1} \longleftarrow X_{0} \longleftarrow X_{1} \longleftarrow \ldots
$$


such as the Postnikov tower of a non-connective spectrum. This leads to a similar spectral sequence, but now the spectral sequence is no longer confined to a half-plane and the convergence question becomes more delicate. Nevertheless, in some cases it is still conditionally convergent. Suppose, for example, that $\pi_{k} X_{n}=0$ for $k>n$. Then it is even possible to re-index the spectral sequence so that it becomes a right half-plane spectral sequence - compare [42], p. 542. See Theorem 3.19 below for an example of this situation.

\subsubsection{Closed model categories}

A closed model category is a category $\mathcal{C}$ equipped with three distinguished classes of morphisms, called weak equivalences, fibrations and cofibrations, that are closed under composition and contain all identities. These classes are subject to several axioms that codify the abstract properties of a "homotopy theory".

(CM1): $\mathcal{C}$ has all finite limits and colimits.

(CM2): If $f=g h$ is a composite morphism and any two of $f, g, h$ are weak equivalences, then so is the third.

(CM3): All three classes of morphisms are closed under retracts.

(CM4): Given a commutative square

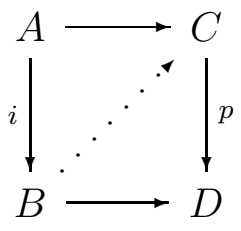

the dotted arrow exists whenever (i) $i$ is an acyclic cofibration and $p$ is a fibration, or (ii) $i$ is a cofibration and $p$ is an acyclic fibration ("acyclic" is shorthand for weak equivalence).

(CM5): Any map $X \rightarrow Y$ can be factored in two ways: (i) as $X \stackrel{i}{\longrightarrow} W \stackrel{p}{\longrightarrow} Y$, where $i$ is a cofibration and $p$ is an acyclic fibration; and (ii) as $X \stackrel{j}{\longrightarrow} Z \stackrel{q}{\longrightarrow}$ $Y$, where $j$ is an acylic cofibration and $p$ is a fibration. 
Let $\phi$ and $*$ denote respectively "the" initial and terminal objects of the category (usually, for us, $\phi=*$ ). An object $\mathrm{X}$ is cofibrant if $\phi \rightarrow X$ is a cofibration and fibrant if $X \rightarrow *$ is a fibration. Part of the general philosophy is that homotopy theory works best with a cofibrant source and a fibrant target (see [11],3.18 and 3.22). In practice one of these two conditions holds trivially for all - or almost all - objects of the category, while the other imposes possibly stringent conditions on an object X. For example, in the category of presheaves of spectra discussed below, cofibrancy is a harmless condition to impose (although not all objects are cofibrant), whereas fibrancy or the lack of it is essentially the descent problem.

The most basic example for present purposes is the category of pointed simplicial sets. We say that a map of pointed simplicial sets is a weak equivalence if the induced map on realizations is a weak equivalence in the usual topological sense, is a cofibration if it is injective, and is a fibration if it is a Kan fibration. With these definitions, pointed simplicial sets form a closed model category [36]; of course this works in the unpointed category also. Every object is cofibrant, and the fibrant objects are the Kan complexes.

This closed model category structure was extended to spectra in [5]. Here a spectrum is defined to be a sequence of pointed simplicial sets $X_{n}, n \geq 0$, equipped with structure maps $S^{1} \wedge X_{n} \rightarrow X_{n+1}$. The homotopy groups of a spectrum $\mathrm{X}$ are defined as usual by

$$
\pi_{n} X=\operatorname{colim}_{k} \pi_{n+k}\left|X_{n+k}\right|
$$

with the colimit taken in the evident way using the structure maps. We say that a map of spectra $f: X \rightarrow Y$ is a weak equivalence if it induces an isomorphism on homotopy groups, and is a cofibration if for all $n \geq 0$ the natural map from the pushout $P_{n}$ in the diagram

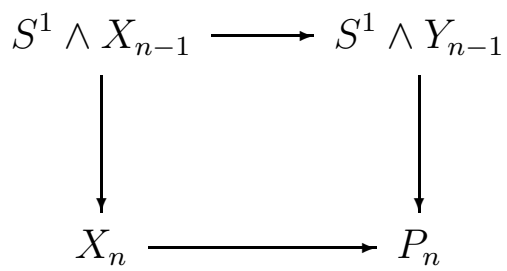

to $Y_{n}$ is a cofibration of simplicial sets (in particular $f$ is a spacewise cofibration). Finally, we say that $f$ is a fibration if it has the right lifting property 
as in CM4(ii) with respect to all acyclic cofibrations. With these definitions, the category of spectra is a closed model category [5]. The fibrations are in particular spacewise fibrations, and can be described explicitly (see [5]). It turns out that the fibrant spectra are precisely the $\Omega$-spectra (in the classical sense) whose constituent spaces are Kan complexes. The cofibrant spectra are just the spectra with injective structure maps.

\subsubsection{Filtered colimits of spectra}

Consider the closed model category of pointed simplicial sets, as defined above. Then:

Proposition 3.1 Filtered colimits preserve cofibrations and fibrations (in particular a filtered colimit of Kan complexes is a Kan complex). Filtered colimits of Kan complexes preserve weak equivalences.

For cofibrations, this is obvious, since a cofibration is just an injective map. The proof for fibrations may be sketched as follows. Call a simplicial set $\mathrm{K}$ small if it has only finitely many nondegenerate simplices. Then it is easy to see that the functor $\operatorname{Hom}(K,-)$ commutes with filtered colimits (even when the maps in the colimit are not injective!). But Kan fibrations are defined in terms of the right lifting property with respect to certain maps of small simplicial sets (inclusion of the boundary minus one face of $\Delta^{n}$ into $\Delta^{n}$ ), and the result follows. A similar argument applies to weak equivalences, provided that the topological homotopy groups occurring in the definition of weak equivalence can be replaced by simplicial homotopy groups. This is possible since we have assumed the spaces in question are Kan complexes.

Similar arguments apply to the Bousfield-Friedlander closed model category structure on spectra, although the situation is complicated slightly by the different notion of fibration. Call a spectrum X a Kan spectrum if each space $X_{n}$ is a Kan complex. The result is:

Proposition 3.2 Filtered colimits of spectra preserve cofibrations and fibrations, and filtered colimits of Kan spectra preserve weak equivalences.

In fact the assertions for cofibrations and weak equivalences hold for pseudofiltered colimits. We note that up to functorial weak equivalence, 
any spectrum can be replaced by a Kan spectrum; this will be done tacitly, when necessary, in the sequel.

We now turn to the (critical!) question of when homotopy limits and related constructions commute with filtered colimits. Our goal is to give a simple criterion that is easily applied in practice - although it will take longer to state this criterion than to prove it. Throughout this section "spectral sequence" means right half-plane cohomology spectral sequence.

We assume given the following data:

- A spectrum $\mathrm{X}$ and a filtered system of spectra $X_{i}, i \in I$.

- A compatible family of maps $f_{i}: X_{i} \longrightarrow X$, so there is an induced map $\operatorname{colim} X_{i} \longrightarrow X$.

- Spectral sequences $E_{*} X_{i}$ and $E_{*} X$ converging conditionally to $\pi_{*} X_{i}$ and $\pi_{*} X$ respectively, and that are natural with respect to the maps in the filtered system and with respect to the $f_{i}$. (In particular the associated filtrations on $\pi_{*} X_{i}$ and $\pi_{*} X$ are assumed natural in this sense.)

We say that a spectral sequence is bounded on the right if there exists $d$ such that $E_{2}^{p, *}=0$ for $p>d$, and is bounded below if there exists $m$ such that $E_{2} *, q=0$ for $q<m$. Note that either condition implies convergence. If we are given a family $E_{*}(i)$ of spectral sequences, we say that $E_{*}(i)$ is uniformly bounded on the right if there is a fixed $d$ that works for all $i$, etc. The next proposition will be called the "colimit lemma".

Proposition 3.3 Suppose that (i) either the $E_{2}$-terms $E_{2}\left(X_{i}\right)$ are uniformly bounded on the right, or else they are uniformly bounded below; and (ii) the map $f_{*}:$ colim $_{i} E_{2}\left(X_{i}\right) \longrightarrow E_{2}(X)$ is an isomorphism. Then the map $f: \operatorname{colim}_{i} X_{i} \longrightarrow X$ is a weak equivalence.

Proof: Since the index category I is filtered, the colimit of the spectral sequences $E_{*}(i)$ is still a spectral sequence. However it need not, in general, converge to the homotopy of the colimit - the trouble is that nontrivial elements of $\pi_{*} \operatorname{colim}_{i} X_{i}=\operatorname{colim}_{i} \pi_{*} X_{i}$ can have infinite filtration. But hypothesis (i) rules out this possibility; the induced filtration on $\operatorname{colim}_{i} \pi_{q} X_{i}$ is finite for each $q$, and the colimit of the spectral sequences converges to the homotopy of the colimit. Then condition (ii) implies that the target 
spectral sequence is also convergent, the induced map on $E_{\infty}$-terms is an isomorphism, and $f$ is a weak equivalence.

As an illustration, suppose that I and $J$ are small categories with I filtered and $\mathrm{J}$ arbitrary. Let $X_{i, j}$ be an $(I \times J)$-diagram of spectra, and consider the natural map

$$
f: \operatorname{colim}_{i} \text { holim }_{j} X_{i, j} \longrightarrow \text { holim }_{j} \operatorname{colim}_{i} X_{i, j}
$$

Here "holim" means the homotopy limit as in [6], extended to spectra as in [42], section 5. For present purposes, all the reader needs to know is that (1) holim is a functor from J-diagrams $Y_{j}$ of spectra to spectra; and (2) there is a natural conditionally convergent spectral sequence

$$
E_{2}^{p,-q}=\lim _{j}^{p}\left(\pi_{q} Y_{j}\right) \Rightarrow \pi_{q-p} h o l i m Y_{j}
$$

where $\lim ^{p}$ denotes the p-th derived functor of the inverse limit. Recall that a spectrum $\mathrm{X}$ is bounded above if its homotopy groups vanish above some dimension. Then we obtain as an immediate corollary:

Proposition 3.4 Suppose that (i) either there exists d such that for all $q, i$ the groups $\lim _{j}^{p}\left(\pi_{q} X_{i, j}\right)$ vanish for $p>d$, or else the $X_{i, j}$ are uniformly bounded above; and (ii) the natural map

$$
\operatorname{colim}_{i} \lim _{j}^{p} \pi_{*} X_{i, j} \longrightarrow \lim _{j}^{p} \operatorname{colim}_{i} \pi_{*} X_{i, j}
$$

is an isomorphism. Then the map

$$
f: \operatorname{colim}_{i} \operatorname{holim}_{j} X_{i, j} \longrightarrow \text { holim }_{j} \operatorname{colim}_{i} X_{i, j}
$$

is a weak equivalence.

For example if $J=\Delta$, so we have a filtered system of cosimplicial spectra, condition (ii) holds automatically but condition (i) is a nontrivial assumption about finite cohomological dimension. On the other hand if $\mathrm{J}$ is the category $0 \leftarrow 1 \leftarrow 2 \leftarrow \ldots$, so a J-diagram is a tower, then condition (i) holds with $d=1$ but condition (ii) can easily fail. Let's call a tower of spectra $Y_{j}$ stable if for each fixed $q$ there is an $m_{q}$ such that $\pi_{q} Y_{j} \rightarrow \pi_{q} Y_{j-1}$ is an isomorphism for all $n>m_{q}$ (for example, a Postnikov tower). We call an I-diagram of towers $X_{i j}$ as above uniformly stable if each tower $X_{i j}(i$ fixed) is stable and 
moreover each number $m_{q}$ depends only on $q$ and not on $i$. In this case $\pi_{q} \lim _{j} X_{i j}=\pi_{q} X_{i, n}$ with $n>>0$ depending only on $q$. Then (ii) obviously holds and we get the easy but important corollary:

Corollary 3.5 Let $X_{i j}$ be a filtered system (in i) of towers (in $j$ ), and suppose the system is uniformly stable. Then

$$
\operatorname{colim}_{i} \lim _{j} X_{i, j} \stackrel{\cong}{\longrightarrow} \lim _{j} \operatorname{colim}_{i} X_{i, j}
$$

Remark 3.6 The assumption on $E_{2}$-terms in the colimit lemma can, of course, be replaced by the same assumption on some other fixed $E_{r}$-term. An interesting example is discussed in the introduction to Section 4.

\subsection{The Godement construction for spectra}

Let $\Phi$ be a site with enough points - for example, the Zariski, étale, or Nisnevich site of a scheme X. Let $\mathcal{E}$ be a presheaf of spectra on $\Phi$. Then the construction of the Godement resolution for ordinary abelian sheaves carries over almost verbatim to presheaves of spectra. In fact let $\mathcal{A}$ be any category with filtered colimits, products and coproducts, and consider an $\mathcal{A}$-valued presheaf $\mathrm{F}$. Then we can define the stalk $F_{p}=p^{*} F$ at a point $p$ as a filtered colimit over neighborhoods of $p$ as in 2.6. The formula given there for the right adjoint $p_{*}$ works fine too. The Godement construction then produces a cosimplicial $\mathcal{A}$-valued presheaf $T \cdot F$, whose sections on $\mathrm{X}$ give a cosimplicial object in $\mathcal{A}$. In particular we get a Godement functor $\mathcal{E} \longrightarrow T \cdot \mathcal{E}$ from presheaves of spectra to cosimplicial presheaves of spectra, and we define the hypercohomology of $\mathrm{X}$ with coefficients in $\mathcal{E}$ by

$$
\mathbb{H}_{\Phi}(X ; \mathcal{E})=\text { holim }_{\Delta} T \cdot \mathcal{E}(X)
$$

In fact $\mathbb{H}_{\Phi}^{;}(-; \mathcal{E})$ is itself a presheaf on the site. Note that $\pi_{*} T \cdot \mathcal{E}=T \cdot \pi_{*} \mathcal{E}$. Hence the Bousfield-Kan machinery discussed above yields a conditionally convergent spectral sequence

$$
E_{2}^{p,-q}=H_{\Phi}^{p}\left(X ; \tilde{\pi}_{q} \mathcal{E}\right) \Rightarrow \pi_{q-p} \mathbb{H}_{\Phi}^{\prime}(X ; \mathcal{E})
$$

Many basic results about cohomology can now be extended rather easily to hypercohomology spectra. We illustrate this with some examples that will 
be important later. Given a site $\Phi$, a presheaf of spectra $\mathcal{E}$ and an object $\mathrm{X}$ of the site, we say that $c d^{\Phi}(X ; \mathcal{E})$ is bounded, or $c d \Phi<\infty$, if for some $d$ $H^{p}\left(X ; \tilde{\pi}_{q} \mathcal{E}\right)=0$ for all $p>d$ and all $q$. If the presheaf and/or the object are varying over a family, the phrase "uniformly bounded" means that a fixed $d$ works for all presheaves and/or objects in the family. A condition is said to hold stalkwise if it holds for the stalks of the presheaf in question. Often this makes sense even without reference to points of the site - for example, a presheaf of spectra is stalkwise bounded above if for some $m$ the sheaves $\tilde{\pi}_{q}$ vanish for all $q>m$.

Theorem 3.7 Suppose that $\Phi$ is a noetherian site, $\mathcal{E}_{i}$ a pseudofiltered system of presheaves of spectra on $\Phi$. Fix an object $X$ of $\Phi$ and suppose that either (a) $c d^{\Phi}\left(X ; \mathcal{E}_{i}\right)$ is uniformly bounded, or (b) the $\mathcal{E}_{i}$ are stalkwise uniformly bounded above. Then the natural map

$$
\operatorname{colim}_{i} \mathbb{H}^{\cdot}\left(X ; \mathcal{E}_{i}\right) \longrightarrow \mathbb{H}_{\Phi}^{*}\left(X ; \operatorname{colim}_{i} \mathcal{E}_{i}\right)
$$

is a weak equivalence.

Proof: One simply checks the conditions of the colimit lemma 3.3. Condition (i) of that lemma holds by assumption, and condition (ii) is immediate from the algebraic version 2.10 .

This result is important because it will allow us to commute smash products and Bousfield localizations with hypercohomology (see the end of this section).

Similarly, we can promote the continuity theorem 2.11 to the spectrum level. Thus suppose $X_{i}$ is an admissible system of schemes (i.e. a cofiltered system of quasicompact and quasiseparated schemes, with affine transition maps), with $X=\lim _{i} X_{i}$. Recall that the main case to keep in mind is that of a filtered system of rings, such as the system used to define henselizations. Let $\mathcal{E}$ be a presheaf of $\ell$-torsion spectra on a sufficiently large étale site, and suppose that $\mathcal{E}$ is continuous in the sense that for any admissible system $U_{i}$ in the site, the natural map $\operatorname{colim}_{i} \mathcal{E}\left(U_{i}\right) \longrightarrow \mathcal{E}(U)$ is a weak equivalence.

Theorem 3.8 Suppose that $X_{i}$ is an admissible system of schemes, $\mathcal{E}$ a continuous presheaf of $\ell$-torsion spectra as above. Suppose also that either (a) $c d^{\Phi}\left(X_{i} ; \mathcal{E}\right)$ is uniformly bounded, or $(b) \mathcal{E}$ is stalkwise bounded above. Then the natural map 


$$
\operatorname{colim}_{i} \mathbb{H}^{\prime}{ }^{\prime}\left(X_{i} ; \mathcal{E}\right) \longrightarrow \mathbb{H}^{\prime}{ }^{\prime}(X ; \mathcal{E})
$$

is a weak equivalence.

Again, the proof is immediate from the colimit lemma together with the continuity theorem 2.11. The one point to note is that the presheaves $\pi_{q} \mathcal{E}$ are continuous by assumption, and hence so are the associated sheaves $\tilde{\pi}_{q} \mathcal{E}$.

The main example of a continuous presheaf of spectra is the K-theory presheaf. It is continuous by a theorem of Quillen ([35], 7,2.2). The point is that the category of locally free sheaves on the limit scheme $\mathrm{X}$ is the "colimit" of the corresponding categories on the $X_{i}$.

Our main application of continuity is to the determination of the stalks of hypercohomology presheaves.

Theorem 3.9 Let $X$ be a separated noetherian scheme of finite Krull dimension. Suppose $X$ is uniformly $\ell$-bounded. Let $\mathcal{E}$ be a presheaf of $\ell$-torsion spectra on $X_{e ́ t}$ and regard $\mathcal{F}=\mathbb{H}^{\prime} e t(-; \mathcal{E})$ as a presheaf on $X_{Z a r}, X_{N i s}$, or $X_{\text {ét }}$. Then

(i) The Zariski stalk of $\mathcal{F}$ at $x$ is $\mathbb{H}^{\prime}{ }^{\prime} t\left(\mathcal{O}_{x} ; \mathcal{E}\right)$

(ii) The Nisnevich stalk of $\mathcal{F}$ at $x^{\prime} \rightarrow x$ is $\mathbb{H}^{\prime}{ }^{\prime} t\left(\mathcal{O}_{x^{\prime}}^{h} ; \mathcal{E}\right)$

(iii) The étale stalk of $\mathcal{F}$ at $\bar{x} \rightarrow x$ is $\mathbb{H}^{\prime} e^{e} t\left(\mathcal{O}_{\bar{x}}^{s h} ; \mathcal{E}\right)$

Proof: Consider for example case (iii), where we can work on the restricted étale site. If $U=\operatorname{Spec} \mathcal{O}_{\bar{x}}^{s h}$, then $U=\lim _{i} U_{i}$, where $U_{i}$ ranges over the cofiltered system of étale neighborhoods of $\bar{x}$. The $U_{i}$ can be taken affine and hence form an admissible system. Moreover the assumptions on $\mathrm{X}$ are inherited by the $U_{i}$, so that $c d_{\ell}^{e t}\left(U_{i}\right)$ is bounded uniformly in $i$. Hence Theorem 3.8 applies, completing the proof.

Now suppose $\mathrm{W}$ is a fixed spectrum. Then for any presheaf of spectra $\mathcal{E}$ there is a natural map

$$
W \wedge \mathbb{H}^{\prime}(X ; \mathcal{E}) \longrightarrow \mathbb{H}_{\Phi}{ }_{\Phi}(X ; W \wedge \mathcal{E})
$$

which in general need not be a weak equivalence. However smashing with a finite spectrum commutes with arbitary homotopy limits and products, hence: 
Proposition 3.10 If $W$ is a finite spectrum, then for any presheaf of spectra $\mathcal{E}$ and object $X$ the map

$$
W \wedge \mathbb{H}^{\circ}{ }_{\Phi}(X ; \mathcal{E}) \longrightarrow \mathbb{H}^{\cdot}(X ; W \wedge \mathcal{E})
$$

is a weak equivalence.

If $\mathrm{W}$ is not finite then some condition has to be imposed on the site, the object, the presheaf or all three. The next result is not the most general one can imagine, but takes the form that we will typically need.

Proposition 3.11 Suppose $c d_{\ell}^{\Phi}(X)<\infty$, and that either $W$ is an $\ell$-torsion spectrum or $\mathcal{E}$ is a presheaf of $\ell$-torsion spectra. Then the map

$$
W \wedge \mathbb{H}^{*}(X ; \mathcal{E}) \longrightarrow \mathbb{H}^{\cdot}{ }_{\Phi}(X ; W \wedge \mathcal{E})
$$

is a weak equivalence.

Proof: $\mathrm{W}$ is a directed colimit of finite spectra $W_{i}$, which can be taken $\ell$-torsion if $\mathrm{W}$ is. Then

$$
W \wedge \mathbb{H}_{\Phi}^{\circ}(X ; \mathcal{E})=\operatorname{colim}_{i} W_{i} \wedge \mathbb{H}^{\circ}(X ; \mathcal{E})=\operatorname{colim}_{i} \mathbb{H}^{\circ}{ }_{\Phi}\left(X ; W_{i} \wedge \mathcal{E}\right)
$$

by the preceeding proposition. Now each presheaf $W_{i} \wedge \mathcal{E}$ is a presheaf of $\ell$-torsion spectra, so Theorem 3.7 applies.

Finally, recall that a spectrum $\mathrm{E}$ is smashing if $L_{E} Z=Z \wedge L_{E} S^{0}$ for all $Z$, or equivalently $L_{E}$ commutes with directed colimits. For example the complex K-theory spectrum is smashing, but the mod $\ell$ Moore spectrum is not (completion doesn't commute with direct limits).

Corollary 3.12 Suppose $c d_{\ell}^{\Phi}(X)<\infty, E$ is smashing, and that either $L_{E} S^{0}$ is an $\ell$-torsion spectrum or $\mathcal{E}$ is a presheaf of $\ell$-torsion spectra. Then the natural map

$$
L_{E} \mathbb{H}^{\circ} \Phi(X ; \mathcal{E}) \longrightarrow \mathbb{H}^{\circ}\left(X ; L_{E} \mathcal{E}\right)
$$

is a weak equivalence. 


\subsection{Jardine's closed model category}

Let $\Phi$ be a site and consider the category $\mathcal{P}$ of presheaves of spectra on $\Phi$. We would like to define a closed model category structure on $\mathcal{P}$ in a way that depends on the Grothendieck topology, not just the underlying category. In particular the weak equivalences should depend on the topology, and the fibrant objects should be the objects satisfying descent.

The most natural definition of weak equivalence is stalkwise weak equivalence. We say that a map of presheaves $\mathcal{E} \longrightarrow \mathcal{F}$ is a stalkwise weak equivalence if the induced map of associated abelian sheaves $\tilde{\pi}_{*} \mathcal{E} \longrightarrow \tilde{\pi}_{*} \mathcal{F}$ is an isomorphism. Note this definition makes sense even when $\Phi$ doesn't have enough points. If $\Phi$ does have enough points, as is the case in all the examples we care about, then a map is a stalkwise weak equivalence if and only if the induced maps on stalks are weak equivalences of spectra. However even in this case it will be convenient to have available the definition as stated. Note that every pointwise weak equivalence is a stalkwise weak equivalence, since pointwise weak equivalence just means that the induced map on homotopy presheaves is already an isomorphism. The converse is obviously false.

Remark. Any stalkwise weak equivalence is also a stalkwise homology isomorphism. This is clear for a site with enough points, but not at all obvious in the general case. See [22], 2.9.

A map of presheaves is a cofibration if it is a pointwise cofibration, and is a fibration if it has the right lifting property with respect to cofibrations that are also stalkwise weak equivalences. The following theorem is due to Jardine [21]. Earlier results of this type, but for sheaves of simplicial sets, were proved Brown-Gersten [7] in the Zariski case and Joyal in the general case.

Theorem 3.13 Let $\Phi$ be an arbitrary site. Then with the above definitions, the category $\mathcal{P}$ of presheaves of spectra on $\Phi$ is a closed model category.

In fact Jardine shows that $\mathcal{P}$ is a proper simplicial model category. The proof of Theorem 3.13 is discussed at the end of this section.

In order to avoid possible confusion with either pointwise fibrations or the local fibrations of [20], the fibrations in this closed model category structure

are called global fibrations. Similarly a presheaf is globally fibrant if it is 
fibrant in the closed model category structure of Theorem 3.13. Note that the cofibrant objects are just the presheaves which are pointwise cofibrant in the sense of Bousfield-Friedlander. In particular any presheaf is pointwise weak equivalent to a cofibrant presheaf, so there is no harm in assuming that our presheaves are cofibrant.

Now by CM5, given any presheaf $\mathcal{E}$ we can find a globally fibrant presheaf $G \mathcal{E}$ and a map $\mathcal{E} \longrightarrow G \mathcal{E}$ which is both a cofibration and a stalkwise weak equivalence. It follows from the proof of Theorem 3.13 that $G \mathcal{E}$ can be chosen functorially. It is convenient, however, not to be tied down to any particular choice; any such stalkwise weak equivalence (whether or not it is a cofibration) will be called a globally fibrant model for $\mathcal{E}$. This globally fibrant model (which we will see shortly is well-defined up to pointwise weak equivalence) will serve as an alternative to, and generalization of, Thomason's hypercohomology spectrum. Before justifying this assertion, we summarize a few properties of Jardine's closed model category structure, properties which begin to illustrate the elegance of this approach. We first consider the behaviour of the three classes of morphisms under direct image, inverse image, and extension by zero.

Proposition 3.14 Let $f: \Phi \longrightarrow \Phi^{\prime}$ be a site morphism. Then the presheaf inverse image functor $f^{\#}$ preserves cofibrations and stalkwise weak equivalences, and the direct image functor $f_{\#}$ preserves cofibrations and global fibrations.

The proof of the first statement is easy, using the fact that $f^{\#}$ is defined in terms of pseudofiltered colimits of spectra. Obviously $f_{\#}$ preserves cofibrations. That $f_{\#}$ preserves global fibrations is a formal consequence of the fact that it has a left adjoint, namely $f^{\#}$, that preserves cofibrations and weak equivalences. It is clear that $f^{\#}$ does not generally preserve global fibrations, and $f_{\#}$ does not generally preserve stalkwise weak equivalences (consider for example a scheme with the Zariski topology and a map to Spec of a field).

Here is a simple example that will be used later. Consider a scheme $\mathrm{X}$ and a presheaf $\mathcal{E}$ which is globally fibrant on the étale site. Applying the proposition to the restriction morphisms $X_{\text {ét }} \longrightarrow X_{N i s} \longrightarrow X_{Z a r}$ shows that $\mathcal{E}$ is globally fibrant on the Nisnevich and Zariski sites as well. 
Corollary 3.15 Let $j: \Phi / U \longrightarrow \Phi$ denote inclusion of a local site as in section 2.5. Then the extension by zero functor $j_{\text {! }}$ preserves cofibrations and stalkwise weak equivalences, and $j^{\#}$ preserves global fibrations.

This follows immediately because $j^{\#}=k_{\#}$ and $j_{!}=k^{\#}$, where $k$ is as in section 2.5. We also deduce:

Corollary 3.16 Every global fibration is a pointwise fibration.

For suppose given a global fibration $\pi: \mathcal{E} \longrightarrow \mathcal{F}$. We wish to show that for every object $\mathrm{U}$ of the site, $\mathcal{E}(U) \longrightarrow \mathcal{F}(U)$ is a fibration of spectra. But the "sections on U" functor has a left adjoint that preserves cofibrations and stalkwise weak equivalences - namely, the functor $E \mapsto j_{!} c_{E}$, where $c_{E}$ is constant presheaf on $\Phi / U$ associated to the spectrum E.

As noted above, a stalkwise weak equivalence need not be a pointwise weak equivalence. There is one case, however, where we do get pointwise weak equivalence. I thank Paul Goerss and the referee for providing the proof of the following important proposition.

Proposition 3.17 Suppose $f: \mathcal{E} \longrightarrow \mathcal{F}$ is a stalkwise weak equivalence of globally fibrant presheaves. Then $f$ is a pointwise weak equivalence.

Using CM5 and CM2, we reduce immediately to two cases: either $f$ is a global fibration, or $f$ is a cofibration. If $f$ is a global fibration, the adjoint functor argument used above shows that $f$ is a pointwise weak equivalence. If $f$ is a cofibration, then $f$ is a homotopy equivalence - in fact, $\mathcal{E}$ is a deformation retract of $\mathcal{F}$. To explain this assertion, we need a tiny bit of the "simplicial" model category structure on presheaves of spectra. If $\mathcal{A}$ is any presheaf of spectra, let $\mathcal{A}^{\Delta^{1}}$ denote the path object of simplicial maps $\Delta^{1} \longrightarrow \mathcal{A}$ (defined pointwise and levelwise). Homotopies can then be defined using this path object, in the usual way. Now since $\mathcal{E}$ is globally fibrant, there is a lift $r$ in the diagram

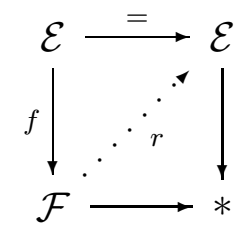


so that $r f=1_{\mathcal{E}}$. On the other hand, since $\mathcal{F}$ is globally fibrant, the obvious map $\mathcal{F}^{\Delta^{1}} \longrightarrow \mathcal{F} \times \mathcal{F}$ is a global fibration (this is a simple special case of Quillen's axiom "SM7" for simplicial model categories). Hence there is a lift in the diagram

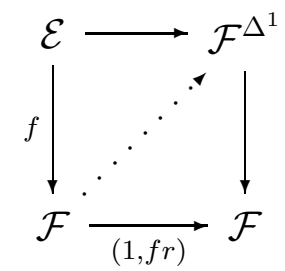

where the top map is the constant homotopy of $f$. This lift is a homotopy $1_{\mathcal{F}} \cong f r$. Thus $f$ is a homotopy equivalence as claimed. It follows immediately that for any object $\mathrm{U}, \mathcal{E}(U) \longrightarrow \mathcal{F}(U)$ is a homotopy equivalence of fibrant spectra and hence a weak equivalence.

In particular this shows that globally fibrant models are well-defined up to pointwise weak equivalence.

Elegant though this machinery may be, it is still in need of justification. The crucial test is that there should be a descent spectral sequence for the pointwise homotopy of a globally fibrant presheaf. The key step in setting up such a spectral sequence is an analysis of globally fibrant models for Eilenberg-Maclane spectra. So let A be a presheaf of abelian goups. An Eilenberg-Maclane presheaf of type $(A, n)$ is a presheaf of spectra $\mathcal{A}$ such that $\pi_{n} \mathcal{A}$ is isomorphic to $\mathrm{A}$ and $\pi_{k} \mathcal{A}$ is identically zero for $k \neq n$.

Proposition 3.18 Let $\mathcal{A}$ be an Eilenberg-Maclane presheaf of spectra of type $(A, n)$, with globally fibrant model $G \mathcal{A}$. Then for all $k \in \mathbb{Z}$ and all objects $U$ of the site,

$$
\pi_{k}(G \mathcal{A})(U) \cong H^{n-k}(U ; a A)
$$

We sketch a proof (see [22], Ch.4 for a proof that is more in the spirit of Thomason's "Scholium of Great Enlightenment"). It is convenient to work with spaces (=simplicial sets), so we first reduce to that case and use the closed model category structure for presheaves of spaces (see the end of this section). Since formal suspension and desuspension preserve globally fibrant 
models, we can assume $k \geq 0$. Since $\mathcal{A}$ can be assumed pointwise fibrant, and the zero-th space presheaf of $G \mathcal{A}$ is a globally fibrant presheaf of spaces, we reduce to the analogous statement for Eilenberg-Maclane presheaves of spaces. We will continue to use the notation $\mathcal{A}$ for such a presheaf.

Now let $\mathcal{P}_{s s}, \mathcal{P}_{\text {sag }}, \mathcal{P}_{c h}$ denote respectively the categories of presheaves of simplicial sets, simplicial abelian groups, and non-negatively graded chain complexes. There is an evident pair of adjoint functors

$$
\mathbb{Z}: \mathcal{P}_{\text {ss }} \longrightarrow \mathcal{P}_{\text {sag }}, i: \mathcal{P}_{\text {sag }} \longrightarrow \mathcal{P}_{\text {ss }}
$$

as well as a pair of mutually inverse equivalences of categories

$$
N: \mathcal{P}_{\text {sag }} \longrightarrow \mathcal{P}_{c h}, T: \mathcal{P}_{c h} \longrightarrow \mathcal{P}_{\text {sag }}
$$

(See [27]; $\mathrm{N}$ is the normalized chain complex functor.) Replacing $\mathcal{A}$ by the n-th Postnikov section of $\mathbb{Z} \mathcal{A}$, we can assume $\mathcal{A}$ is a simplicial abelian group. In fact up to natural pointwise weak equivalence we can even assume $\mathcal{A}=$ $T\left(C_{A}\right)$, where $C_{A}$ is the chain complex consisting of the presheaf $\mathrm{A}$ in degree $n$ and zero elsewhere. Now let $a A \longrightarrow I$. be an injective resolution of the sheaf $a A$, and form the chain complex $J_{A}$ which is zero above degree $n$, is equal to $I_{n-k}$ for $0<k<n$ and with $I_{0}=\operatorname{Ker}\left(I_{n} \rightarrow I_{n-1}\right)$. Then the natural map $C_{A} \longrightarrow J_{A}$ is a stalkwise homology isomorphism, and the pointwise homology of $J_{A}$ is given by $H_{k} J_{A}=H^{n-k}(U ; a A)$.

We claim that $i T J_{A}$ is a globally fibrant model for $\mathcal{A}$. Since homology in $\mathcal{P}_{\text {ch }}$ corresponds to homotopy in $\mathcal{P}_{\text {sag }}$, we have a stalkwise weak equivalence $\mathcal{A} \longrightarrow T\left(J_{A}\right)$. Finally, it is not hard to show that $i T J_{A}$ is globally fibrant for example, by using the argument for the analogous theorem in [15].

To construct the descent spectral sequence, we start from a Postnikov tower $\left\{P^{n} \mathcal{E}\right\}$ of the presheaf $\mathcal{E}$. Let $F_{n}$ denote the fibre of $P^{n} \mathcal{E} \longrightarrow P^{n-1} \mathcal{E}$. Easy formal arguments lead to a commutative ladder

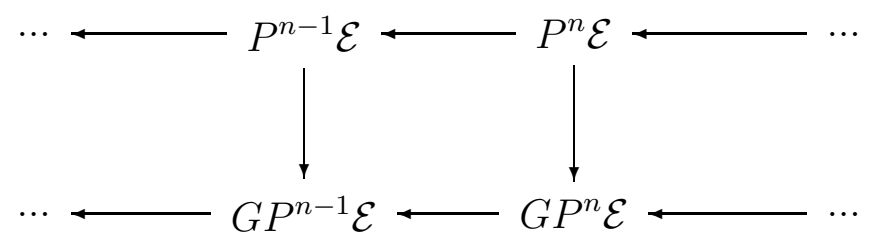

in which (i) the bottom row consist of global fibrations and globally fibrant objects; (ii) $\lim _{n} G P^{n} \mathcal{E}$ is globally fibrant; and (iii) the fibre of $G P^{n} \mathcal{E} \longrightarrow G P^{n-1} \mathcal{E}$ 
is a globally fibrant model $G F_{n}$ of the Eilenberg-Maclane presheaf $F_{n}$. Hence we have

$$
\pi_{k}\left(G F_{n}\right)(U)=H^{n-k}\left(U ; \tilde{\pi}_{n} \mathcal{E}\right)
$$

If we assume the cohomology groups on the right vanish for $n-k>>0$, then both towers are uniformly stable (see 3.1.3) and hence

$$
\mathcal{E}=\lim _{n} P^{n} \mathcal{E} \longrightarrow \lim _{n} G P^{n} \mathcal{E}
$$

is a stalkwise weak equivalence. In other words, $\lim _{n} G P^{n} \mathcal{E}$ is a globally fibrant model for $\mathcal{E}$. The tower of fibrations $G P^{n} \mathcal{E}$ then yields a spectral sequence with

$$
E_{1}^{p,-q}=H^{2 p-q}\left(X ; \tilde{\pi}_{p} \mathcal{E}\right)
$$

The indexing may look bizarre to some readers, but we can formally re-index the spectral sequence to get the usual form of the $E_{2}$-term (see [42],p.542; note the same peculiar indexing shows up if one constructs the Atiyah-Hirzebruch spectral sequence by a Postnikov resolution of the target instead of a skeletal filtration of the source). The result is:

Theorem 3.19 Let $\mathcal{E}$ be a presheaf of spectra on a site $\Phi$. Let $X$ be an object of the site and suppose $c d^{\Phi}(X ; \mathcal{E})=d<\infty$. Then there is a convergent right half-plane cohomology spectral sequence

$$
E_{2}^{p,-q}=H_{\Phi}^{p}\left(X ; \tilde{\pi}_{q} \mathcal{E}\right) \Rightarrow \pi_{q-p}(G \mathcal{E})(X)
$$

From this spectral sequence it is morally clear that the constructions of Jardine and Thomason must agree on a site with enough points, at least under finite cohomological dimension hypotheses. To prove this, we need to check two things.

Proposition 3.20 Let $\Phi$ be a site with enough points, $\mathcal{E}$ a presheaf of spectra on $\Phi$. Then the hypercohomology presheaf $\mathbb{H}^{\cdot}(-; \mathcal{E})$ is globally fibrant. If $c d^{\Phi} \mathcal{E}<\infty$, then the natural map $\mathcal{E} \longrightarrow \mathbb{H}^{\cdot}(-; \mathcal{E})$ is a stalkwise weak equivalence. 
Thus the hypercohomology presheaf of $\mathcal{E}$ is a globally fibrant model of $\mathcal{E}$. To get the stalkwise weak equivalence in the proposition, consider the map on stalks at $p$

$$
f: \operatorname{colim} \mathcal{E}(U) \longrightarrow \operatorname{colim} \mathbb{H} \cdot(U ; \mathcal{E})
$$

where the colimits are over the filtered category $\mathcal{N}_{p}^{o p}$. The finite cohomological dimension hypothesis ensures that the colimit of the descent spectral sequences for $\mathbb{H}^{\prime}(U ; \mathcal{E})$ converges to the homotopy of the colimit (Proposition 3.3). This colimit spectral sequence collapses to its vertical edge, and $\pi_{*} f$ can be identified with the natural isomorphism $\left(\pi_{*} \mathcal{E}\right)_{p} \cong\left(\tilde{\pi}_{*} \mathcal{E}\right)_{p}$.

The adjoint functor trick used in (3.14) shows that each cosimplicial term of the cosimplicial presheaf $T \cdot \mathcal{E}$ is a globally fibrant presheaf. One then has to show that the homotopy limit remains globally fibrant, using the techniques of $[6]$; see $[20], 3.3$.

We conclude this section with a discussion of the proof of Theorem 3.13. The theorem was proved first for presheaves of simplicial sets in [20], and then extended to spectra in [21], using the techniques of [5]. The key point is to get a closed model category structure on the category $\mathcal{P}_{\text {ssets }}$ of presheaves of simplicial sets.

In $\mathcal{P}_{\text {ssets }}$ the definition of stalkwise weak equivalence is not as simple as it is for spectra, because one cannot even define presheaves of homotopy groups without a compatible family of basepoints. Suppose that E is a presheaf of simplicial sets and $\mathrm{U}$ is an object of the site. Each choice of basepoint $e_{0} \in E(U)_{0}$ defines a compatible family of basepoints on the local site $\Phi / U$, because $\mathrm{U}$ is a terminal object of the latter. Hence for $n>0$ we get a presheaf of homotopy groups $\pi_{n}\left(\left.E\right|_{U} ; e_{0}\right)$ on $\Phi / U$. If $n=0$ we have a presheaf of sets $\pi_{0} E$ on $\Phi$. We then say that a map $f: E \longrightarrow F$ is a stalkwise weak equivalence if (i) $\tilde{\pi}_{0}(f)$ is an isomorphism of sheaves; and (ii) for all objects U, basepoints $e_{0} \in E(U)_{0}$, and $n>0$, the induced map $\tilde{\pi}_{n}\left(\left.E\right|_{U} ; e_{0}\right) \longrightarrow \tilde{\pi}_{n}\left(\left.F\right|_{U} ; f\left(e_{0}\right)\right)$ is an isomorphism of sheaves on $\Phi / U$.

It is not hard to check that if the site has enough points, then $f$ is a stalkwise weak equivalence if and only if $p^{*} E \longrightarrow p^{*} F$ is a weak equivalence for a sufficient set of points $p$. In this case several steps of the proof of Theorem 3.13 become much easier.

As in the case of spectra, the cofibrations are the pointwise cofibrations and the global fibrations are defined by the right lifting property with re- 
spect to acyclic cofibrations. Axioms CM1 and CM3 (as well as the mostly redundant requirement that the three classes are closed under composition and contain all identities) are then easily verified. If the site has enough points, CM2 is obvious (CM2 is also obvious for spectra). In the general case, however, one of the three implications of CM2 runs into a problem with basepoints, as the reader will quickly discover on inspection (see [20], 1.11 and 2.1). Half of CM4 is true by definition. It turns out that the other half follows formally from CM5 by a trick due to Joyal, using nothing more than the fact that cofibrations are stable under cobase change ([20], p. 64).

The crux of the matter, then, is CM5. This axiom is usually proved using Quillen's "small object argument". Consider first the problem of factoring a map $f: E \longrightarrow F$ as an acylic cofibration followed by a global fibration. To make the small object argument work, two ingredients are needed. First, we need to know that acyclic cofibrations are stable under (i) cobase change, and (ii) pseudo-filtered colimits. If the site has enough points, (i) is obvious ((i) is also obvious for spectra, because of the Mayer-Vietoris sequence in homotopy associated to a pushout square). The general case of (i) is much harder ([20], 2.2). Condition (ii) is clear in all cases.

Second, we need to know that there is a set $\mathrm{S}$ of acyclic cofibrations such that (i) S detects global fibrations (in the obvious sense); and (ii) S is "small", in a sense to be indicated. Here we fix an infinite cardinal number $\alpha$ greater than the cardinality of the set of morphisms of the underlying category of the site. Then for $\mathrm{S}$ we take a set of representatives of the isomorphism classes of acyclic cofibrations $A \longrightarrow B$ such that the cardinality of $B(U)_{n}$ is at most $\alpha$ for all objects $\mathrm{U}$ and all $n \geq 0$. The key fact is that this $\mathrm{S}$ detects global fibrations; see [20], Lemma 2.4, for the interesting proof.

Returning to an arbitrary morphism $f: E \longrightarrow F$, the "small object argument" (or "infinite glueing construction" in [11]) begins by forming the pushout

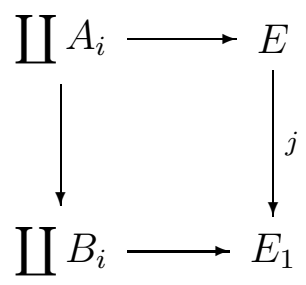


in which $A_{i} \longrightarrow B_{i}$ is ranging over all maps of elements of $\mathrm{S}$ into $f$. This yields a factorization $E \longrightarrow E_{1} \longrightarrow F$ in which the first map is an acyclic cofibration. The second map is not a global fibration, but at least all the lifts associated to the given $A_{i} \longrightarrow B_{i}$ have been "filled in". Iterating this construction some transfinite number of times (taking colimits at the limit ordinals) yields a factorization

$$
E \stackrel{i}{\longrightarrow} E_{\infty} \stackrel{p}{\longrightarrow} F
$$

in which $E_{\infty}$ is a certain colimit over some large ordinal. The point now is that if we choose this ordinal to be sufficiently large (bigger than the cardinality of the power set of the set of morphisms will do), then the cardinality bound defining the elements of $\mathrm{S}$ forces every map of an element $A \longrightarrow B$ of $\mathrm{S}$ into $f$ to factor through some lower stage of the colimit. Then the desired lift exists by construction.

The second part of CM5 can also be achieved by a small object argument, or by the following cheap trick. In the closed model category structure on simplicial sets, the factorizations of CM5 can be chosen in a functorial way. Hence any map of presheaves $f: E \longrightarrow F$ can be factored as

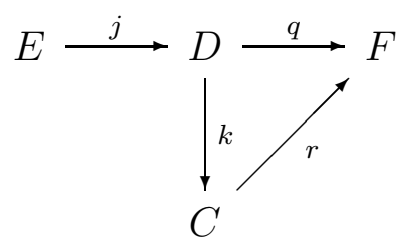

where $j$ is a cofibration, $q$ is a pointwise weak equivalence, and $q$ has been factored as an acylic cofibration $k$ followed by a global fibration $r$, using the part of CM5 already proved. Then $k j$ is a cofibration and $r$ is an acyclic global fibration (using CM2). See also the more interesting argument in [20], which shows that the acyclic fibration can be taken to be a pointwise weak equivalence.

\section{4 Čech hypercohomology spectra}

Our discussion of $\check{C}$ ech hypercohomology will be brief and somewhat imprecise. See [42],1, for details. Fix a site $\Phi$ and an object $\mathrm{X}$ of the site. Let 
$\mathcal{U}=\left\{U_{i} \longrightarrow X\right\}$ be a covering family. As a cosimplicial object, the complex defining ordinary $\check{C}$ ech cohomology (2.9) makes perfectly good sense for presheaves with values in any category with products. In particular, given a presheaf of spectra $\mathcal{E}$, we obtain in this way a cosimplicial spectrum $\check{C} \cdot(\mathcal{U} / X ; \mathcal{E})$. Taking an appropriate filtered colimit over a cofinal family of covers leads to the absolute $\check{C}$ ech complex $\check{C} \cdot(X ; \mathcal{E})$. The corresponding $\breve{C}$ ech hypercohomology spectra are then defined by

$$
\check{\mathbb{H}}(\mathcal{U} / X ; \mathcal{E})=\operatorname{holim}_{\Delta} \check{C} \cdot(\mathcal{U} / X ; \mathcal{E})
$$

and

$$
\check{\mathbb{H}}(X ; \mathcal{E})=\text { holim }_{\Delta} \operatorname{colim}_{\mathcal{U}} \check{C} \cdot(\mathcal{U} / X ; \mathcal{E})
$$

As with the Godement complex, each of these formulae lead to a descent spectral sequence with $E_{2}$-term given by the appropriate $\check{C}$ ech cohomology groups. Of course one cannot, in general, reverse the order of the holim and the colim in the second formula above. Using (3.3), however, we obtain:

Proposition 3.21 If $\mathcal{E}$ is pointwise bounded above, then the natural map

$$
\operatorname{colim}_{\mathcal{U}} \check{\mathbb{H}}^{\cdot}(\mathcal{U} / X ; \mathcal{E}) \longrightarrow \check{\mathbb{H}}^{\cdot}(X ; \mathcal{E})
$$

is a weak equivalence.

(Note that if $\pi_{q} \mathcal{E}=0$ for $q>m$, then for all $\mathcal{U}$ we have $\pi_{q} \check{\mathscr{H}}(\mathcal{U} / X ; \mathcal{E})=0$ for $q>m$.)

Example 3.22 Consider the étale site of a field $k$. We can take the finite Galois extensions of $k$ as cofinal system of covers. Then for $\mathcal{E}$ pointwise bounded above, we have (reverting to the notation of section 1)

$$
\operatorname{colim}_{\alpha} \mathbb{H}^{\cdot}\left(G_{\alpha} ; \mathcal{E}\right) \cong \mathbb{H}^{\cdot}(k ; \mathcal{E})
$$

where $G_{\alpha}$ ranges over the Galois groups of such extensions. More generally this works with any Galois extension $M$ of $k$ in place of the separable closure.

"Čech descent for sheaf cohomology" takes the following form: 
Theorem 3.23 Suppose that either $\mathcal{E}$ is stalkwise bounded above, or that there is a uniform bound on $c d(U ; \mathcal{E})$ (uniform in $U$, as $U$ ranges over the individual objects of all covering families in sight). Then there is a natural weak equivalence

$$
\mathbb{H}^{\prime}(X ; \mathcal{E}) \cong \check{\mathbb{H}}^{\prime}\left(\mathcal{U} / X ; \mathbb{H}^{\prime}(-; \mathcal{E})\right)
$$

Example 3.24 In the notation of the preceeding example, suppose that either $\mathcal{E}$ is stalkwise bounded above or that $\mathcal{E}$ is an $\ell$-torsion spectrum and $c d_{\ell} k=d<\infty$. Let $k^{\prime} / k$ be a Galois extension with group $G$. Then

$$
\mathbb{H}^{\prime}(k ; \mathcal{E}) \cong \mathbb{H}^{\prime}\left(G ; \mathbb{H}^{\prime}\left(k^{\prime} ; \mathcal{E}\right)\right)
$$

Note $c d_{\ell} k^{\prime} \leq d$

As a final and very pretty example we show how Artin's Theorem 2.9 extends to hypercohomology spectra ([42], Proposition 1.54).

Theorem 3.25 Suppose $X$ is quasi-projective over a noetherian ring of finite Krull dimension, and $\mathcal{E}$ is an additive presheaf of spectra on $X_{\text {ét }}$. Then there is a natural chain of weak equivalences between $\mathbb{H}^{\prime}{ }^{\prime} t(X ; \mathcal{E})$ and $\check{\mathbb{H}}^{\prime}{ }^{e} t(X ; \mathcal{E})$.

To prove this one first reduces to the case where $\mathcal{E}$ is pointwise bounded above, by using a Postnikov resolution. Then consider the natural maps

$$
\check{\mathbb{H}}^{\prime}{ }^{t}(X ; \mathcal{E}) \stackrel{\alpha}{\longrightarrow} \check{\mathbb{H}}^{\prime}{ }^{\prime}\left(X ; \mathbb{H}^{\prime}{ }_{e t}(-; \mathcal{E})\right) \stackrel{\beta}{\longleftarrow} \mathbb{H}^{\prime}{ }^{e} t(X ; \mathcal{E})
$$

Note that $\beta$ is a weak equivalence by Theorem 3.23. As for $\alpha$, note first that the $\check{C}$ ech hypercohomology spectral sequences converge, so it is enough to show that $\alpha$ is an isomorphism on $E_{2}$-terms - i.e., the map of coefficient presheaves $\pi_{q} \mathcal{E} \longrightarrow \pi_{q} \mathbb{H}^{\prime}{ }^{e} t(-; \mathcal{E})$ induces an isomorphism on $\breve{C}$ ech cohomology. But this map is an isomorphism on stalks by the second part of Proposition 3.20, and hence induces an isomorphism on sheaf cohomology of the associated sheaves. Then Artin's Theorem 2.9 completes the proof.

In conclusion, we note that it might be interesting to study Verdier hypercohomology spectra (see section 2.9). 


\section{Descent theorems in K-theory}

Let $\mathcal{E}$ be a presheaf of spectra on a site $\Phi, \mathrm{X}$ an object of the site. If the site has enough points, so that Thomason's hypercohomology spectrum $\mathbb{H}_{\Phi}{ }_{\Phi}(X ; \mathcal{E})$ is defined, then the problem of descent is to determine whether or not the natural map $\eta: \mathcal{E}(X) \longrightarrow \mathbb{H}^{\circ}(X ; \mathcal{E})$ is a weak equivalence. If it is a weak equivalence, then we get a descent spectral sequence as in section 3 , but now converging to $\pi_{*} \mathcal{E}(X)$. Typically $\mathrm{X}$ is a terminal object of the site, and in the cases of interest any proof of descent for $\mathrm{X}$ will prove descent for an arbitrary object of the site as well. So we may drop the assumption of enough points, and (bearing in mind Proposition 3.20) rephrase the descent problem in terms of Jardine's closed model category structure.

Descent Problem: Let $\eta: \mathcal{E} \longrightarrow G \mathcal{E}$ be a globally fibrant model for the presheaf $\mathcal{E}$. Is $\eta$ a pointwise weak equivalence?

Recall that the map $\eta$ is always a stalkwise weak equivalence, by definition.

Now let $\mathrm{X}$ be a separated noetherian regular scheme of finite Krull dimension,and let $\mathcal{E}=K$ denote the K-theory presheaf on one of the sites $X_{Z a r}, X_{N i s}, X_{e ́ t}$. Then $\mathrm{K}$ satisfies descent on $X_{Z a r}$ by the Brown-Gersten theorem, and satisfies descent on $X_{N i s}$ by a theorem of Nisnevich; these results are discussed in the first two sections below. Descent on the étale site is precisely the subject of the Lichtenbaum-Quillen conjectures. In this case, however, there are two complicating factors. The first is that, for technical reasons, we need to work at a fixed prime $\ell$ with the $\ell$-adic completions. The second and more serious complication is that it simply isn't true that Ktheory satisfies $\ell$-adic descent. In fact, in the form stated above descent fails even for finite fields $\mathrm{F}$, because the $\ell$-adic hypercohomology spectrum has a nonvanishing $\pi_{-1}$, isomorphic to $H_{\text {ét }}^{1}\left(\operatorname{Spec} F ; Z_{\ell}(0)\right) \cong Z_{\ell}$. A natural reaction to this annoyance is to replace $G K$ (or equivalently, $\mathbb{H}^{*}$ ) by its $(-1)$-connected cover. But then for example when $\mathrm{F}$ is a number field, we find that $\pi_{0}(G K)^{\wedge}$ contains a contribution from the Brauer group that is simply not present in $K_{0} F$. It seems that the best one can hope for is an equivalence above some dimension depending on the $\ell$-adic étale cohomological dimension $d$ of the scheme X.

$\ell$-adic Étale Descent Problem for K-theory: Is the globally fibrant model $\eta: K \longrightarrow G_{e t} K$ a pointwise weak equivalence after $\ell$-adic completion 
and passing to some connected cover?

One guess is that $\pi_{k} \eta^{\wedge}$ is an isomorphism for $k>2 d$; for another see [22], Conjectures 7.35 and 7.36. The Beilenson-Lichtenbaum conjectures suggest yet another guess; see for example [40].

Thomason's descent theorem [42], which forms the main subject of this paper, says that $\eta$ induces an isomorphism on "Bott-periodic" homotopy mod powers of $\ell$. Equivalently, $\hat{L}(\eta)$ is a weak equivalence, where $\hat{L}$ denotes Bousfield localization with respect to $\bmod \ell$ topological complex K-theory. Spectra of the form $\hat{L}(-)$ are always non-connective (or trivial!), so connectivity obstructions of the sort discussed above do not arise. The proof we will sketch has two main steps - (i) the proof for fields, all discussion of which is postponed to the final section 5; and (ii) the deduction of the general case from (i). With the Thomason-Jardine machinery of section 3 and the Nisnevich descent theorem in hand, the proof of step (ii) is remarkably simple.

For practical applications, the descent problem as formulated above is still missing two ingredients. First, the main point of establishing a pointwise weak equivalence $\mathcal{E} \longrightarrow G \mathcal{E}$ is not merely to get a descent spectral sequence, but to get one whose $E_{2}$-term is actually computable. This means that we need to at least identify the sheaves associated to the presheaves $\pi_{q} \mathcal{E}$. We state this as:

Descent Problem, Addendum: "Determine" the associated sheaves $\tilde{\pi}_{*} \mathcal{E}$.

In practice this amounts to identifying the stalks, and by the continuity theorem 2.11 this in turn reduces to to computing $\pi_{*} \mathcal{E}$ on the appropriate local rings. Thus in the K-theory setting, we need to know the K-theory of the Zariski, hensel, or strict hensel local rings, as the case may be. In the strict hensel case the answer is completely known, thanks to the Gillet-ThomasonSuslin-Gabber rigidity theorem and Suslin's theorem on algebraically closed fields. This is discussed further in section 4.11 below; in particular we show how Thomason's descent theorem yields a spectral sequence with the desired $E_{2}$-term.

Second, one needs to know that the descent spectral sequence converges. The cheapest way to ensure convergence is to assume that the presheaf (or even the site) in question has finite cohomological dimension. We generally do 
so in order to simplify the exposition. It is worth noting, however, that there are interesting cases where the cohomological dimension is infinite but one has convergence nevertheless. For example, suppose $\ell=2$ and $X=\operatorname{Spec} R$ with $\mathrm{R}$ either a number field admitting a real embedding or a ring of S-integers in such a field. Then $c d_{2}^{e ́ t} X=\infty$. Nevertheless, the 2-adic descent spectral sequence for $\mathbb{H}^{-} e ́ t(X ; K)$ converges - in fact, the filtration has length at most 3 and the spectral sequence collapses at $E_{4}$. This follows from the analogous statement for $\mathbb{R}$, together with classical calculations of Tate. As a result, many of the general results of the last section still apply (see Remark 3.6).

Finally, some comments may be in order concerning our scheme-theoretic hypotheses. The three K-theory descent theorems stated here all assume that $\mathrm{X}$ is separated and regular. The sole reason for this assumption is to ensure that $K X \cong K^{\prime} X$, where $K^{\prime} X$ is the $\mathrm{K}$-theory spectrum associated to the exact category of coherent sheaves on $X$. This is an instance of "reduction by resolution" [35], which in turn depends on Theorem 2.1. Thus the "separated and regular" hypothesis can be dropped if we are only interested in $K^{\prime} X$. It can also be dropped if we replace K-theory with Bass K-theory, using the deep theorem of Thomason [44].

For Thomason's étale descent theorem, we also assume that $\mathrm{X}$ is uniformly $\ell$-bounded (i.e. there is a uniform bound on $c d_{\ell} k$ as $k$ ranges over the residue fields of $\mathrm{X}$ ). One reason for this assumption (which is satisfied in most cases of interest) is to ensure that $c d_{\ell} X<\infty$, so that the descent spectral sequence converges. Moreover it is a property inherited by every object of the (restricted) étale site of X. Hence we are free to pass back and forth between hypercohomology presheaves $\grave{a}$ la Thomason and globally fibrant models $\grave{a}$ la Jardine, using Proposition 3.20.

Some further (but mild) restriction on the residue fields is necessary. Our assumption will be that the residue fields are " $\ell$-good" as defined in section 4.4. This hypothesis, which is taken from [22], could undoubtedly be weakened somewhat; it has the advantage that it is easily remembered and applied.

\subsection{Brown-Gersten descent}

Let $\mathrm{X}$ be a scheme, $\mathcal{E}$ a presheaf of spectra on the Zariski site of X. We say that $\mathcal{E}$ is a Mayer-Vietoris presheaf if for every pair of open sets $U, V \subset X$, the diagram 


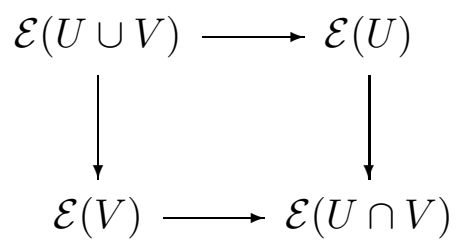

is a homotopy fibre square. Alternatively, we can express this as an excision condition for closed pairs. Given closed subschemes $\mathrm{Y}, \mathrm{Z}$ with $Z \subset Y$, let $\Gamma_{Y, Z} \mathcal{E}$ denote the presheaf assigning to each open $\mathrm{U}$ the homotopy fibre of $\mathcal{E}(U-Z) \longrightarrow \mathcal{E}(U-Y)$. We write $\Gamma_{Y}$ for $\Gamma_{Y, \phi}$. Then $\mathcal{E}$ satisfies excision if $\Gamma_{Y, Z} \mathcal{E}$ depends only on $Y-Z$ - that is, if $(Y, Z) \subset\left(Y^{\prime}, Z^{\prime}\right)$ then the natural map $\Gamma_{Y, Z} \mathcal{E} \longrightarrow \Gamma_{Y^{\prime}, Z^{\prime}} \mathcal{E}$ is a pointwise weak equivalence. It is easy to check that $\mathcal{E}$ is Mayer-Vietoris if and only if $\mathcal{E}$ satisfies excision.

Remark: Quillen's localization sequence for $K^{\prime}$-theory [35] shows that $K^{\prime}$ satisfies excision and hence defines a Mayer-Vietoris presheaf. If $\mathrm{X}$ is separated and regular, then the K-theory presheaf is also a Mayer-Vietoris presheaf, since then $K \cong K^{\prime}$.

The next theorem is due to Brown and Gersten [7].

Theorem 4.1 Let $X$ be a noetherian scheme of finite Krull dimension, and suppose that $\mathcal{E}$ is a Mayer-Vietoris presheaf of spectra on the Zariski site of $X$. Then $\mathcal{E}$ satisfies Zariski descent: any globally fibrant model

$$
\mathcal{E} \longrightarrow G_{Z a r} \mathcal{E}=\mathbb{H}^{\cdot} \text { Zar }(-; \mathcal{E})
$$

is a pointwise weak equivalence.

Corollary 4.2 If $X$ is a separated noetherian regular scheme the K-theory presheaf satisfies Zariski descent. In particular there is a convergent spectral sequence

$$
E_{2}^{p,-q}=H_{Z a r}^{p}\left(X ; \tilde{\pi}_{q} K\right) \Rightarrow \pi_{q-p} K(X)
$$

Here is a quick proof of the theorem, paraphrasing Thomason's exercise 2.5 [42]. Call a presheaf of spectra quasi-fibrant if it is pointwise weak equivalent to a globally fibrant presheaf. We will use the following easy facts: (a) if 
any two terms of a pointwise fibre sequence are quasi-fibrant, so is the third; and (b) a pseudo-filtered colimit of quasi-fibrant presheaves is quasi-fibrant (this follows from Proposition 3.7, since $X_{Z a r}$ is a noetherian site of finite cohomological dimension).

Let $\mathrm{Y}$ be a closed subscheme of $\mathrm{X}$. For each closed $Z \subset Y$ of codimension at least one, the sequence

$$
\Gamma_{Z} \mathcal{E} \longrightarrow \Gamma_{Y} \mathcal{E} \longrightarrow \Gamma_{Y, Z} \mathcal{E}
$$

is a pointwise fibre sequence by the "Quetzlcoatl lemma" (Thomason's picturesque term for the homotopy-theoretic analogue of the snake lemma). Passing to the colimit over the directed set of all such $\mathrm{Z}$ yields a pointwise fibre sequence

$$
\operatorname{colim}_{Z} \Gamma_{Z} \mathcal{E} \longrightarrow \Gamma_{Y} \mathcal{E} \longrightarrow C_{Y} \mathcal{E}
$$

where $C_{Y} \mathcal{E}=\operatorname{colim}_{Z} \Gamma_{Y, Z} \mathcal{E}$. If $\mathrm{Y}$ is irreducible with generic point $x$, we also write $\Gamma_{x} \mathcal{E}=C_{Y} \mathcal{E}$ (the reason for this apparently superfluous notation will appear when we consider Nisnevich descent). Using excision, Quetzlcoatl, and induction, one obtains:

Lemma 4.3 Suppose $Y$ has irreducible components $Y_{1}, \ldots, Y_{m}$. Then there is a pointwise weak equivalence

$$
\bigvee C_{Y_{i}} \mathcal{E} \stackrel{\cong}{\longrightarrow} C_{Y} \mathcal{E}
$$

Now let

$$
S^{p} \mathcal{E}=\operatorname{colim}_{\text {codim } Y \geq p} \Gamma_{Y} \mathcal{E}
$$

We will show by descending induction on $p$ that $S^{p} \mathcal{E}$ is quasi-fibrant. This will prove the theorem, since $S^{0} \mathcal{E}=\mathcal{E}$. To start the induction, note that if $\mathrm{X}$ has Krull dimension $n, S^{n+1} \mathcal{E}=*$. At the inductive step, define $C^{p} \mathcal{E}$ by the pointwise cofibre sequence

$$
S^{p+1} \mathcal{E} \longrightarrow S^{p} \mathcal{E} \longrightarrow C^{p} \mathcal{E}
$$

It is enough to show that $C^{p} \mathcal{E}$ is quasi-fibrant. 
Lemma 4.4 There is a pointwise weak equivalence

$$
\bigvee_{\operatorname{codim} x=p} \Gamma_{x} \mathcal{E} \longrightarrow C^{p} \mathcal{E}
$$

The proof is easy from Lemma 4.3. Thus we have reduced to showing that $\Gamma_{x} \mathcal{E}$ is quasi-fibrant for all $x \in X$.

Lemma 4.5 Let $i: x \rightarrow X$ denote the inclusion. Then the natural map $\Gamma_{x} \mathcal{E} \longrightarrow i_{\#} i^{\#} \Gamma_{x} \mathcal{E}$ is a pointwise weak equivalence.

To prove this lemma, it is enough to check (i) $\left(\operatorname{colim}_{Z} \Gamma_{Y, Z} \mathcal{E}\right)(U)$ is weakly contractible if $x$ is not in $\mathrm{U}$; and (ii) $\left(\operatorname{colim}_{Z} \Gamma_{Y, Z} \mathcal{E}\right)(U) \longrightarrow\left(\operatorname{colim}_{Z} \Gamma_{Y, Z} \mathcal{E}\right)(W)$ is a weak equivalence whenever $x \in W \subset U$. Condition (i) is obvious. For condition (ii), consider the diagram

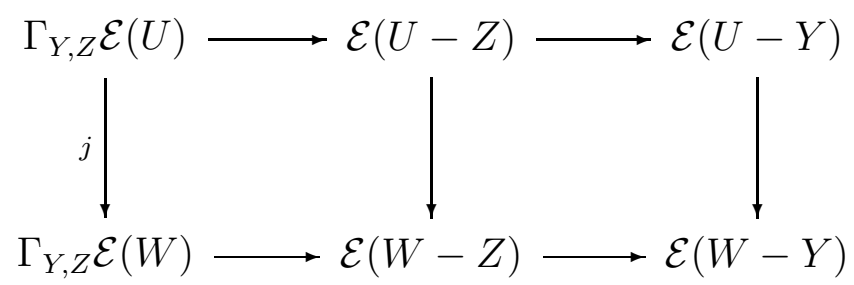

Note that $(W-Z) \cap(U-Y)=W-Y$. If we have $Y-Z \subset W$, then $U-Z=(U-Y) \cup(W-Z)$ and hence $j$ is a weak equivalence by a direct application of the Mayer-Vietoris condition. But the set of $\mathrm{Z}$ satisfying $Y-Z \subset W$ is easily seen to be cofinal, so $j$ is a weak equivalence after passage to the colimit, proving (ii).

A presheaf on the Zariski site of a field is quasi-fibrant if and only if its value on the empty set is weakly contractible. It follows that $i^{\#} \Gamma_{x} \mathcal{E}$ is quasifibrant, and since direct image functors preserve quasi-fibrant presheaves, this completes the proof of Theorem 4.1.

\subsection{Nisnevich descent}

Let X be a noetherian scheme of finite Krull dimension. By excision data we mean a morphism $f: U \longrightarrow V$ between objects of the Nisnevich site (recall that the underlying category is the same as that of the étale site), together 
with a closed subscheme $Z \subset V$ such that $U \times_{V} Z \longrightarrow Z$ is an isomorphism. A presheaf of spectra $\mathcal{E}$ on $X_{N i s}$ satisfies Nisnevich excision if for all such excision data, the square

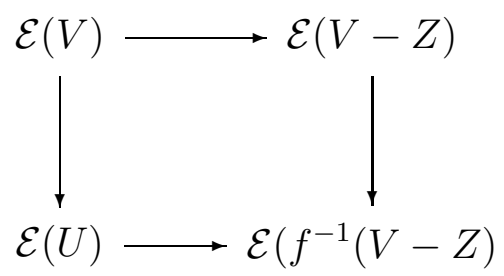

is a homotopy fibre square, and $\mathcal{E}(\phi) \cong *$. It is easy to check that then $\mathcal{E}$ is additive - that is, $\mathcal{E}(U \amalg V) \cong \mathcal{E}(U) \times \mathcal{E}(V)$ - and that the restriction of $\mathcal{E}$ to the Zariski site of any object $U$ satisfies Zariski excision (i.e. the MayerVietoris-condition of the preceeding section). It is also clear that $K^{\prime}$-theory satisfies Nisnevich excision, and hence so does K-theory if $\mathrm{X}$ is separated and regular.

The following theorem is the Nisnevich descent theorem [34].

Theorem 4.6 Let $X$ be a noetherian scheme of finite Krull dimension, $\mathcal{E}$ a presheaf of spectra on the Nisnevich site of $X$. If $\mathcal{E}$ satisfies Nisnevich excision, then $\mathcal{E}$ satisfies Nisnevich descent - that is, the globally fibrant model

$$
\mathcal{E} \longrightarrow G_{N i s} \mathcal{E}=\mathbb{H}^{\cdot}{ }_{N i s}(-; \mathcal{E})
$$

is a pointwise weak equivalence.

Corollary 4.7 If $X$ is a separated noetherian regular scheme of finite Krull dimension, the K-theory presheaf satisfies Nisnevich descent.

The proof that we will sketch here follows [22], and very closely parallels the proof of the Brown-Gersten theorem. For each object $U \longrightarrow X$ of $X_{N i s}$ and each closed subscheme $Y \subset U$, we can form the spectra $\Gamma_{Y} \mathcal{E}(U)$ and $C_{Y} \mathcal{E}(U)$ as before. Note that these are not presheaves on $X_{N i s}$. We can, however, define for each $x \in X$ a presheaf $\Gamma_{x} \mathcal{E}$ by

$$
\Gamma_{x} \mathcal{E}(\pi: U \longrightarrow X)=C_{\overline{\pi^{-1} x}} \mathcal{E}(U)
$$


The spectrum $S^{p} \mathcal{E}(U)$ we defined in the Zariski case is functorial in U, so we also get a presheaf $S^{p} \mathcal{E}$ on $X_{N i s}$. Let us again define $C^{p} \mathcal{E}$ by the pointwise cofibre sequence

$$
S^{p+1} \mathcal{E} \longrightarrow S^{p} \mathcal{E} \longrightarrow C^{p} \mathcal{E}
$$

Lemma 4.8 There is a pointwise weak equivalence

$$
\bigvee_{\operatorname{codim} x=p} \Gamma_{x} \mathcal{E} \longrightarrow C^{p} \mathcal{E}
$$

The proof is easy from Lemma 4.3. Thus we have again reduced to showing that $\Gamma_{x} \mathcal{E}$ is quasi-fibrant.

Lemma 4.9 Let $i: x \rightarrow X$ denote the inclusion. Then the natural map $\Gamma_{x} \mathcal{E} \longrightarrow i_{\#} i^{\#} \Gamma_{x} \mathcal{E}$ is a pointwise weak equivalence.

Assuming this for the moment, the proof of Theorem 4.6 is completed as follows. A presheaf of spectra on the Nisnevich site of a field is quasi-fibrant if and only if it is additive (the proof is straightforward - this is the only place where the Nisnevich topology is used!). One easily checks that $\Gamma_{x} \mathcal{E}$ is additive and hence also $i^{\#} \Gamma_{x} \mathcal{E}$ is additive. Since direct images preserve quasi-fibrant presheaves, this completes the proof.

We briefly indicate the proof of Lemma 4.9, so the reader can at least see where the Nisnevich excision condition comes in. By unravelling the definitions of $i_{\#}$ and $i^{\#}$, one reduces to checking the following:

Lemma 4.10 Suppose $f: U \longrightarrow V$ is a morphism in $X_{N i s}$ and $z \in V$. Suppose that $f^{-1} z$ consists of a single point $y$, and that $y \rightarrow z$ is an isomorphism of schemes (i.e., an isomorphism on residue fields). Then the induced map $C_{\bar{z}} \mathcal{E}(V) \longrightarrow C_{\bar{y}} \mathcal{E}(U)$ is a weak equivalence.

If $\bar{y} \longrightarrow \bar{z}$ is an isomorphism of schemes, this follows by a direct application of Nisnevich excision. But we can always reduce to this case because $\bar{y} \longrightarrow \bar{z}$ is an isomorphism on generic points by assumption, and hence restricts to an isomorphism between suitable open subschemes of $\bar{y}, \bar{z}$. See [22],Lemma 7.21 , for further details. 


\subsection{Gabber rigidity and the K-theory sheaf}

On the étale site, the $\ell$-adic K-theory sheaf is explicitly known. Let

$$
R_{n}=\mathbb{Z}[t] / t^{\ell^{n}}-1
$$

and let $\mu_{\ell^{n}}$ denote the sheaf on the big étale site (all schemes) represented by Spec $R_{n}$. In other words, $\mu_{\ell^{n}}(U)$ is the group of units of order dividing $\ell^{n}$ in the ring of regular functions on $U$. Next, let $\beta_{n} \in \pi_{2}\left(B \mathbb{Z} / \ell^{n} ; \mathbb{Z} / \ell^{n}\right)$ correspond to the standard generator under the Bockstein isomorphism

$$
\pi_{2}\left(B \mathbb{Z} / \ell^{n} ; \mathbb{Z} / \ell^{n}\right) \cong \pi_{1} B \mathbb{Z} / \ell^{n}=\mathbb{Z} / \ell^{n}
$$

The natural map

$$
\Sigma^{\infty} \mathrm{BZ} / \ell_{+}^{n} \longrightarrow K R_{n}
$$

then yields a "Bott element" in $\pi_{2}\left(K R_{n} ; \mathbb{Z} / \ell^{n}\right)$, also denoted $\beta_{n}$.

Now define a map of presheaves $e: \mu \ell^{n} \longrightarrow \pi_{2}\left(K ; \mathbb{Z} / \ell^{n}\right)$ by

$$
e\left(f: U \longrightarrow \operatorname{Spec} R_{n}\right)=f^{*} \beta_{n}
$$

If $\mathrm{U}$ is affine - say $U=\operatorname{Spec} S$ - then $e(f)$ is just the image of $\beta_{n}$ under the composite

$$
B \mathbb{Z} / \ell^{n} \longrightarrow B G L_{1} R_{n} \longrightarrow B G L_{1} S \longrightarrow B G L S^{+}
$$

From this description it is clear that $e$ is a group homomorphism. Using the ring spectrum structure on $K\left(-; \mathbb{Z} / \ell^{n}\right)$, we obtain more generally maps of presheaves

$$
e_{i}: \mathbb{Z} / \ell^{n}(i) \equiv \otimes^{i} \mu \ell^{n} \longrightarrow \pi_{2 i} K\left(-; \mathbb{Z} / \ell^{n}\right)
$$

Proposition 4.11 Let $X$ be a scheme over $\mathbb{Z}[1 / \ell]$. Then the maps $e_{i}$ induce isomorphisms of sheaves

$$
\mathbb{Z} / \ell^{n}\left(\frac{q}{2}\right) \stackrel{\cong}{\longrightarrow} \tilde{\pi}_{q} K\left(-; \mathbb{Z} / \ell^{n}\right)
$$

The group on the left is to be interpreted as zero if $q$ is odd. The proof of the proposition depends on the Gabber Rigidity Theorem ([13]; earlier versions are due to Gillet-Thomason [14] and Suslin ([39])): 
Theorem 4.12 Let $R$ be a hensel local ring with residue field $k$ of characteristic different from $\ell$. Then the reduction map $K R \longrightarrow K k$ is a weak equivalence on $\ell$-adic completions.

Combining Gabber rigidity and Suslin's theorem 1.1 shows that for any strictly henselian ring $S$ over $\mathbb{Z}[1 / \ell], \mathbb{Z} / \ell^{n}(q / 2) \cong \pi_{q}\left(K S ; \mathbb{Z} / \ell^{n}\right)$. Hence by the continuity theorem 2.11, $e_{i}$ induces an isomorphism on stalks. This proves the proposition.

In other words, we have settled the question raised in the "Addendum" to the descent problem mentioned in the introduction. As a consequence the $\ell$-adic descent spectral sequence for hypercohomology with coefficients in K-theory takes the form

$$
E_{2}^{p,-q}=H_{e t}^{p}\left(X ; Z_{\ell}\left(\frac{q}{2}\right)\right) \Rightarrow \pi_{q-p}\left(\mathbb{H}^{\dot{e}}{ }_{e t}(X ; K)^{\wedge}\right)
$$

where as always étale cohomology means continuous cohomology in the sense of Jannsen [19] and the groups on the right are the homotopy groups of the completion, not the completion of the homotopy groups. To see why we get continous cohomology, note first that

$$
\left(\mathbb{H}^{\prime}{ }^{\prime} e_{t}(X ; K)\right)^{\wedge}=\text { holim }_{n} \mathbb{H}^{\prime}{ }^{\prime}(X ; K) \wedge M \mathbb{Z} / \ell^{n}=\operatorname{holim}_{n} \mathbb{H}^{\prime}{ }^{e}\left(X ; K \wedge M \mathbb{Z} / \ell^{n}\right)
$$

by (3.11), and this in turn is equivalent to

$$
\operatorname{holim}_{n} \operatorname{holim}_{\Delta} T^{\cdot}\left(K\left(-; \mathbb{Z} / \ell^{n}\right)\right)(X)=\text { holim }_{\Delta} \operatorname{holim}_{n} T^{\cdot}\left(K\left(-; \mathbb{Z} / \ell^{n}\right)\right)(X)
$$

Therefore we get a spectral sequence whose $E_{2}$-term is the cohomotopy of the cosimplicial abelian group $\pi_{*}$ holim $_{n} T^{\cdot}\left(K\left(-; \mathbb{Z} / \ell^{n}\right)\right)(X)$. Now

$$
\pi_{q} T \cdot\left(K\left(-; \mathbb{Z} / \ell^{n}\right)\right)=T \cdot \pi_{q}\left(K\left(-; \mathbb{Z} / \ell^{n}\right)\right)=T \cdot \mu^{n}\left(\frac{q}{2}\right)
$$

Since the reduction maps $\mu_{\ell^{n+1}} \rightarrow \mu_{\ell^{n}}$ are epimorphisms of sheaves, and the composite functor $\Gamma(X,-) \circ T^{*}$ is exact, it follows that the Milnor $\lim ^{1}$ vanishes and

$$
\pi_{*} \operatorname{holim}_{n} T \cdot\left(K\left(-; \mathbb{Z} / \ell^{n}\right)\right)(X)=\lim _{n} \pi_{q} T \cdot\left(K\left(-; \mathbb{Z} / \ell^{n}\right)\right)
$$

Since $T$ takes values in acyclic sheaves, it also follows that $T \cdot \pi_{q}\left(K\left(-; \mathbb{Z} / \ell^{n}\right)\right)$

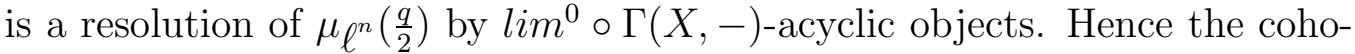
motopy yields continous cohomology as claimed. 


\subsection{Thomason's étale descent theorem}

We recall the following definition from Section 1: a field $F$ is $\ell$-good if char $F \neq \ell$ and $F$ has finite transcendence degree over a subfield E satisfying $c d_{\ell} E_{\infty} \leq 1$, where $E_{\infty}$ is obtained from $E$ by adjoining all the $\ell$-power roots of unity; and with $\sqrt{-1} \in E$ if $\ell=2$. A scheme $X$ is then said to be $\ell$-good if all of its residue fields are $\ell$-good.

Recall also (see Theorem 2.8) that a scheme $\mathrm{X}$ is uniformly $\ell$-bounded if there is a uniform bound $d<\infty$ on the étale cohomological dimension of the residue fields of $\mathrm{X}$. For example, if $\mathrm{X}$ is "uniformly $\ell$-good" in the sense that the transcendence degrees occurring in the definition of $\ell$-good scheme are uniformly bounded (e.g. any variety over an $\ell$-good field), then $\mathrm{X}$ is uniformly $\ell$ - bounded.

We can now state the descent theorem of Thomason [42]:

Theorem 4.13 Let $X$ be a separated noetherian regular scheme of finite Krull dimension. Assume that $X$ is $\ell$-good and uniformly $\ell$-bounded. Then the natural map $K X \longrightarrow \mathbb{H}^{\cdot}{ }^{\prime}(X ; K)$ induces a weak equivalence

$$
\hat{L} K(X) \stackrel{\cong}{\longrightarrow} \hat{L} \mathbb{H}^{\cdot} e^{\prime}(X ; K)
$$

Equivalently, in the terminology of Jardine, the localization of globally fibrant models

$$
\hat{L} K \longrightarrow \hat{L}\left(G_{e ́ t} K\right)
$$

is a pointwise weak equivalence on $X_{\text {ét }}$.

Corollary 4.14 There is a convergent descent spectral sequence

$$
E_{2}^{p,-q}=H_{e ́ t}^{p}\left(X ; Z_{\ell}\left(\frac{q}{2}\right)\right) \Rightarrow \pi_{q-p} \hat{L} K X
$$

For the corollary, note that $\hat{L}_{\mathbb{H}}{ }^{\circ}$ ét $(X ; K) \cong\left(\mathbb{H}^{\cdot}(X ; L K)\right)^{\wedge}$ by Corollary 3.12. The indicated form of the $E_{2}$-term then follows from Gabber rigidity as in section (4.11).

The two forms of Theorem 4.13 are equivalent by Proposition 3.20, and because all of the hypotheses on $\mathrm{X}$ are inherited by any object $U \rightarrow X$ of the (restricted) étale site of $\mathrm{X}$. 
Now, assuming Theorem 4.13 has been proved for $\ell$-good fields, the general case is a rather easy consequence of the machinery thus far assembled. It will be convenient to use the abbreviation

$$
\mathcal{L}=L K \wedge M \mathbb{Z} \ell=K \wedge L M \mathbb{Z} / \ell=K \wedge L S^{0} \wedge M \mathbb{Z} / \ell
$$

Then it is enough to show that the globally fibrant model $\eta: \mathcal{L} \longrightarrow G_{\text {ét }} \mathcal{L}$, which is by definition a stalkwise weak equivalence for the étale topology, is a pointwise weak equivalence (that is, a stalkwise weak equivalence for the chaotic topology!). Let us first show:

Lemma $4.15 \eta: \mathcal{L} \longrightarrow G_{\text {ét }} \mathcal{L}$ is a stalkwise weak equivalence for the Nisnevich topology.

Proof: By continuity, this reduces to showing that when $X=\operatorname{Spec} R, R$ a henselian ring, the map $\mathcal{L} X \longrightarrow\left(G_{\text {ét }} \mathcal{L}\right)(X)=\mathbb{H}^{\circ}$ ét $(X ; \mathcal{L})$ is a weak equivalence. Let $k$ as usual denote the residue field of the maximal ideal and let $x=$ Spec $k$. Consider the commutative diagram

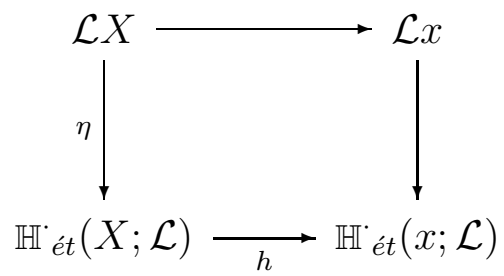

The top map is a weak equivalence by Gabber rigidity, and the righthand map is a weak equivalence by our assumption that the theorem has been proved for fields. The bottom map is also a weak equivalence. To see this, note that $\mathcal{L}$ is a presheaf on the big étale site, and $h$ factors as

$$
\mathbb{H}^{\cdot}{ }^{e} t\left(X ; \mathcal{L}_{X}\right) \stackrel{a}{\longrightarrow} \mathbb{H}^{\cdot} e^{\prime}\left(x ; j^{\#}\left(\mathcal{L}_{X}\right)\right) \stackrel{b}{\longrightarrow} \mathbb{H}^{\circ} e ́ t\left(x ; \mathcal{L}_{x}\right)
$$

Here the notations $\mathcal{L}_{X}, \mathcal{L}_{x}$ indicate restriction from the big étale site to the appropriate small site, and $j: x \rightarrow X$ is the inclusion. The first map is a weak equivalence by a spectral sequence comparison argument using Theorem 2.5. The second map is induced by the map of presheaves $j^{\#}\left(\mathcal{L}_{X}\right) \longrightarrow \mathcal{L}_{x}$ (which exists because $\mathcal{L}$ is a presheaf on the big site). This map is a stalkwise weak equivalence by Gabber rigidity, so $b$ is a weak equivalence as claimed. Hence $\eta$ is a weak equivalence, completing the proof of the lemma. 
Now consider the commutative diagram of presheaves

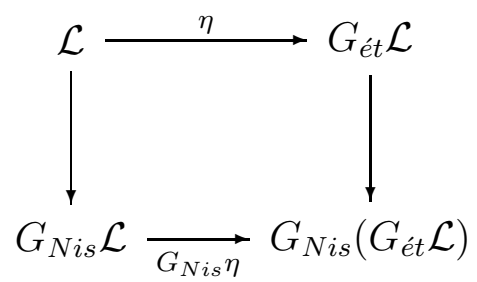

We wish to show that $\eta$ is a pointwise weak equivalence. The lefthand map is a pointwise weak equivalence by the Nisnevich descent theorem. The righthand map is a pointwise weak equivalence since $G_{e ́ t} \mathcal{L}$ is globally fibrant on the Nisnevich site (because the étale topology is finer than the Nisnevich topology; see Proposition 3.14). Since $\eta$ is a Nisnevich stalkwise weak equivalence by the lemma, the induced map $G_{N i s} \eta$ on globally fibrant models is a pointwise weak equivalence (Proposition 3.17). This completes the proof of Theorem 4.13 - assuming that it has been verified for fields, as will be done in the next section.

In conclusion, we remark that Thomason's original proof used the BrownGersten theorem, as Nisnevich descent was not available at the time. This approach does not work quite so neatly, because of the lack of an analogue of Gabber rigidity. See [42] or [22] for the Zariski descent proof. See also [44].

\section{The proof of Thomason's theorem for fields}

In this final section we sketch the proof of Theorem 4.13 for fields, following Chapter 7 of [22]. Since we no longer need to consider general schemes, we write in terms of rings rather than schemes and use the following notation: $\mathrm{F}$ is a field, and $\mathrm{L}$ is a finite separable extension of $\mathrm{F}$ - usually Galois with group G. X is a spectrum, a G-spectrum or a presheaf of spectra on the étale site of $\mathrm{F}$. Let $k(-)$ denote the $\bmod \ell \mathrm{K}$-theory presheaf $K \wedge M \mathbb{Z} / \ell$. In order to avoid distracting technicalities, we assume $\ell>3$.

Thus our goal is to show that the natural map $k F \longrightarrow \mathbb{H}^{\cdot}(F ; k)$ induces a weak equivalence $L k F \longrightarrow L \mathbb{H}^{\prime}(F ; k)$, or equivalently

$$
A^{-1} k F \stackrel{\cong}{\longrightarrow} A^{-1} \mathbb{H}^{\cdot}(F ; k) \cong \mathbb{H}^{\cdot}\left(F ; A^{-1} k\right)
$$


Here $\mathrm{A}$ is the Adams self-map of the Moore spectrum, and since $c d_{\ell} F<\infty$ we are free to commute $A^{-1}$ and $\mathbb{H}^{\cdot}$ by Theorem 3.7. A theorem of Snaith and Dwyer says that inverting the Adams map is equivalent to inverting the Bott element $\beta_{\ell_{-1}} \in k_{2 \ell_{-2}} F$. By a standard (and trivial) transfer argument we can assume $\mathrm{F}$ contains the $\ell$-th roots of unity. Thus we have a Bott element $\beta \in \pi_{2} k F$, and Theorem 4.13 for fields is equivalent to:

Theorem 5.1 Let $F$ be an $\ell$-good field containing the $\ell$-th roots of unity. Then $k F \longrightarrow \mathbb{H}^{\circ}(F ; k)$ induces a weak equivalence on Bott-periodic spectra

$$
\beta^{-1} k F \longrightarrow \beta^{-1} \mathbb{H}^{\cdot}(F ; k) \cong \mathbb{H}^{\cdot}\left(F ; \beta^{-1} k\right)
$$

The mapping telescopes in this theorem are to be interpreted in terms of the evident $k F$-module spectrum structures. In particular $\mathbb{H}^{\cdot}(F ; k)$ is a $k F$-module spectrum with multiplication

$$
k F \wedge \mathbb{H}^{\cdot}(F ; k) \longrightarrow \mathbb{H}^{\cdot}(F ; k F \wedge k) \longrightarrow \mathbb{H}^{\cdot}(F ; k)
$$

Before we can even outline the proof, we need some preliminaries on the hypernorm and hypertransfer (subsections 1-4). The fifth subsection outlines the proof modulo two key lemmas, whose proofs are sketched in the sixth and last subsection.

\subsection{Hyperhomology spectra}

Suppose $\mathrm{G}$ is a discrete group and $\mathrm{X}$ is a G-spectrum. The hyperhomology spectrum $\mathbb{H} .(G ; X)$ is the homotopy colimit of the G-action, which we can take to mean the spectrum $E G_{+} \wedge_{G} X$. Filtering EG by skeleta, one easily obtains a first quadrant homology spectral sequence

$$
E_{p, q}^{2}=H_{p}\left(G ; \pi_{q} X\right) \Rightarrow \pi_{p+q} \mathbb{H} .(G ; X)
$$

Let $\mathcal{F}(X, Y)$ denote the function spectrum for spectra $\mathrm{X}, \mathrm{Y}$. Then if $\mathrm{Y}$ has trivial G-action, there is a more or less obvious universal coefficient formula

$$
\mathbb{H}^{\cdot}(G ; \mathcal{F}(X, Y)) \cong \mathcal{F}(\mathbb{H} .(G ; X), Y)
$$




\section{$5.2 \quad$ The hypernorm}

Let $\mathrm{G}$ be a finite group, $\mathrm{X}$ a G-spectrum. There is a "norm" map $N: X \rightarrow X$, in the stable category that is just the sum of the actions of the various elements of G. An explicit, functorial strict representative of this map is given by the composite

$$
X \stackrel{\Delta_{G}}{\longrightarrow} \operatorname{Map}\left(G_{+}, X\right) \stackrel{\phi}{\longleftarrow} G_{+} \wedge X \stackrel{\nabla}{\longrightarrow} X
$$

where $\Delta_{G}(x)(g)=g x, \nabla$ is the ordinary fold map, and $\phi(g \wedge x)(h)=x$ if $h=g$ and $\phi(g \wedge x)(h)=*$ otherwise. Note that $\operatorname{Map}\left(G_{+}, X\right)$ and $G_{+} \wedge X$ are just the product and wedge respectively of $|G|$ copies of X. Hence $\phi$ is a weak equivalence and the diagram represents a map in the stable category.

Now observe that there are $G \times G$-actions on all four terms of the above diagram, such that the three maps are $G \times G$-equivariant - although the actions on the two instances of X are not the same! Explicitly, for $(g, h) \in$ $G \times G$ we have

$\operatorname{Map}(G, X): \quad[(g, h) \psi](a)=h \psi\left(h^{-1} a g\right)$

$G_{+} \wedge X:(g, h)(a \wedge x)=h a g^{-1} \wedge h x$

$\mathrm{X}$ (in source): $(g, h) x=g x$

$\mathrm{X}$ (in target): $(g, h) x=h x$

Thus the norm $\mathrm{N}$ is a $G \times G$-equivariant map.

There is a functor from $G \times G$-spectra to spectra that takes the homotopy limit with respect to the second copy of $\mathrm{G}$ and then the homotopy colimit with respect to the first copy of G. Equivalently, we can think of this functor as

$$
E G_{+}^{1} \wedge_{G^{1}}\left(\operatorname{Map}_{G^{2}}\left(E G_{+}^{2}, Y\right)\right)
$$

for a $G \times G$-spectrum $Y$. Here the superscripts serve to indicate which copy of $\mathrm{G}$ is acting. Applying this to the norm map and making use of the augmentation $B G_{+} \longrightarrow S^{0}$ yields

$E G_{+} \wedge_{G} X \longrightarrow E G_{+} \wedge_{G}\left(M a p\left(B G_{+}, X\right) \longrightarrow B G_{+} \wedge \operatorname{Map}^{G}\left(E G_{+}, X\right) \longrightarrow \operatorname{Map}^{G}\left(E G_{+}, X\right)\right.$

This composite is the hypernorm which we write as 


$$
N_{h}: \mathbb{H} .(G ; X) \longrightarrow \mathbb{H}^{\cdot}(G ; X)
$$

It has the property that the composite map

$$
X \longrightarrow \mathbb{H} .(G ; X) \longrightarrow \mathbb{H}^{\cdot}(G ; X) \longrightarrow X
$$

is the ordinary norm $\mathrm{N}$. As a consequence we have:

Lemma 5.2 Suppose the Tate cohomology groups $\hat{H}^{p}\left(G ; \pi_{q} X\right)$ vanish for all $p, q$. Then the hypernorm is a weak equivalence:

$$
N_{h}: \mathbb{H} .(G ; X) \stackrel{\cong}{\longrightarrow} \mathbb{H}^{\cdot}(G ; X)
$$

For both spectral sequences collapse to an edge, and the induced map on homotopy can be identified with the algebraic norm map

$$
n:\left(\pi_{q} X\right)_{G} \longrightarrow\left(\pi_{q} X\right)^{G}
$$

But $\operatorname{Ker} n=\hat{H}^{-1}$ and Coker $n=\hat{H}^{0}$, whence the lemma.

\subsection{An analogue for spectra of a theorem of Tate}

Let $\mathrm{G}$ be a finite group. Recall that Tate cohomology is defined in the following way. Given a G-module $\mathrm{M}$, let $P$. and $I$ denote the standard projective and injective resolutions (respectively) of $\mathrm{M}$. The Tate complex $\tau^{\cdot} M$ is obtained by splicing together $(P .)_{G}$ and $\left(I^{\cdot}\right)^{G}$ via the norm map

$$
\sigma:\left(P_{0}\right)_{G} \longrightarrow M_{G} \stackrel{n}{\longrightarrow} M^{G} \longrightarrow\left(I^{0}\right)^{G}
$$

In other words, $\tau^{*} M$ is the mapping cone of $\sigma$. Tate cohomology $\hat{H}^{*}(G ; M)$ is then defined as the cohomology of this complex.

The same construction works for hypercohomology of cochain complexes - given a cochain complex $C$. (which we picture running up the $q$-axis, in the upper half of the $(p, q)$-plane) we get a second quadrant double complex $\left(P^{* \cdot}\right)_{G}\left(C^{\cdot}\right)$ and a first quadrant double complex $\left(I_{. .}\right)^{G}\left(C^{\cdot}\right)$ which can be spliced to produce an upper half-plane double complex $\tau^{*}\left(G ; C^{\cdot}\right)$ of cohomological type.

Now suppose $\mathrm{G}$ is a profinite group and $\mathrm{M}$ is a discrete $\mathrm{G}$-module. We say that $c d M \leq d$ if for all open subgroups $\mathrm{V}$ of $\mathrm{G}, H^{n}(V ; M)=0$ for $n>d$. Then there is the following lemma of Tate ([37], Annexe 1 to Chapitre I): 
Lemma 5.3 The discrete $G$-module $M$ satisfies $c d M=0$ if and only if for all open normal subgroups $U$ of $G, \hat{H}^{*}\left(G / U ; M^{U}\right) \equiv 0$.

Now suppose $c d M \leq d$. Then one easily constructs a finite resolution

$$
0 \longrightarrow M \longrightarrow N^{0} \longrightarrow N^{1} \longrightarrow \ldots \longrightarrow N^{d} \longrightarrow 0
$$

with $c d N^{i}=0$ for all $i$ (Tate, loc. cit.). Fix an open normal subgroup $\mathrm{U}$ and form the associated Tate double complex $T^{* \cdot}\left(G / U ;\left(N^{*}\right)^{U}\right)$ as above. Since the horizontal cohomology of this complex vanishes by Tate's lemma, the cohomology of the total complex is also trivial. We conclude:

Corollary 5.4 The norm $\left(P^{\cdot \cdot}\right)_{G / U}\left(N^{\cdot U}\right) \longrightarrow\left(I^{*}\right)^{G / U}\left(N^{\cdot U}\right)$ induces an isomorphism on total cohomology.

Since the total cohomology of the target is $H^{*}(G ; M)$, it follows that there is a spectral sequence $E_{2}^{p, q}=H_{-p}\left(G / U ; H^{q}(U ; M)\right) \Rightarrow H^{*}(G ; M)$. Reindexing by setting $s=-p$ and $t=d-q$ yields a first quadrant homology spectral sequence - the Tate spectral sequence:

$$
E_{s, t}^{2}=H_{s}\left(G / U ; H^{d-t}(U ; M)\right) \Rightarrow H^{d-s-t}(G ; M)
$$

We will not make any use of this spectral sequence, however. We have stated Corollary 5.4 only to motivate its analogue for spectra. This analogue could of course be stated purely in terms of profinite groups, but for future reference we record it in terms of Galois extensions.

Theorem 5.5 Suppose $E$ is a Galois extension of $F$ and $X$ a presheaf of spectra on $(E / F)$ with $c d(E / F ; X)<\infty$. Then for any finite Galois subextension $L / F$ with group $G$, the hypernorm

$$
N_{h}: \mathbb{H} .\left(G ; \mathbb{H}^{\cdot}(E / L ; X)\right) \longrightarrow \mathbb{H}^{\cdot}\left(G ; \mathbb{H}^{\cdot}(E / L ; X)\right)
$$

is a weak equivalence.

Here $c d(E / F ; X) \leq d$ if $c d\left(G(E / F) ; \tilde{\pi}_{q} X\right) \leq d$ for all $q$, in the sense described above.

Thomason's original proof is an induction on $c d(E / F ; X)$ that proceeds as follows. If this dimension is zero, then $\pi_{q}\left(\mathbb{H}^{\cdot}(E / L ; X)=H^{0}\left(E / L ; \pi_{q} X_{E}\right)\right.$. Hence $N_{h}$ is a weak equivalence by Tate's lemma combined with Lemma 5.2. 
At the inductive step, form the cofibre sequence $X \rightarrow T X \rightarrow C X$, where TX is the Thomason-Godement construction as usual and CX is the cofibre. Then $c d T X=0$ and $c d C X<c d X$; the result follows easily from this. See [22],7.1, for a proof using Postnikov towers.

\subsection{Transfer and hypertransfer}

Let $\mathrm{L}$ be a finite field extension of $\mathrm{F}$. In addition to the map $i: K F \longrightarrow K L$ arising from extension of scalars, there is a transfer map $t: K L \longrightarrow K F$ arising from restriction of scalars. We recall that the transfer has the following basic properties:

- Index formula. The composite $K F \stackrel{i}{\longrightarrow} K L \stackrel{t}{\longrightarrow} K F$ is multiplication by the index $[L: F]$

- Invariance under base change. The transfer commutes with base change - that is, given an arbitrary field extension $F^{\prime} / F$ there is a commutative diagram in the stable category

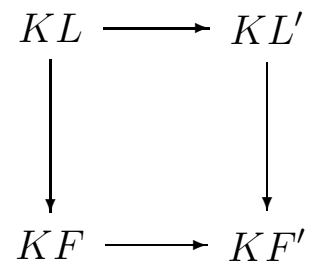

where $L^{\prime}=L \otimes_{F} F^{\prime}$ and the vertical maps are transfers (of course $L^{\prime}$ may not be a field, but the transfer is defined more generally).

- Norm formula. If L is Galois over F with group $\mathrm{G}$, then the composite $K L \stackrel{t}{\longrightarrow} K F \stackrel{i}{\longrightarrow} K L$ is the norm.

- Linearity. The transfer is a map of KF-module spectra.

We note that if $\mathrm{M} / \mathrm{F}$ is an arbitrary Galois extension with trivial pro- $\ell$ Sylow subgroup, it follows from the index and norm formulas that $(K F)_{(\ell)} \cong$ $\left((K M)_{(\ell)}\right)^{G(M / F)}$ and therefore the K-theory presheaf, localized at $\ell$, satisfies 
descent on M/F. In particular this suffices to prove the Lichtenbaum-Quillen conjectures for fields $\mathrm{F}$ with $c d_{\ell} F=0$, since a profinite group $\mathrm{G}$ satisfies $c d_{\ell} G=0$ if and only if the pro- $\ell$-Sylow subgroup of $\mathrm{G}$ is trivial.

Now consider the transfer $t: K L \longrightarrow K F$ in the case of a finite Galois extension. Then $t$ is not $\mathrm{G}$-equivariant with respect to the trivial action on $\mathrm{KF}$, because the forgetful functor $L-\bmod \rightarrow F-\bmod$ is only G-equivariant up to natural isomorphism. Here we are using the standard G-action in which $g \in G$ acts by tensoring up along $L \stackrel{g}{\longrightarrow} L$. Thus the transfer is only equivariant up to homotopy. In fact, however, $t$ is equivariant up to an infinity of higher homotopies, in the sense that $t$ extends canonically to a map

$$
t_{h}: \mathbb{H} .(G ; K L) \longrightarrow K F
$$

called the hypertransfer. The easiest way to think of this is to replace the G-action described above by the obvious equivalent action in which $\mathrm{G}$ acts by leaving the underlying F-vector space strictly alone and twisting the scalar multiplication. Then the transfer is strictly equivariant and we can define $t_{h}$ as the composite

$$
\mathbb{H} .(G ; K L)=E G_{+} \wedge_{G} K L \longrightarrow K L / G \longrightarrow K F
$$

At the level of plus constructions, the hypertransfer boils down to inclusion of the semi-direct product of $G L_{n} L$ and $G$ into $G L_{d n} F$, where $d=[L: F]$. For a rigorous account of all this, see [42],Example 2.30, and [22]. We note that a dual discussion applies to extension of scalars $i: K F \longrightarrow K L$, so that the augmentation $K F \longrightarrow \mathbb{H}^{\text {' }}(G ; K L)$ could just as well be called "hyperextension" $i_{h}$.

The hypertransfer has the following basic properties, each of which will be used at a crucial point in the proof of the main theorem below.

- Invariance under base change. If $F^{\prime}$ is an arbitrary field extension of $\mathrm{F}$ and $L^{\prime}=L \otimes_{F} F^{\prime}$, then the following diagram commutes in the stable category: 


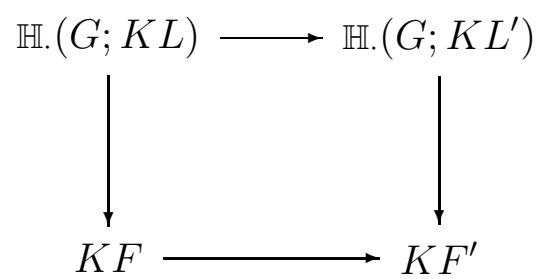

Here the vertical maps are the hypertransfers; in the application $L^{\prime}$ will in fact be a field, but the hypertransfer could have been defined more generally.

- Norm formula. The composite

$$
\mathbb{H} .(G ; K L) \stackrel{t_{h}}{\longrightarrow} K F \stackrel{i_{h}}{\longrightarrow} \mathbb{H}^{\cdot}(G ; K L)
$$

is the hypernorm.

- Linearity. The hypertransfer is a map of KF-module spectra (with respect to the obvious module structure on $\mathbb{H} .(G ; K L))$.

\subsection{Outline of the proof}

The proof is based on the two key lemmas 5.5 and 5.6, whose proofs are discussed in the final subsection. A Bott inductor for a finite Galois extension $\mathrm{L} / \mathrm{E}$ is an element $\tilde{\beta} \in \pi_{2} \mathbb{H}$. $(G ; k L)$ such that $t_{h}(\tilde{\beta})=\beta$. An arbitrary Galois extension $\mathrm{M} / \mathrm{E}$ admits Bott inductors if for every finite Galois subextension a Bott inductor exists.

Lemma 5.6 Let $M / E$ be a Galois extension such that either (a) $M=E_{\text {sep }}$ and $c d_{\ell} E \leq 1$; or (b) $M=E_{\infty}$ and $c d_{\ell} E_{\infty} \leq 1$. Then $M / E$ admits Bott inductors.

Lemma 5.7 Let $M / E$ be a Galois extension such that $c d_{\ell}(M / E)<\infty$ and $M / E$ admits Bott inductors. Then $\beta^{-1} k$ satisfies descent on $M / E$ :

$$
\beta^{-1} k E \stackrel{\cong}{\longrightarrow} \beta^{-1} \mathbb{H} \cdot(M / E ; k)
$$


We also need one more general fact about hypercohomology. Consider a sequence of Galois extensions $F \subset M \subset N$, and a presheaf of spectra $\mathrm{X}$ on $N / F$. Then there is a weak equivalence

$$
\mathbb{H}^{\cdot}(N / F ; X) \stackrel{\cong}{\longrightarrow} \mathbb{H}^{\cdot}\left(M / F ; \mathbb{H}^{\cdot}(N /-; X)\right)
$$

This can be seen in several ways. The simplest is to observe that the coefficient presheaf on the right is the direct image of $\mathbb{H}^{\cdot}(N /-; X)$ under the site morphism $G(N / F)_{f s e t s} \longrightarrow G(M / F)_{\text {fsets }}$; then one can use the fact that direct image functors preserve globally fibrant presheaves (Proposition 3.14). It is also easily proved directly in terms of homotopy fixed point sets (Section 1). Using this we obtain:

Lemma 5.8 Suppose $M / F$ has a filtration $F=M_{0} \subset M_{1} \subset \ldots \subset M_{n}$ with each layer $M_{i+1} / M_{i}$ Galois and $c d_{\ell} M_{i+1} / M_{i}<\infty$, and suppose $X$ is a presheaf of spectra on $M_{n} / F$ satisfying

$$
X_{M_{k}} \stackrel{\cong}{\longrightarrow} \mathbb{H}^{\cdot}\left(M_{k+1} / M_{k} ; X\right)
$$

for all $k$. Then $X \stackrel{\cong}{\longrightarrow} \mathbb{H}^{\cdot}(F ; X)$.

Proof: We proceed by descending induction over the filtration. At the inductive step, there is a commutative diagram

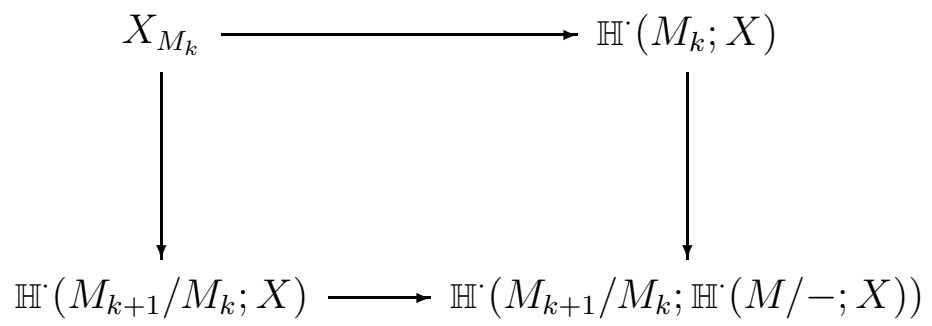

The lefthand map is a weak equivalence by assumption, and the righthand map is a weak equivalence by the remarks above. The bottom map is a weak equivalence because by inductive hypothesis the map on stalks is a weak equivalence.

Now suppose $\mathrm{F}$ is an $\ell$-good field containing $\ell$-th roots of unity. We suppose that $\mathrm{F}$ has finite transcendence degree over a field $F_{0}$ with $c d_{\ell} F_{0}=1$; 
the proof in the other case is quite similar. Replacing $F_{0}$ by its algebraic closure in $\mathrm{F}$ if necessary, we can assume $\mathrm{F}$ is purely transcendental over $F_{0}$. Furthermore we can assume $\mathrm{F}$ is is a separable algebraic extension of $E=F_{0}\left(t_{1}, \ldots, t_{n}\right)$ for some $n$ (since both $\bmod \ell$ K-theory and étale hypercohomology are invariant under purely inseparable extension), and moreover that $\mathrm{F}$ is finite over $\mathrm{E}$ (by a direct limit argument). In other words, we can assume $\mathrm{F}$ is a "function field" of dimension $n$ over $F_{0}$.

Now any such function field admits a "Tate-Tsen filtration" - that is, a filtration of $F_{\text {sep }} / F$ of the form $F=M_{0} \subset M_{1} \subset \ldots \subset M_{n}$ in which each layer $M_{i+1} / M_{i}$ is induced from an extension of the form $N_{\text {sep }} / N$ with $c d_{\ell} N \leq 1$. For example, if $F=F_{0}(t)$ we can take the filtration $F_{0}(t) \subset\left(F_{0}\right)_{\text {sep }}(t) \subset F_{\text {sep }}$, in which the first layer is induced from $F_{0} \subset\left(F_{0}\right)_{\text {sep }}$ and the second layer has $c d_{\ell} \leq 1$ by Tsen's theorem. A similar but somewhat more complicated argument constructs the filtration in the general case.

The extensions $N_{\text {sep }} / N$ admit Bott inductors by Lemma 5.6. Since the hypertransfer commutes with base change, each layer $M_{i+1} / M_{i}$ therefore ad-

mits Bott inductors. Hence $X_{M_{k}} \stackrel{\cong}{\longrightarrow} \mathbb{H}^{\cdot}\left(M_{k+1} / M_{k} ; X\right)$ by Lemma 5.7. Then Lemma 5.8 completes the proof.

\subsection{The key lemmas}

Suppose $c d_{\ell} F \leq 2$, and consider the map $\eta: k F \longrightarrow \mathbb{H}^{\cdot}(F ; k)$ in low degrees. One can show:

- There is a short exact sequence

$$
0 \longrightarrow H^{2}(F ; \mathbb{Z} / \ell) \longrightarrow \pi_{0} \mathbb{H}^{\cdot}(F ; k) \longrightarrow \mathbb{Z} / \ell \longrightarrow 0
$$

and $\mathbb{Z} / \ell=\pi_{0} k F \longrightarrow \pi_{0} \mathbb{H}^{\cdot}(F ; k)$ maps onto the quotient term $\mathbb{Z} / \ell$. Since $\mathrm{F}$ contains the $\ell$-th roots of unity, the norm residue symbol gives an isomorphism from $H^{2}(F ; \mathbb{Z} / \ell)$ to the $\ell$-torsion in the Brauer group of F.

- $\pi_{1} \mathbb{H}^{\cdot}(F ; k)=H^{1}(F ; \mathbb{Z} / \ell)=F^{\times} / \ell$ by Hilbert's Theorem 90 , and $\pi_{1}(\eta)$ is an isomorphism.

- Multiplication by $\beta$ is an isomorphism $\pi_{0} \mathbb{H}^{\cdot}(F ; k) \stackrel{\cong}{\longrightarrow} \pi_{2} \mathbb{H}^{\cdot}(F ; k)$. By the Mercurjev-Suslin theorem [28], $\pi_{2}(\eta)$ is an isomorphism. 
- $\pi_{n} k F=0$ for $n<0$, but $\pi_{-1} \mathbb{H}^{\cdot}(F ; k) \cong \pi_{1} \mathbb{H}^{\cdot}(F ; k)$ and $\pi_{-2} \mathbb{H}^{\cdot}(F ; k) \cong$ $\pi_{0} \mathbb{H}^{\cdot}(F ; k)$.

Now consider the commutative diagram in the stable category

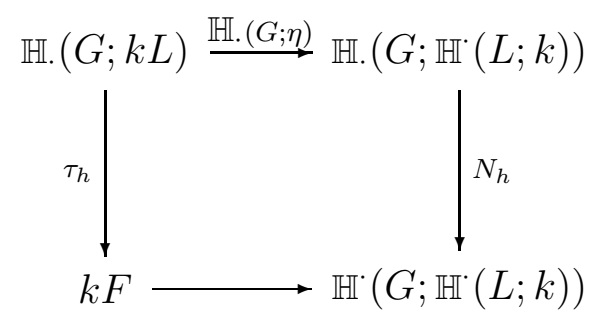

The bottom map is the composite

$$
k F \longrightarrow \mathbb{H}^{\cdot}(G ; k L) \stackrel{\cong}{\longrightarrow} \mathbb{H}^{\cdot}\left(G ; \mathbb{H}^{\cdot}(L ; k)\right)
$$

One might hope to prove Lemma 5.6 as follows. If we pretend for a moment that $\pi_{*}(\eta)$ is an isomorphism through degree 2 , then the same is true for $\mathbb{H} .(G ; \eta)$, using the hyperhomology spectral sequence. Since $N_{h}$ is a weak equivalence by the spectrum-level Tate theorem, it follows that $\pi_{2}\left(\tau_{h}\right)$ is an isomorphism. Hence we have a (unique) Bott inductor.

Unfortunately, as noted above, we do not have an isomorphism through degree 2 . Even when $c d_{\ell} F \leq 1$, the group $\pi_{-1} \mathbb{H}^{\cdot}(F ; k)$ gets in the way. In this case, however, the problem is easily circumvented by replacing the presheaf $k$ by its 0 -connected cover. The reader can then easily complete the proof of Lemma 5.6 when $c d_{\ell} F \leq 1$.

If $c d_{\ell} F=2$, then even the modified argument fails. The problem is with the Brauer group term in $\pi_{0} \mathbb{H}^{\cdot}(F ; k)$. This term can be eliminated by passing to more highly connected covers of $k$, but then we only succeed in eliminating the very group we are attempting to analyze! Nevertheless, there is a similar but much more delicate - argument that carries the day ([22], Theorem 7.16). One has to pick out just the right connected covers and Postnikov sections to isolate the Bott element. Of course, the Mercurjev-Suslin theorem again plays a crucial role.

We now turn to the proof of Lemma 5.7. We wish to show that $\eta$ : $k F \longrightarrow \mathbb{H}^{\cdot}(M / F ; k)$ induces an isomorphism on $\beta^{-1} \pi_{*}$. Let us first show that it induces an injection. Let $\mathrm{L} / \mathrm{F}$ be a finite Galois subextension with group 
G. Fix a Postnikov section $P^{n} k$ of the presheaf $k$. Note that $P^{n} k$ is a presheaf of $k F$-module spectra.

Lemma 5.9 There is a map $\phi: \mathbb{H}^{\cdot}\left(G ; P^{n} k\right) \longrightarrow \Omega^{2} P^{n} k F$ such that the composite

$$
P^{n} k F \longrightarrow \mathbb{H}^{\cdot}\left(G ; P^{n} k\right) \stackrel{\phi}{\longrightarrow} \Omega^{2} P^{n} k F
$$

is homotopic to multiplication by $\beta$.

In fact the lemma holds with $P^{n} k$ replaced by $k$ itself. The point of passing to a Postnikov section is that then

$$
\left.\operatorname{colim}_{L} \mathbb{H}^{\cdot}\left(L / F ; P^{n} k\right) \cong \mathbb{H}^{\cdot}(M / F) ; P^{n} k\right)
$$

by Example 3.22. To show that $\beta^{-1} \eta$ is injective, we will show that if $a \in \pi_{*} k$ and $\eta_{*} a=0$, then $\beta a=0$. For this purpose we may replace $k$ by a Postnikov section $P^{n} k, n>>0$, and then it follows immediately from the lemma that $\beta a=0$.

To construct $\phi$, consider the composition $\theta$ of multiplication and the transfer:

$$
k L \wedge P^{n} k L \longrightarrow P^{n} k L \longrightarrow P^{n} k F
$$

Taking adjoints yields a G-equivariant map $P^{n} k L \longrightarrow \mathcal{F}\left(k L, P^{n} k F\right)$, where $\mathcal{F}(-,-)$ denotes the function spectrum. We then define $\phi$ to be the composite

$$
\mathbb{H}^{\cdot}\left(G ; P^{n} k L\right) \longrightarrow \mathbb{H}^{\cdot}\left(G ; \mathcal{F}\left(k L, P^{n} k F\right)\right) \cong \mathcal{F}\left(\mathbb{H} .(G ; k L), P^{n} k F\right) \stackrel{\tilde{\beta}^{*}}{\longrightarrow} \Omega^{2} P^{n} k F
$$

where the first map is induced by the coefficient map $\theta$, the second is the universal coefficient equivalence, and the last map is pre-composition with a Bott inductor $\tilde{\beta}$. Using linearity of the hypertransfer, it is not hard to show that the composite map of the lemma is multiplication by $\beta$.

The proof of surjectivity is similar. Essentially, one shows that the composite $\mathbb{H}^{*}\left(G ; P^{n} k\right) \longrightarrow \Omega^{2} P^{n} k F \longrightarrow \Omega^{2} \mathbb{H} \cdot\left(G ; P^{n} k\right)$ is also multiplication by $\beta$; it then follows that for all $b \in \pi_{*} \mathbb{H}^{\cdot}(M / F ; k), \beta b$ is in the image of $\eta$. 


\section{References}

[1] Artin,M., Grothendieck Topologies, Harvard University lecture notes, 1962.

[2] Artin,M., On the joins of Hensel rings, Advances in Math 7 (1971), 282-296.

[3] Artin,M, Grothendieck,A., and Verdier,J.-L., Théorie des topos et cohomologie étale des schemas (SGA 4), Lecture Notes in Math 269,270,305, Springer, Berlin-Heidelberg-New York, 1972-73.

[4] Boardman, J.M., Conditionally convergent spectral sequences, unpublished manuscript, 1981.

[5] Bousfield,A.K., and Friedlander,E., Homotopy theory of $\Gamma$-spaces, spectra, and bisimplicial sets, Springer Lecture Notes in Math. 658 (1978), 80-130.

[6] Bousfield,A.K., and Kan,D., Homotopy limits, completions and localizations, Springer Lecture Notes in Math. Vol. 304 (2nd corrected printing), Springer-Verlag, Berlin-Heidelberg-New York, 1987.

[7] Brown,K.S. and Gersten,S.M., Algebraic K-theory as generalized cohomology theory, Springer Lecture Notes in Math. 341 (1973), 266-292.

[8] Dwyer, W. and Friedlander, E., Algebraic and etale K-theory, Trans. Amer. Math. Soc. 292 (1985), 247-280.

[9] Dwyer,W., and Mitchell,S., On the K-theory spectrum of a ring of algebraic integers, preprint 1993.

[10] Dwyer,W., and Mitchell,S., On the K-theory spectrum of a smooth curve over a finite field, to appear in Topology.

[11] Dwyer,W., and Spalinski, Homotopy theories and model categories, in Handbook of Algebraic Topology, I.M. James, ed., Elsevier Science B.V., Amsterdam-New York, 1995.

[12] Eisenbud,D, Commutative algebra with a view toward algebraic geometry, Springer-Verlag, New York, 1995. 
[13] Gabber,O., K-theory of Henselian local rings and Henselian pairs, Contemporary Math. 126 (1992), 59-70.

[14] Gillet, H. and Thomason, R., The K-theory of strict hensel local rings and a theorem of Suslin, J. Pure and Applied Alg. 34 (1984), 241-254.

[15] Goerss,P., Homotopy fixed points for Galois groups, Contemporary Math. 181 (1995), 187-224.

[16] Grothendieck,A., and Dieudonné,J., Eléments de Geometrie Algébrique I (EGA 1), Springer, Berlin-Heidelberg-New York, $1971 .$.

[17] Grothendieck,A. Étude local des schémas et des morphismes de schémas (troisieme partie), IHES Pub. Math. 28, 1966.

[18] Hartshorne,R., Algebraic Geometry, Graduate Texts in Mathematics, Vol. 52, Springer-Verlag, New York-Heidelberg-Berlin, 1977.

[19] Jannsen,U., Continuous étale cohomology, Math. Ann. 280 (1988), 207245.

[20] Jardine,J.F., Simplicial presheaves, J. Pure Applied Algebra 47 (1987), $35-87$.

[21] Jardine,J.F., Stable homotopy theory of simplicial presheaves, Can. J. Math. 39 (1987), 733-747.

[22] Jardine,J.F., Generalized étale cohomology theories, book manuscript, to appear in the Progress in Math. series, Birkhauser.

[23] Johnstone,P.T., Topos theory, Academic Press, London-New York, 1977.

[24] Kahn, B., Bott elements in algebraic K-theory, preprint.

[25] Kato,K. and Seito,S., Global class field theory of arithmetic schemes, Contemporary Math. 55 (1986), 255-332.

[26] MacLane,S., Categories for the working mathematician, SpringerVerlag, New York, 1971.

[27] May,J.P., Simplicial Objects in Algebraic Topology, University of Chicago Press, Chicago, 1992 (c1967). 
[28] Mercurjev,A.S., and Suslin,A.A., K-cohomology of Severi-Brauer varieties and the norm residue homomorphism, Math. USSR Izvestia 21 (1983), 307-340.

[29] Milne,J.S., Etale Cohomology, Princeton University Press, Princeton, New Jersey, 1980.

[30] Mitchell,S.A., The Morava K-theory of algebraic K-theory spectra, J. Algebraic K-Theory 3 (1990), 607-626.

[31] Mitchell, S.A., On the Lichtenbaum-Quillen conjectures from a stable homotopy-theoretic viewpoint, in Algebraic Topology and Its Applications, MSRI Publications 27, pp. 163-240, Springer-Verlag, New York, 1994.

[32] Mitchell,S.A., Class groups and general linear group cohomology for rings of algebraic integers, to appear in Math. Zeit.

[33] Mitchell,S.A., Units and general linear group cohomology for rings of algebraic integers, to appear in Math. Zeit.

[34] Nisnevich, Y.A., The completely decomposed topology on schemes and associated descent spectral sequences in algebraic K-theory, Algebraic K-theory: Connections with Geometry and Topology , NATO ASI Series C, vol. 279, Kluwer, Dordrecht, 1989, pp. 241-342.

[35] Quillen, D., Higher Algebraic K-theory 1, Springer Lecture Notes in Math. 341 (1973), 85-147.

[36] Quillen, D., Homotopical algebra, Lecture Notes in Math. 43 (Springer, Berlin, 1967).

[37] Serre,J.P., Cohomologie Galoisienne, Lecture Notes in Math. 5, Springer-Verlag, 1973.

[38] Suslin,A., On the K-theory of algebraically closed fields, Invent. Math. 73 (1983), 241-245.

[39] Suslin,A., On the K-theory of local fields, J. Pure Appl. Alg. 34 (1984), 301- 318. 
[40] Suslin,A., and Voevodsky,V., Bloch-Kato conjecture and motivic cohomology with finite coefficients, preprint 1995.

[41] Tamme,G., Introduction to Étale Cohomology, Springer-Verlag, New York-Heidelberg-Berlin, 1994.

[42] Thomason,R., Algebraic K-theory and etale cohomology, Ann. Scient. Ecole Norm. Sup. 13 (1985), 437-552.

[43] Thomason,R., Survey of algebraic vs. etale topological K-theory, Contemporary Math. 83 (1989), 393-444.

[44] Thomason,R. and Trobaugh,T. Higher algebraic K-theory of schemes and of derived categories, The Grothendieck Festschrift, Volume III, Progress in Mathematics v. 88, Birkhauser 1990, pp. 247-436.

[45] Waterhouse, W., Profinite groups are Galois groups, Proc. Amer. Math. Soc. 42 (1973), 639-640.

[46] Wiles, A., The Iwasawa conjecture for totally real fields, Annals of Math. 131 (1990), 493-540. 